\title{
1 Absolute Scaling of Single-Cell Transcriptomes Reveals Pervasive \\ 2 Hypertranscription in Adult Stem and Progenitor Cells
}

Yun-Kyo Kim ${ }^{1,2,3^{*}}$, Miguel Ramalho-Santos ${ }^{2,3^{*}}$

${ }^{1}$ Program in Developmental and Stem Cell Biology, Hospital for Sick Children, Toronto ON M5G 0A4, Canada

2Lunenfeld-Tanenbaum Research Institute, Mount Sinai Hospital, Toronto ON M5T 3L9, Canada

11 Canada

12 "Correspondence:

13 Miguel Ramalho-Santos, mrsantos@lunenfeld.ca

14 Yun-Kyo Kim, yun-kyo.kim@sickkids.ca

Key Words: single-cell RNA-seq; hypertranscription; ERCC; UMI; absolute scaling, stem cells; progenitor cells; regeneration

Word Count: Abstract $=269$; Text (excluding title page, abstract, references, methods) $=$ 5612

\section{SUMMARY STATEMENT}

25 of transcription across adult cell lineages 
ABSTRACT

27 Hypertranscription facilitates biosynthetically demanding cellular state transitions

28 through global upregulation of the nascent transcriptome. Despite its potential widespread relevance, documented examples of hypertranscription remain few and limited predominantly to early development. This limitation is in large part due to the fact that modern sequencing approaches, including single-cell RNA sequencing (scRNAseq), generally assume similar levels of transcriptional output per cell. Here, we use molecule counting and spike-in normalization to develop absolute scaling of single-cell RNA sequencing data. Absolute scaling enables an estimation of total transcript abundances per cell, which we validate in embryonic stem cell (ESC) and germline data and apply to adult mouse organs at steady-state or during regeneration. The results reveal a remarkable dynamic range in transcriptional output among adult cell types. We find that many different multipotent stem and progenitor cell populations are in a state of hypertranscription, including in the hematopoietic system, intestine and skin. Hypertranscription marks cells with multilineage potential in adult organs, is redeployed in conditions of tissue injury, and can precede by 1-2 days bursts of proliferation during regeneration. In addition to the association between hypertranscription and the stem/progenitor cell state, we dissect the relationship between transcriptional output and cell cycle, ploidy and secretory behavior. Our analyses reveal a common set of molecular pathways associated with hypertranscription across adult organs, including chromatin remodeling, DNA repair, ribosome biogenesis and translation. Our findings introduce an approach towards maximizing single-cell RNA-seq profiling. By applying this methodology across a diverse collection of cell states and contexts, we put forth

49 hypertranscription as a general and dynamic cellular program that is pervasively

50 employed during development, organ maintenance and regeneration. 


\section{INTRODUCTION}

52 Cells dynamically regulate their biosynthetic capacities to fulfill the requirements of 53 demanding cell state transitions ${ }^{1}$. At the level of transcription, cells can meet these demands by entering a state of relative hypertranscription, which is characterized by a global upregulation of nascent transcriptional output ${ }^{2}$. This global shift powers biological phenomena requiring substantial increases in total biomass, such as rapid proliferation, secretion, and cell activation ${ }^{1,2}$. Hence, hypertranscription has been proposed to play major roles in developmental transitions, adult organ homeostasis, and tumorigenesis ${ }^{2}$. Our group and others have reported evidence supporting the occurrence and functional relevance of hypertranscription in mouse embryogenesis and select human cancer cell types $^{3-8}$. However, the occurrence of hypertranscription in adult physiology remains largely unexplored

63

Despite the paucity of data in adult tissues, work in mouse embryos and mESCs has identified five recurring "hallmarks" of hypertranscription. First, hypertranscribing cells display a transcriptome-wide increase in gene expression, including at housekeeping genes and ribosomal RNA (rRNA) ${ }^{4,9}$. These changes occur at the level of both steadystate and nascent transcripts, and result in measurable increases in total cellular RNA content ${ }^{4}$. Second, hypertranscription is dependent on a decondensed and distinctly permissive chromatin landscape, maintained by the activity of euchromatic remodeling factors ${ }^{10,11}$. One notable factor is the ATP-dependent remodeler Chd1, which binds specifically to $\mathrm{H} 3 \mathrm{~K} 4 \mathrm{me} 3$ and is required for the transcriptional output of proliferating epiblast cells ${ }^{4}$. Third, hypertranscribing cells upregulate protein synthesis/translational machinery, which is necessary for the continuous translation and steady-state maintenance of Chd1 and several other unstable euchromatic regulators ${ }^{10}$. Fourth, hypertranscribing cells endogenously accumulate promoter-proximal double strand breaks (DSBs), thus displaying a heightened dependency on DNA repair factors ${ }^{12}$. Fifth, hypertranscription is thought to be mediated by the expression of general and "universally amplifying" transactivators, of which the Myc family of transcription factors is the best characterized ${ }^{3,13}$.

A critical requirement for detecting hypertranscription is the ability to distinguish absolute differences in transcript expression between samples or cell states. Despite substantial modern advancements in bulk and single-cell transcriptomic profiling, such measurement is complicated by the standard use of between-transcriptome normalization procedures ${ }^{14}$. For instance, normalization to read-depth in RNA-seq data or housekeeping genes in qRT-PCR assume similar amounts of cellular RNA content between samples of interest. Analysis of scRNA-seq commonly employs similar global scaling approaches, which are used to minimize the impact of stochastic technical effects including capture inefficiencies, amplification biases, and variable sequencing depths ${ }^{14-16}$. However, the global scaling tools used to eliminate these technical biases

92

93 also suppress genuine biological differences in mRNA content, thereby masking the detection of global shifts driven by hypertranscriptive states ${ }^{14}$. Thus, the analysis of 
94 hypertranscription at single-cell resolution requires development of alternative

95 methodologies.

96

97 In previous work investigating hypertranscription, we circumvented the problem of

98 between-sample normalization by developing cell-number-normalized (CNN) profiling

99 methods which accurately reproduce absolute cellular transcript levels ${ }^{3,4,9}$. Here, we

100 apply an analogous approach to single-cell transcriptomic data by leveraging unique

101 molecular identifiers (UMIs) or External RNA Controls Consortium (ERCCs) spike-in

102 sequences, two tools commonly included in various scRNA-seq protocols ${ }^{17-19}$. We use

103 these tools to perform absolute scaling of scRNA-seq data, which we validate as being

104 able to faithfully capture hypertranscription in ground-truth settings and developmental

105 cases of hypertranscription previously demonstrated using bulk CNN RNA-seq. We

106 apply this methodology to multiple scRNA-seq datasets in heterogenous adult tissues

107 and report the identification of progenitor cell lineages displaying hallmarks of

108 hypertranscription during homeostasis and regeneration. These results support a model

109 where hypertranscription acts as a general mechanism to facilitate dynamic regulation

110 of cellular biosynthetic capacity during development, organ homeostasis, and

111 regeneration. 


\section{RESULTS}

113 Absolute Scaling Accurately Estimates Transcript Content in Ground Truth Data To estimate absolute cellular transcript abundances in scRNA-seq data, we focused on protocols using UMIs or ERCCs for normalization and quality control (Supp Fig 1A). ERCC spike-in sequences are typically used in flow cytometric methods (e.g., Smartseq2, CEL-seq) and are added to lysis buffer solutions at known concentrations. Similar to our previous bulk CNN RNA-seq methodology, the equal distribution of spike-ins prior to capture allows scaling factors to be generated using the ERCC fraction of reads ${ }^{3}$. These scaling factors normalize for downstream technical effects while also retaining biological differences in total transcript abundance ${ }^{14}$. In contrast, UMls are typically used in droplet and split-pooling methods (10X Genomics, sci-RNA-seq3) and uniquely label each transcript molecule during mRNA capture. Although raw UMI counts retain variation due to per-cell capture efficiency and dropout, we reasoned that they can still provide an acceptable estimate of transcript abundance when analyzed across large enough samples of cells. Collectively, we refer to these approaches to normalization as absolute scaling, in contrast to the global scaling performed in typical normalization of scRNA-seq data (Supp Fig 1B).

To evaluate whether UMls and ERCCs can be used for absolute scaling, we explored two ground truth scenarios. Firstly, we assessed whether using raw UMI counts can distinguish differences in total transcript abundance from UMI-containing libraries generated from singlets vs doublets of cells. As doublet libraries are expected to contain roughly twice the mRNA of singlets, they represent one scenario of hypertranscription with mostly uniform amplification across the transcriptome. We used three publicly available 10X Genomics datasets with experimental doublet annotation: in two datasets based on peripheral blood mononuclear cells (PBMCs), we found that doublets contained roughly 1.8 to 1.9 times the total transcripts as singlets, consistent with previous reports (Supp Fig 2A) ${ }^{20,21}$. These doublets also displayed similar increases in the expression of ribosomal and housekeeping genes (Supp Fig 2B). In mixtures of different cell types, such as human and mouse, transcript content of singlets was reflective of known cell size differences (17946.0 UMls in 293T vs 10522.0 UMIs in $3 T 3)^{22}$. Importantly, transcripts in doublets were consistent with aggregation of these two cell types (mean of $26222.5 \mathrm{UMIs}$ ), indicating accurate capture of total abundance

\section{(Supp Fig 2A).}

Secondly, we assessed whether raw UMls and ERCCs can recapitulate mRNA differences in artificially generated pseudo-cell libraries. We took advantage of the previously published "scRNA-seq Mixology" experiment, which generated UMI and ERCC-containing CEL/Sort-seq libraries using extracted mRNA over a 4-step dilution series $^{23}$. This series contained material from three different human cell lines and spanned an order of magnitude, sufficiently representing the variation found in hypertranscribing cells ${ }^{3,4}$. As the mRNA content of each single-cell library was known, we evaluated the reproduction of transcript abundance using either raw total UMIs or 
ERCC-derived per-cell size factors. Compared to global scaling, absolute scaling using either method more accurately reproduced ground truth differences in pseudo-cell transcript levels. (Supp Fig 3A, C). Importantly, changes in transcript abundances were seen to be largely uniform across detected genes, analogous to what would be observed in biological hypertranscription (Supp Fig 3B, D). We found that regardless of platform, ERCC normalization slightly outperformed raw UMI counts and generated lower inter-cell variability in transcript abundances, particularly at higher ground truth RNA amounts (Supp Fig 3A-D, E-F). Together, these results document the utility of ERCCs and UMls in absolute scaling and recovering global transcriptomic differences in scRNA-seq data.

\section{Validation of Absolute Scaling Using Embryonic Hypertranscription Data} We next sought to test absolute scaling in previously established contexts of hypertranscription. The early mouse embryo is especially well characterized in this regard and can be modeled in-vitro using $\mathrm{mESCs}^{24}$. Specifically, serum/LIF-grown mESCs are hypertranscriptional and represent the rapidly proliferating early postimplantation epiblast, while mESCs under dual GSK/MEK inhibition (2i media) are comparatively hypotranscriptional and represent a pre-implantation-like state ${ }^{9,24}$. We performed absolute scaling on an ERCC-spiked mESC dataset generated using Fluidigm $\mathrm{C} 1$ containing both serum and $2 \mathrm{i}$ cells ${ }^{25}$. While globally-scaled data showed only a modest 1.02 -fold change between the two cell states, ERCC-normalization revealed that serum mESCs contain 2.37-fold higher transcript counts than $2 \mathrm{i}$ mESCs, in concordance with findings from bulk CNN RNA-seq data (Fig 1A-F) ${ }^{9}$.

To confirm whether this increase in total transcript abundance is reflective of hypertranscription, we turned to evaluating known hallmarks. We found that under absolute scaling, mESCs display global transcriptomic upregulation across both highly and lowly expressed genes (Fig 1G-H). These upregulated genes include chromatin remodelers, DNA repair factors, ribosomal genes, housekeeping genes, and Myc (Supp Fig 4 A-B). Performing Gene Ontology (GO) analysis using differentially expressed genes in serum vs $2 \mathrm{i}$ mESCs extracted from globally scaled data revealed an enrichment for terms associated predominantly with metabolic pathways, mainly highlighting the metabolic differences between the cell states. (Fig 1I). In contrast, GO analysis starting with absolute scaling revealed a high enrichment of terms related to translational/ribosomal processes and biosynthesis (Fig $1 \mathrm{~J}$ ). These findings are in strong agreement with our previous bulk-CNN characterizations of hypertranscribing serum vs $2 \mathrm{i} \mathrm{mESCs}{ }^{9}$.

We then looked to validate our absolute scaling methodology using data from cells in vivo, rather than cultured cells. We have previously shown that during mouse embryogenesis, the mid-gestation primordial germ cell (PGC) lineage undergoes Mycdriven hypertranscription relative to the surrounding soma ${ }^{3}$. To assess whether hypertranscription can also be captured during this period in scRNA-seq data, we used 
a 10X Genomics dataset generated from whole mouse gonads at seven timepoints between E11.5 to P5 ${ }^{26}$. When identified by expression of Oct4, globally scaled PGCs appear as having minimal difference in total transcript counts compared to soma (Fig1 $\mathbf{K}, \mathbf{M}, \mathbf{O}$ ). In contrast, an analysis of raw UMls revealed that PGC transcript abundances are 1.62-fold and 2.17-fold increased over soma at E12.5 and E14.5, respectively (Fig $1 \mathrm{~L}, \mathrm{~N}, \mathrm{P})$. These elevations in UMI counts correspond precisely to the timepoints of PGC mitotic expansion during development and are accompanied by increases in G2/M and S-phase cell cycle scores (Fig 1Q) ${ }^{26,27}$.

Similar to serum mESCs, the transcriptomes of E12.5 and E14.5 PGCs normalized by absolute scaling display a high overrepresentation of genes related to hypertranscription hallmarks (Supp Fig 4C-D). Importantly, these differential expression changes are largely absent prior to and following the burst in PGC proliferation, in agreement with the restriction of hypertranscription to mid-gestation development ${ }^{3}$. Together, these data indicate that absolute scaling, whether based on UMI or ERCC-spiked data, accurately

213

214

215 reproduces embryonic hypertranscription in scRNA-seq data both in culture and in vivo, prompting us to apply it to complex adult cell datasets.

\section{Transcriptional Content Heterogeneity Across Adult Cell Lineages}

We previously hypothesized that the rapid proliferation required for turnover in select organs systems may be facilitated in part by hypertranscription ${ }^{2}$. We looked to explore this possibility by applying the absolute scaling methodology described above to existing single-cell atlases of mouse organs. Large-scale scRNA-seq studies have begun to assemble compendia of cellular diversity, which serve as general resources of organism-wide cell expression profiles ${ }^{28-30}$. We chose to focus on the Tabula Muris

223

224

225

226

227

228

229

230

231

232

233

234

235

236

237

238

239 atlas of twenty mouse organs, which was particularly amenable to absolute scaling due to its inclusion of both ERCC spike-ins and UMls ${ }^{31}$. Within the atlas, these inclusions are divided between datasets generated using Smart-seq2 (FACS) and 10X Genomics (Droplet) platforms, respectively. In the following analysis, we focused primarily on FACS datasets due to the wider organ selection and greater read-depth, as well as the better performance of ERCC normalization for absolute scaling (see Supp Fig 3) ${ }^{14}$. Where relevant, we corroborated significant findings using data based on raw UMI counts.

To apply absolute scaling to the Tabula Muris, we first ensured linear correlations of ERCC expression between organ datasets, as well as validated that individual ERCC species were present at expected frequencies (Supp Fig 5G,I). In addition, we performed filtering for ERCC levels, library size, and doublets for all datasets (Supp Fig $5 \mathrm{H}$, and see Methods) $)^{32}$. These steps resulted in a total of 42,047 ERCC-containing and 45568 UMI-containing libraries, which upon absolute scaling showed an interquartile range (IQR) of 1,119,112 transcripts and 6377 UMls, respectively (Supp Fig 5A-D). Where available, FACS and Droplet datasets of the same organ were correlated at the level of both gene expression and median transcripts per cell (Supp Fig 5J-K). 
Given the substantial IQR of cellular transcript content in both FACS and Droplet datasets, we next looked to assess whether transcript content differed significantly at the level of individual organ systems. Remarkably, we found that the Tabula Muris displays substantial variation in median transcript counts per organ, spanning a 6.63fold difference in FACS data and a 3.78-fold difference in 10X data (Fig 2A, Supp Fig 6A, Table S1). The distribution of transcript abundances within organs also varies substantially, with the highest variance in the large intestine (FACS IQR $=2,454,958$ ) or bladder (10X IQR $=10987)$ and lowest variance in the spleen (Smartseq 2 IQR $=199384,10 X$ IQR $=2174$ ) (Fig 2A, Supp Fig 6A-B, Table S1). Interestingly, we noticed that datasets derived from the hematopoietic, intestinal, and integumentary organs - systems classically associated with continuous turnover ${ }^{33}$ - rank highly amongst other organs in transcript content and are bimodally distributed (see below).

We next looked to visualize the distribution and heterogeneity of transcript abundance in the Tabula Muris. As absolute scaling would be expected to change the structure of our data (Fig 1 A vs B), we performed dimensionality reduction under global scaling, but visualized transcript counts generated under absolute scaling. We found that even under these conditions of RNA content parity, the distribution of transcript counts are heterogenous and a defining characteristic of many cell clusters (Supp Fig 7A-B). In addition, we observed widespread presence of transcript abundance gradients between highly separated groups of cells, suggesting intermediate states of cellular transcript content. To further explore these differences, we leveraged cell type annotations by the Tabula Muris Consortium, which revealed that transcript counts between different cell types span a remarkable 15.79-fold difference in FACS data (over 82 cell types) and a 8.29-fold difference in 10X data (over 56 cell types) (Fig 2B, Supp Fig 6B, Table S1) ${ }^{31}$. Interestingly, we found that a large proportion of the highest transcript-count cells are associated with an adult stem-cell or progenitor-cell identity, particularly from the bone marrow, intestine, and skin datasets. These findings are supported by the observation of higher levels of uridine incorporation in intestinal crypts, where stem and progenitor cells reside (ref ${ }^{34}$ and see below). In contrast, we found that cell-types with the lowest transcript counts are often associated with more highly differentiated or terminal cell states (Fig 2B, Supp Fig 6B, Table S1). Some notable exceptions to this association between elevated transcript counts and stem/progenitor cell states are discussed below. The accuracy of these differences was supported by transcript abundances in T-cells, B-cells, macrophages, and endothelial cells, which together display minimal variation while spanning multiple organ datasets (Supp Fig 8A-B).

Together, these data reveal a remarkable degree of inter- and intra-organ variation with regards to transcript content per cell. As a corollary, these findings suggest that

282 application of global-scaling approaches in complex tissues, while useful for identifying outlier modules of differential expression, are insufficient to capture important biological information with regards to transcriptional output per cell. 


\section{Relationship Between Elevated Transcriptional Output and the Cell Cycle} We next looked to evaluate whether high-content cell types were possible candidates for novel hypertranscribing populations. As hypertranscription is thought to facilitate rapid proliferation in embryonic contexts, we assessed the relationship between cell cycling and transcript abundance ${ }^{2}$. Using globally-scaled expression data, we performed cell cycle staging as previously described ${ }^{35}$ and evaluated the proportion of cycling and non-cycling cells within each annotated cell identity. Surprisingly, when identities were ranked by the proportion of non-cycling cells, we observed a roughly bimodal distribution with regards to total transcript content (Supp Fig 9, Supp Fig 10). Cell types canonically associated with rapid turnover, including progenitor cells of the bone marrow, skin, and intestines, display high proportions of G2/M and S-phase cells alongside elevated transcript counts. In contrast, some non-cycling G1 cells of the liver and bladder urothelium also display heightened transcript abundance.

We hypothesized that the properties of these latter cell types might in part be driven by alternative mechanisms, including somatic polyploidy. Polyploidy has been well documented in both hepatocytes and superficial urothelial cells, and arises through either incomplete cytokinesis or endoreplication ${ }^{36-41}$. Although we lacked DNA content information to directly measure these processes, we took advantage of previous studies identifying markers specific for polyploid cell populations. Using the Tabula Muris FACS liver dataset, we found that cell clusters with elevated transcript abundances are also enriched in markers of $4 \mathrm{n}$ hepatocyte Mixipl, Lifr, and $\mathrm{Nr}_{1} \mathrm{i}^{42}$ (Supp Fig 11A-D). Similarly, we found that high-transcript cells within the FACS bladder dataset coexpress Krt20 and Upk2 while lacking Trp63, a combination specific for 4n+4n superficial cells ${ }^{36}$ (Supp Fig 11E-O). Thus, these data suggest that estimation of mRNA abundance using absolute scaling is consistent with expected differences driven by polyploidy ${ }^{38}$.

A further exception to the general association between high transcript content and cycling stem/progenitor cells appears to be secretory terminally differentiated cells, such as goblet and Paneth cells within the intestinal epithelium (Supp Fig 14, see below). We had previously speculated that the biosynthetic demand of secretory cells may require features of hypertranscription ${ }^{2}$. This possibility is supported by the present analysis but warrants further investigation. Taken together, these analyses indicates that elevated transcriptional counts are generally associated with cycling stem/progenitor cells across multiple organs, with important exceptions observed in terminally differentiated cells that may be in part explained by polyploidy or high secretory output. using properties previously defined in bulk RNA-seq studies of embryonic cell 
populations (see Introduction and ref $^{2}$ ). First, we chose a global approach by evaluating the atlas-wide expression of various transcriptional signatures representative of relevant biological processes. At the level of single cells, we found that transcript abundance is robustly correlated to $\mathrm{GO}$ hallmark gene sets previously associated with hypertranscription, such as chromatin organization, RNA Pol II activity, ribosome biogenesis, DNA repair, and cell division (Fig 2C-E, Supp Fig 12A-C). We also found that a signature of embryonic hypertranscription, generated using the earlier Kolodziejczyk et al ${ }^{25}$ dataset, is highly correlated with transcript content in adult cells (Fig 2C). These associations are continuous, suggesting that cells do not endogenously rest at discrete levels of transcription. Rather, these data suggest that transcriptional output is fine-tuned across a wide spectrum by euchromatin regulation, RNA stability, translation, and other related processes.

We next sought to assess whether these hallmarks are reflected at the level of the expression of key individual regulators of hypertranscription. We found that the absolute-scaled expression of Chd1, transcription factors of the Myc family (see below), several DSB repair factors, mTOR, and ribosomal proteins are robustly correlated with single-cell transcript content (Fig 2F-G, Supp Fig 12D-I, Table S2). Additionally, performing enrichment analysis on the set of genes with correlation coefficients of $>=0.75$ to transcriptional content per cell revealed strong enrichments for GO terms related to translation, transcription, and metabolic processes (Fig $2 \mathrm{H}$, Table S3).

A key hypothesis underlying the mechanistic basis of hypertranscription is the activity of universally amplifying transcription factors, which bind and regulate large portions of the transcriptome. Experimental evidence for both Myc and Yap-driven hypertranscription have been reported in embryonic contexts ${ }^{3,7,8}$. To explore whether the role of these transcription factors is retained in adult cells, we first used ChIP Enrichment Analysis (ChEA) on the Tabula Muris using genes with a $>=0.75$ Spearman's coefficient to transcript abundance. ChEA performs gene-set enrichment using protein-DNA interactions derived from ChIP-seq and DamID studies ${ }^{43}$. Interestingly, we found that genes with strong correlations to transcript content are highly enriched in Myc and NMyc ChEA interactions across several biological contexts (Supp Fig 12J, Table S3). In contrast, correlated genes display weaker enrichment in Yap interactions (Table S3). These differences are reflected in the overall higher correlation of per-cell transcript content with Myc over Yap (Supp Fig 12G-I). Thus, the Myc family of transcription factors, more so than Yap, are strongly associated with hypertranscription in adult stem/progenitor cells. This analysis further reveals other transcription factors that have not to date been studied in the context of hypertranscription and display chromatin binding patterns strongly linked to transcript content in adult stem/progenitor cells (Supp Fig 12J, Table S3). These transcription factors, such as Eklf, E2f1, and Zfx, deserve to be revisited for potential roles in hypertranscription. 
Taken together, these data demonstrate a strong congruity of hypertranscription hallmarks between established embryonic models and stem/progenitor cells of adult tissues ${ }^{3-5,7-10,25}$. These similarities indicate that adult cycling stem/progenitor cells redeploy an embryonic hypertranscriptional program to meet the biosynthetic demands of organ renewal.

\section{Hypertranscription Marks Cells with Multilineage Potential in Adult Organs}

The presence of hypertranscription hallmarks within adult multipotent stem/progenitor cells led us next to probe the association between transcript content and lineage progression. We first asked whether cellular transcript counts are correlated with any previously established methods of inferring differentiation trajectories in scRNA-seq data. Differentiation progression has been captured using feature counts (number of expressed genes) or quantile polarization scores of principal components of the data (possibly reflecting polarized biological activity), providing the framework for the CytoTRACE and VECTOR trajectory inference tools, respectively ${ }^{44,45}$. Across individual cells of each organ dataset, we found that transcript abundance is well correlated with both metrics, displaying particularly strong associations with feature counts (Supp Fig 13A-B). These data suggest that transcriptional output, alongside transcript diversity, decreases along differentiation trajectories concomitant with a loss of multipotent capacity.

To explore this concept in the context of a well-defined differentiation model, we focused specifically on hematopoiesis within the bone marrow, the progression of which has been extensively characterized ${ }^{46}$. We found that ranking cell-types by absolute transcript abundance approximates the known hematopoietic hierarchy from multipotent stem cells to terminal differentiated mononuclear cell types (Fig 3A-C). Importantly, hematopoietic stem/progenitor cells display the previously explored hallmarks of hypertranscription, including upregulation of chromatin remodelers, DNA repair factors, ribosomal genes, and housekeeping genes (Fig 3D), as well as a substantial enrichment of the embryonic stem cell serum hypertranscription signature (Fig 3E). Moreover, we found that hematopoietic stem/progenitor cells display higher levels of both steady-state and nascent (intronic) reads, in agreement with the notion that hypertranscription is primarily regulated at the level of nascent transcription ${ }^{2}$ (Fig 3F).

Consistent with the model that hypertranscription acts in adult progenitors to facilitate renewal, we identified concordant results in stem cells of the skin and adult large intestine. In the interfollicular epidermis, Top2a+ cycling basal stem cells contain elevated transcript abundance compared to superficial epidermal cells (Supp Fig 14) ${ }^{31}$. In the intestine, multipotent $\mathrm{Lgr} 5+$ stem cells in crypt base display elevated steady-state and nascent transcripts compared to the more terminally differentiated enterocyte and brush cell populations (Supp Fig 15A-E). These stem cells, which rapidly replenish the colonic epithelium during homeostatic turnover, widely display the previously explored hallmarks of hypertranscription (Supp Fig 15F-H) ${ }^{47}$. Independent support for our 
412 findings is provided by Jao and Salic, who reported that intestinal crypts, where 413 stem/progenitor cells reside, have high levels of incorporation of EU, a marker of 414 nascent transcription ${ }^{34}$. Interestingly, we also observed a substantial enrichment of 415 transcripts in goblet cells, which are required for continuous mucin production (Supp

416 Fig 15D). The presence of high transcript counts in this population adds further support 417 to a novel role of hypertranscription in driving secretory cell output and is deserving of 418 further investigation.

\section{Dynamic Modulation of Hypertranscription During Adult Cell Differentiation}

We next looked to characterize dynamic changes in hypertranscription through the course of differentiation. We performed pseudotemporal ordering using Monocle on isolated hematopoietic cell trajectories representing the differentiation pathways of granulocytes, erythroblasts, and monocytes ${ }^{48}$. In granulocytes, we found that transcript abundance follows a gradual decrease throughout differentiation towards terminal identities (Fig 4A-C). To further probe transcriptional dynamics during this differentiation path, we used k-means clustering to organize differentially expressed genes into 6 classes (Fig 4D-I). Despite some overlap, these classes are enriched with unique biological processes, such as translational components in Class 1 and cell cycle genes in Class 5. Remarkably, we found that only Class 1 genes are downregulated substantially faster than the average total downregulation of transcriptional output over pseudotime, while all other classes track with the average or lag behind (colored lines in Fig 4D-I). These results suggest that in the context of differentiation, the exit from hypertranscription may be primarily initiated via the downregulation of ribosome biogenesis and translational output. The preferential protein instability of euchromatin and transcriptional activators that we have previously reported in embryonic stem cells may similarly in adult cells make hypertranscription dependent on high translational capacity ${ }^{10}$.

In the erythroblast and monocyte trajectories, we observed similar results with progressive losses of total transcript content with pseudotime (Supp Fig 16). In addition to this overall decline, we detected small populations of intermediate cells with transient increases in transcript content, reflected most strikingly in the levels of ribosomal and housekeeping genes (Supp Fig 16J, K). Interestingly, these brief bursts of increased transcription coincide with the onset of key differentiation effector genes that rapidly rise to very high levels, such as the induction of beta-2 hemoglobin in developing erythroblasts (Supp Fig 16N). It is possible that these transient rises in transcriptional output support high levels of synthesis needed for the rapid production of differentiation effector proteins. Importantly, while global scaling would correctly identify the onset of these differentiation markers, it would entirely miss the remarkable dynamics of global transcription during lineage commitment, including large scale shifts in the levels of the transcription and translation machineries (Supp Fig 160). Taken together, our analyses uncover a remarkably rich level of dynamics in global transcription at the single cell level 
454 and point to a pervasive redeployment of hypertranscription beyond embryogenesis in adult stem/progenitor cells.

\section{Rapid Induction of Hypertranscription During Adult Organ Regeneration}

460

The observation that hypertranscription is dynamically regulated during physiological renewal of adult organs led us to explore its status during regeneration. We first focused on the context of skeletal muscle repair, which is mediated by the rapid proliferation and differentiation of resident muscle satellite stem cells ${ }^{49}$. This context was of particular interest as steady-state satellite stem cells display relatively low transcript abundance in the Tabula Muris (see Fig 2, Table S1). Remarkably, we found that the total transcript content of satellite stem cells ranks highest compared to other cell types across a dataset of cardiotoxin-induced tibialis muscle injury ${ }^{50}$ (Fig 5A-C). When stratified into specific days post-injury (DPI), we found that non-injured and $21 \mathrm{DPI}$ satellite stem cells display comparably low transcript content (Fig 5D). In contrast, 0.5 DPI satellite stem cells display a notable 3.5 -fold upregulation of transcriptional output, which peaks at 2 DPI and slowly returns to baseline over the next 19 days. This rapid elevation of transcription upon injury precedes a large increase in the numbers of satellite stem cells, which rise by nearly two orders of magnitude across a 3-day time period (Fig 5D). In agreement with our data on hypertranscribing hematopoietic and epithelial stem/progenitor cells (Figs 2-4, Supp Fig 14-15), we found that the genes most differentially expressed in $0.5 \mathrm{DPI}$ vs non-injured satellite stem cells are enriched for functions in ribosome biogenesis, translation regulation and RNA processing (Fig 5E). Together, these data indicate that muscle satellite stem cells enter a state of injuryinduced hypertranscription that precedes rapid expansion and subsequent regeneration.

In a separate model of organ damage, we explored the recovery of the intestinal epithelium to irradiation. Canonical intestinal stem cells, marked by Lgr5 expression, reside at the base of intestinal crypts and renew the epithelium during normal homeostasis ${ }^{51}$. However, recent work has demonstrated that a rare, separate type of cell, named revival stem cells and marked by Clu expression, are responsible for regeneration in response to irradiation ${ }^{52}$. Consistent with our analysis in the Tabula Muris (see Fig 2), Lgr5+ intestinal stem cells display the highest transcript content amongst all cell types in the crypt compartment (Fig 5F-H). Interestingly, upon irradiation-induced loss of Lgr5+ intestinal stem cells, Clu+ revival stem cells assume comparably high levels of transcript content and cell cycling (Fig $\mathbf{5 H})$. These findings suggest that, as Clu+ revival stem cells exit quiescence to replenish the crypt niche, they enter hypertranscription to support the biosynthetic requirements of regenerative proliferation and differentiation. Thus, our results indicate that hypertranscription is redeployed in adult stem/progenitor cells both during physiological organ renewal as well as in different contexts of regeneration upon injury. 


\section{DISCUSSION}

We report here the development and validation of absolute-scaling approaches to estimate transcript abundance in scRNA-seq data. When applied to a variety of scRNAseq datasets, absolute scaling accurately captures known cases of hypertranscription in embryonic cells and uncovers a previously unappreciated rich level of global transcriptional dynamics in adult cells. Our analyses indicate that hypertranscription is pervasively redeployed by stem/progenitor cells across adult organ systems under both

502

503 homeostatic and regenerative conditions. Thus, rather than being largely ignored as it is by standard scRNA-seq analyses methods, we propose that hypertranscription is central to the biology of adult organs and needs to be considered in order to understand the mechanisms that underlie their maintenance and regeneration.

Our results document that absolute scaling is capable of capturing large-scale changes in transcriptional output in scRNA-seq data, as long as ERCC exogenous spike-ins or UMI barcodes are used. While both methods remain useful, our data slightly favor the use of ERCCs over UMls in this context, likely because they are introduced further upstream in sample processing. Nevertheless, several potential sources of technical variation remain. ERCC-based analysis is dependant on proper experimental aliquoting, and whether various technical biases equally affect endogenous and extrinsic sequences remains debated ${ }^{14}$. The accuracy of UMIs, in addition to aforementioned issues, can also be affected by fluctuations in sequencing depth ${ }^{14}$. As with all scRNAseq studies, the use of higher cell numbers and greater read depths per cell attenuate sources of potential artifactual noise. Nonetheless, our ground truth tests and reproduction of embryonic hypertranscription results strongly validate the ability of absolute scaling to detect hypertranscription and its associated features in scRNA-seq data. Our results underscore the notion that global variations in total cellular mRNA content represent an important but critically underappreciated dimension in many biological contexts. The capacity to explore hypertranscription in single-cell data will enrich our understanding of many biological questions, notably in adult organ maintenance, regeneration, pathology and malignancy. We anticipate that this will also be aided by the development of improved protocols that more quantitatively capture the composition of total cellular RNA, including ribosomal and non-coding RNA species.

528 Importantly, global scaling and absolute scaling are not mutually exclusive and can be applied to the same dataset, maximizing the information that is extracted from each experiment. Moreover, current methods for inference of cell trajectories in scRNA-seq data like pseudotime align the cells by transcriptome similarity but do not systematically annotate the start/end points of the trajectories, instead usually relying on manual

533 annotation ${ }^{53}$. We suggest that hypertranscription may be used to anchor the start of such trajectories, particularly in contexts of cell differentiation. In addition, hypertranscription provides a new dimension with which to refine heterogeneous cell populations that may express similar markers. This may be particularly useful to distinguish quiescent vs activated stem cells, as we observed for satellite cells in the muscle regeneration paradigm above (Fig. 5A-E). 
538 The existence of high transcript-content cells occupying adult stem cell and progenitor 539 niches expands the role of hypertranscription beyond development and tumorigenesis. Our findings are supported by early studies dating back to the 1930's, reporting evidence of elevated total RNA content within progenitor cells of the hematopoietic and intestinal systems ${ }^{54-56}$. In parallel, our findings of hypertranscription in the context of regeneration are closely aligned with classical studies showing increased RNA content in renewing cells of planarians and salamanders ${ }^{57,58}$. Our work builds upon these studies by comprehensively exploring transcriptional heterogeneity and dynamics at the single-cell and transcriptome-wide levels. In the process, our analyses uncover putative molecular drivers of hypertranscription in diverse contexts of adult organ homeostasis that warrant further studies.

We identify hypertranscription as a feature of active stem/progenitor cells that decays along lineage commitment. We note that the transcriptome is not linearly amplified or repressed, but follows patterns such as the primacy of modulation of regulators of ribogenesis and translation that are not fully understood and deserve further investigation $^{9,10}$. Moreover, transcript content does not decline strictly linearly down differentiation pathways but rather includes transient fluctuations, possibly due to high synthesis of differentiation effector proteins or expansion of transit amplifying-like cells (see Supp Fig 16). Further dissection of these transcriptional dynamics is likely to reveal new aspects of the biology of stem cell compartments. The existence of high transcript-content cells occupying adult stem cell and progenitor niches greatly expands the prevalence of hypertranscription beyond development and tumorigenesis ${ }^{2,13}$. Our initial analyses reveal many similarities between adult hypertranscription and its embryonic counterpart, but there are likely to be adult-specific and/or organ-specific aspects of the regulation of hypertranscription. It will be of interest to identify the primary drivers of the onset of and exit from hypertranscription in different organs, decipher the chromatin dynamics that underlie these changes, and dissect how they integrate with signaling from the stem/progenitor cell niche, both during physiological organ homeostasis and regeneration after injury. Our work lays a foundation for the investigation of hypertranscription in adult organs at the single cell level.

Early histochemical studies dating back to the 1950's reported evidence of elevated total RNA and protein content within renewing cells of planarians and salamanders, but

573 these findings have essentially been forgotten in the genomics era ${ }^{57,58}$. Our results put a new focus on hypertranscription and its molecular hallmarks that can now be applied to a plethora of regeneration paradigms being explored with scRNA-seq approaches, such as axolotl limb or mouse digit tip regeneration ${ }^{59-62}$. Of note, it has recently been shown

576

577

578 that during generation of iPS cells a combination of high proliferative capacity with hypertranscription identifies cells with the greatest probability to faithfully reprogram to pluripotency ${ }^{63}$. We anticipate that further exploration of hypertranscription in the context of regeneration and cellular reprogramming paradigms will provide fundamental new molecular insights that may have broad impact in regenerative medicine. 


\section{METHODS}

583 Datasets and software versions used for this study are outlined in Supp Fig 1C. Raw read data from published studies were downloaded from either ENA or SRA. These include ArrayExpress accession code E-MTAB-2600 25 and Gene Expression Omnibus accession codes GSE11876723, GSE9658321, GSE10831320, GSE13882650, GSE136441 ${ }^{26}$, GSE109774 ${ }^{31}$, and GSE123516 ${ }^{52}$. The 12K 1:1 HEK293T-NIH3T3 mixture dataset was obtained from 10X Genomics under the Chromium v2 Chemistry Demonstration.

590

\section{Pre-Processing of scRNA-seq Data}

593

594

595

Detailed parameters for analysis and figure scripts are provided at

https://github.com/yunkyokim/AbsoluteScaling. Published count matrices with collapsed UMls or mapped ERCC sequences were used where available. For CEL/Sort-seq data from Tian et al 2019, new count matrices were generated using the scPipe pipeline using genomic references containing ERCC sequence data ${ }^{64}$. Spliced/unspliced count matrices for the bone marrow, intestine, and skin FACS datasets were generated using the scVelo pipeline for analysis of nascent reads ${ }^{65}$.

601

Seurat objects were used for processing of all scRNA-seq data. Low-quality cells in

602

603

604

605

606

607

608

609

610

611 individual organ datasets were filtered by mitochondrial gene expression, ERCC fraction, and gene/transcript counts. To address the possibility that high-content cells were the result of libraries generated from doublets, we performed heterotypic doublet removal on Smartseq2 and 10X Genomics datasets using DoubletFinder ${ }^{32}$. The total doublet rate was estimated at $4 \%$, and the homotypic doublet rate was derived from Tabula Muris cell-type annotations. All doublet removal was performed on globallyscaled data with standard processing.

\section{Scaling and Dimensional Reduction}

Absolute scaling analysis of ERCC datasets was performed using the scran package ${ }^{66}$.

612 To ensure that transcript counts between organs were comparable, individual organ

613

614

615

616

617

618

619

620 count matrices were merged and per-cell size factors were generated from spike-in data using the computeSpikeFactors function. This calculation is derived from the sum of all spike-in reads and generates a set of size factors with a mean of unity. Single-cell libraries were then normalized to size factors and log2 scaled. For absolute scaling analysis of UMI datasets, raw UMI counts were used following log2 scaling. Global scaling was performed on ERCC and UMI datasets by first removing spike-in data (if present) and normalizing for library size using the relative counts methods in Seurat's NormalizeData function. Results were subsequently log2 scaled and used for analysis.

621

622

623

624

Standard dimensional reduction was performed using Seurat functions with detailed parameters available in code. Briefly, highly variable features were scaled and used for principal component analysis, with the number of components determined using

625 resampling tests ${ }^{67}$. Data was subsequently visualized using the Uniform Approximation and Projection Method (UMAP). 
627 Cell Cycle Scoring and Signature Analysis

628 To determine cell phase, we used the CellCycleScoring method from Seurat with

629 markers as previously published ${ }^{35}$. Briefly, individual cells were scored based on the

630 expression of either G2/M or S phase cell markers. Cells lacking expression of either

631 marker set were identified to be in a non-cycling G1 phase. For signature analysis, we

632 used the VISION tool to calculate signature scores using gene sets derived from the

633 Molecular Signatures Database ${ }^{68,69}$. Signatures were calculated using absolute-scaled

634 data without internal transformation within VISION. For the "Serum Hypertranscription"

635 signature, >2.5-fold differentially upregulated genes in serum mESCs (Kolodziejczyk et

636 al 2015) were used to generate the gene set.

637

638

\section{Quantile Polarization Scores and Pseudotemporal Ordering}

639 Quantile polarization scores were calculated with VECTOR using the vector.getValue

640 function. As previously described, we determined quantile polarization values on

641 absolute-scaled data using the top 150 principal components ${ }^{44}$. For pseudotemporal

642 analysis with Monocle 3, we converted pre-processed and absolute-scaled Seurat

643 objects into Monocle3 cds objects. Cells were clustered on previously generated UMAP

644 reductions using the Leiden algorithm. To construct single-cell trajectories,

645 hematopoietic stem cells expressing Cd34 and Kit were used as root cells. Principal

646 graphs were constructed and used for pseudotemporal ordering.

Quantification and Statistical Analysis

649

650

All statistical analysis was performed using functions implemented in R. Spearman correlation was used to identify signatures related to cellular transcript abundance and was calculated using cor. Transcriptome curves were drawn using geom smooth in ggplot2 using a "loess" model. Cumulative distributions plots for mESC transcriptomes were generated using ecdf. For differential gene expression in scRNA-seq data, we

654 used the FindMarkers functions with Seurat and performed gene ontology and ChEA 655 enrichment analysis in Enrichr ${ }^{70}$. 


\section{ACKNOWLEDGMENTS}

657 We thank Gary Bader, Aydan Bulut-Karslioglu, Kieran Campbell, Joshua Currie,

658 Michelle Percharde, and members of the Santos Lab for input and critical reading of the

659 manuscript. This work is supported by a Whiteside Scholarship (University of Toronto)

660 and a McLaughlin Award (University of Toronto) to Y.K., CIHR Project Grant 420231,

661 and a Canada 150 Research Chair in Developmental Epigenetics to M.R.-S.

662 
bioRxiv preprint doi: https://doi.org/10.1101/2021.12.13.472426; this version posted December 13, 2021. The copyright holder for this preprint (which was not certified by peer review) is the author/funder. All rights reserved. No reuse allowed without permission.

\section{AUTHOR CONTRIBUTIONS}

664 Y.K. and M.R-S. conceived of the project. Y.K. performed all analyses. M.R-S.

665 supervised the project. Y.K. and M.R.-S. wrote the manuscript.

666 
bioRxiv preprint doi: https://doi.org/10.1101/2021.12.13.472426; this version posted December 13, 2021. The copyright holder for this preprint (which was not certified by peer review) is the author/funder. All rights reserved. No reuse allowed without permission.

\section{COMPETING INTERESTS}

668 The authors declare no competing interests.

669 
671 Figure 1: Detection of Embryonic Hypertranscription by Absolute Scaling

672 (A-B) UMAP visualization of serum/2i mESCs under global/absolute scaling. Plots

673 depict dimensionality reduction performed under the indicated scaling type.

674

675 (C-F) Total transcript counts in serum/2i mESCs under global/absolute scaling. Counts

676 in (C), (E) represent sums of globally scaled transcripts following log2 scaling, while counts in (D), (F) represent total transcripts following ERCC normalization. Lines correspond to median values.

678 (G-H) Cumulative distribution plots depicting gene expression across top 25,000 genes in serum/2i mESCs. Distributions were computed using log2 expression in both conditions.

682 (I-J) Enriched GO terms from $\geq 2$-fold differentially upregulated genes in serum mESCs

683 under global or absolute scaling conditions.

684 (K-L) UMAP visualization of E12.5 gonad scRNA-seq data under global/absolute scaling containing Oct4+ PGCs and Oct4- soma. Plots depict dimensionality reduction performed under the indicated scaling type.

686

687

688 (M-P) Transcript abundance in gonad scRNA-seq data under global/absolute scaling. Counts in (M), (O) represent sums of globally scaled UMls following log2 scaling, while counts in $(\mathbf{N}),(\mathbf{P})$ represent raw UMls. Lines correspond to median values and are coloured according to cell type.

691 (Q) Cell cycle scoring of gonad cells. Phase scores were calculated using cell cycle

692

693

694

695 markers from globally-scaled gene expression data. Lines correspond to median values and are coloured according to cell type.

696

697

698

Figure 2: Absolute Scaling Reveals Transcriptional Content Heterogeneity in the Tabula Muris

(A-B) Distribution of cellular transcript content between FACS organ datasets under absolute scaling, ranked by median total transcripts. Transcripts represent ERCCnormalized reads, lines correspond to median values.

699

700 (C-E) Single-cell correlation of transcription signatures with log2 transcript abundance.

701

702

703

704

705

706

707

708

709

710

711 Signature scores were determined using VISION with absolute-scaled FACS data across the entire atlas. Each point represents a single cell, with color scale depicting plotting density.

(F) Ranking of genes by Spearman coefficient to log2 transcript abundance across the entire FACS atlas. Highlighted genes represent all expressed Rpl and Rps genes.

(G) Correlation between Chd1 expression with log2 transcript abundance. Each point is representative of a single averaged cell type, with colors matching organs in (A). Correlation value represents Spearman coefficient.

(H) Enrichment analysis for GO biological process terms using top 3000 highly correlated genes (to log2 transcript abundance) under absolute scaling.

712

713

714

715

Figure 3: Hematopoietic Progenitors Display Hallmarks of Hypertranscription (A) UMAP visualization of cell types within the bone marrow dataset using dimensionality reduction performed under absolute scaling.

(B) Distribution of cellular transcript content between cell types of the bone marrow FACS dataset. Transcript counts represent log2 ERCC-normalized reads. 
(C) Transcriptome curves depicting gene expression across top 20,000 genes in representative cell types of the bone marrow. Individual genes are ranked using combined log2 expression between all cell types. Indicated cell types correspond to the highlighted curve.

(D) Expression of select genes relevant to hallmarks of hypertranscription, including (1) chromatin remodelers, (2) DNA repair factors, (3) ribosomal genes, (4) housekeeping genes, and (5) hematopoietic markers.

(E) UMAP visualization of signature scores generated by VISION using absolute-scaled expression data.

(F) UMAP visualization of total spliced/unspliced transcripts and Chd1 expression under absolute scaling. Unspliced or nascent transcripts were assigned by the detection of intronic sequences.

Figure 4: Hypertranscription Marks Developmental Progress in Hematopoiesis under absolute scaling. Cd34+/cKit+ hematopoietic stem cells were defined as root cells for trajectory inference analysis.

733

734 (C) Heatmap depicting transcriptomic expression changes across differentiation pseudotime from hematopoietic stem cell to granulocyte. Rows depict individual genes and columns depict single-cell transcriptomes ordered by pseudotime values. Class annotations represent k-means clusters with expression presented as z-scores. (D-I) Transcriptome curves representing expression of clustered genes across progression of pseudotime. Dotted line represents average expression of all genes (as depicted in (C)). Right panels of each column show enriched GO terms derived from genes within each class. Expression in (C-I) represents ranged log2 expression of ERCC-normalized reads. annotated satellite cells across all time points. Plot depicts dimensionality reduction performed under absolute scaling.

(B) UMAP visualization of muscle satellite cells. Plot depicts dimensionality reduction performed under absolute scaling.

(C) Distribution of transcript content between cell types across all time points. Lines correspond to median values.

(D) Progression of satellite cell transcript content and cell number through injury and regeneration. Transcript counts represent raw UMls, error bars represent standard deviation.

(E) GO terms enriched in >1.5-fold differentially upregulated genes in 0.5 DPI vs noninjured satellite cells.

(F-G) UMAP visualization of transcript abundance, stem cell marker Lgr5, and revival stem cell marker Clu across all cells in normal/irradiated crypts. Plots depict

759 dimensionality reduction performed under absolute scaling. 
bioRxiv preprint doi: https://doi.org/10.1101/2021.12.13.472426; this version posted December 13, 2021. The copyright holder for this preprint (which was not certified by peer review) is the author/funder. All rights reserved. No reuse allowed without permission.

760 (H) Distribution of transcript abundance and cell-phase scores between cell types in 761 normal and irradiated crypts. Cell types are ranked by median log2 UMls with lines 762 corresponding to median values.

763 
(A) Schematic of ERCC and UMI tools within scRNA-seq protocols. Tissue samples are disassociated into single cells and lysed to capture mRNA transcripts. ERCC spike-in sequences are added to lysed contents and captured alongside endogenous mRNA species. UMls are added during the capture and RT steps to uniquely label transcripts. (B) Model of hypertranscription in scRNA-seq datasets. Absolute scaling preserves large-scale transcriptomic shifts that are typically masked in global scaling approaches due to assumptions of mRNA content parity between cells of a dataset.

(C) Summary of bioinformatic tools and datasets used for absolute scaling in this study.

776

\section{Supplementary Figure 2: Absolute Scaling Reproduces Transcript Content Differences Between Singlets and Doublets}
(A) Transcript abundances in singlets and doublets within ground truth datasets. Transcripts represent raw UMIs, lines correspond to median values.

(B) Dot plots depicting expression of ribosomal/housekeeping genes under absolute scaling in PBMC ground truth datasets. Expression represents z-scores of log2-scaled raw UMls.

(C) Average expression of RPL and RPS gene expression in ground truth PBMC datasets. Lines correspond to median values.

790

791

792

793

794

795

796

797

\section{Supplementary Figure 3: Absolute Scaling Reproduces Pseudocell Transcript Content in Benchmarking Mixtures}

(A, C) Transcript abundances per pseudocell vs ground truth mRNA content under different normalization methods. Each point represents a single artificially-generated pseudo-cell transcriptome using defined amounts of cell-line mRNA.

\section{(B, D) Transcriptome curves depicting gene expression across top 25,000 genes in} pseudocells. Individual genes are ranked using combined log2 expression between all pseudocell ground truth conditions.

(E, F) Comparison of UMI vs ERCC absolute scaling. Ranged expression represents log2 raw UMIs or ERCC-normalized reads.

\section{Supplementary Figure 4: Absolute Scaling Captures Hypertranscription Hallmarks in Embryonic Datasets}

798 (A-B) Expression of genes relevant to hypertranscription hallmarks in serum/2i mESCs 799 under global vs absolute scaling.

800 (C-D) Expression of genes relevant to hypertranscription hallmarks in Oct4+

801 PGCs/Oct4- soma under global vs absolute scaling. Expression represents z-scores of 802 log2-scaled transcripts.

803

804

805

806

807

Supplementary Figure 5: Transcript Content Heterogeneity in Tabula Muris 10X Datasets

808

(A-B) Distribution of cellular transcript content between Droplet organ datasets under absolute scaling, ranked by median total transcripts. Transcripts represent raw UMIs, lines correspond to median values. 
(C-D) UMAP representations of FACS/Droplet Tabula Muris Atlas generated under globally-scaled gene expression. Transcript abundance represents log2 scaled ERCCnormalized reads (C) or log2 absolute UMIs (D). Plots depict dimensionality reduction performed under absolute scaling. (A-F) Transcript and feature counts of Tabula Muris FACS or Droplet atlases. (G) Linear correlations of ERCC spike-in expression between FACS organ datasets. Values represent Pearson coefficients.

820

(H) Representation of filtering in FACS datasets for transcript counts and ERCC percentage.

821

(I) Comparison of actual and expected ERCC species expression based on spike-in mix composition.

822 (J) Comparison of individual gene expression between FACS and Droplet datasets. Each point represents a single shared gene between FACS and Droplet dataset. (K) Comparison of average transcript abundance in organ datasets between FACS and Droplet atlases. For (J-K), transcripts in FACS represents log2 ERCC-normalized expression, while values in Droplet represent log2 absolute UMls. Correlation values represent Pearson coefficients.

\section{Supplementary Figure 7: Absolute Scaling Reveals Clustering of High-Transcript Content Cells}

(A-B) UMAP representations of FACS/Droplet organ datasets using dimensionality reduction under globally-scaled gene expression. Transcript abundance represents log2 scaled ERCC-normalized reads (A) or log2 absolute UMls (B).

840

841

842

843

844

\section{Supplementary Figure 8: Shared Cell Types Between Organ Datasets Display} Similar Transcript Abundances

(A-B) Boxplots of transcript content and UMAP visualizations of shared cell types in FACS (A) or Droplet (B) datasets. Cell types ranked by median transcript content. Values in (A) represent log2 scaled ERCC-normalized reads, while values in (B) represent log2 absolute UMls.

\section{Supplementary Figure 9: Cell Cycle Phase Distribution in Across FACS Atlas}

845

846

847

(A) Cell cycle phase proportions across all cell types, sorted by percentage of G2/M and $S$ cells. Phase scores were calculated using globally-scaled expression data.

848

849

(B) Transcript abundance across all cell types, sorted by percentage of G2M and S cells. Transcripts represent log2 scaled ERCC-normalized reads. Line represents moving average across 7 cell types.

850

(C) Comparison between aggregate $\mathrm{G} 2 \mathrm{M} / \mathrm{S}$ score and transcript abundance across all cells.

852

Supplementary Figure 10: Cell Cycle Phase Distribution in Across Droplet Atlas

853

(A) Cell cycle phase proportions across all cell types, sorted by percentage of G2/M and $\mathrm{S}$ cells. Phase scores were calculated using globally-scaled expression data. 
854 (B) Transcript abundance across all cell types, sorted by percentage of G2M and S

855 cells. Transcripts represent log2 absolute UMls. Line represents moving average across

8567 cell types.

857 (C) Comparison between aggregate G2M/S score and transcript abundance across all

858 cells.

\section{Supplementary Figure 11: High-Content Cells of the Liver and Bladder Display} liver polyploid cell markers. Plots depict dimensionality reduction performed under

864 absolute scaling. (E-O) ERCC-normalized transcript abundance and log2 expression of polyploid cell markers in bladder cells. Plots depict dimensionality reduction performed under the indicated scaling type.

\section{Supplementary Figure 12: Hypertranscription Hallmarks are Correlated with} Transcript Abundance

(A-C) Single-cell correlation of transcription signatures with log2 transcript abundance. Signature scores were determined using VISION with absolute-scaled FACS data. Each point represents a single cell, with color scale depicting plotting density. (D-I) Positive and negative correlation of select gene expression with log2 transcript abundance. Cells are displayed as averages within cell types, with colors matching organs in (Fig 2). Correlation values represent Spearman coefficients. transcript abundance).

\section{Supplementary Figure 13: Transcript Content is a Correlated to Markers of} Developmental Progression

(A) Comparison of log2 absolute-scaled transcript content with feature counts (number of expressed genes).

(B) Comparison of log2 absolute-scaled transcript content with quantile progression scores, calculated using globally-scaled FACS data. Correlation values in (A-B) represent Spearman coefficients.

890

891

892

\section{Supplementary Figure 14: Epidermal Progenitors Display Hallmarks of} Hypertranscription

893

(A) UMAP visualization of cell types within the skin FACS dataset.

(B) Visualization of cycling Top2a+ epidermal stem cells within the dataset. Plots in (AB) depict dimensionality reduction performed under absolute scaling.

(C) Transcript content differences between Top2a+ and Top2a- cells

(D) Distribution of cellular transcript content between cell types of the epidermis, ranked by median content. Transcript counts represent log2 ERCC-normalized reads. representative cell types of the epidermis. Individual genes are ranked using combined 
(F) Expression of select genes relevant to hallmarks of hypertranscription, including (1) chromatin remodelers, (2) DNA repair factors, (3) ribosomal genes, (4) housekeeping

903 genes, and (5) epidermal compartment markers.

904 (G) UMAP visualization of total spliced/unspliced transcripts, Chd1 and Myc expression under absolute scaling.

906

907

(H) UMAP visualization of signature scores generated by VISION using absolute

908

909

910 scaling.

911

\section{Supplementary Figure 15: Colonic Epithelium Progenitors Display Hallmarks of} Hypertranscription

912

913

(A) UMAP visualization of cell types within the large intestine FACS dataset.

(B) Visualization of cycling Lgr5+ intestinal stem cells within the dataset. Plots in (A-B)

914

915

916 depict dimensionality reduction performed under absolute scaling.

(C) Transcript content between Lgr5+ and Lgr5- cells.

(D) Distribution of cellular transcript content between cell types of the colonic epithelium.

917

918

919

920 Transcript counts represent log2 ERCC-normalized reads.

(E) Transcriptome curves depicting gene expression across top 20,000 genes in representative cell types of the colonic epithelium. Individual genes are ranked using combined log2 expression between all cell types. Indicated cell types correspond to the highlighted curve.

921

922

923

(F) Expression of select genes relevant to hallmarks of hypertranscription, including (1) chromatin remodelers, (2) DNA repair factors, (3) ribosomal genes, (4) housekeeping genes, and (5) crypt cell markers.

924

925

(G) UMAP visualization of total spliced/unspliced transcripts, Chd1 and Myc expression under absolute scaling.

926

927

928

929

(H) UMAP visualization of signature scores generated by VISION using absolute scaling.

930

\section{Supplementary Figure 16: Hypertranscription Marks Developmental Progress}

931

932 Through Erythroblast and Monocyte Differentiation

933

(A) UMAP representation of cell types within Tabula Muris 10X bone marrow dataset.

(B) Transcript abundance (raw UMls) across all cells of the bone marrow.

934

(C-D, I-J) UMAP visualization of Monocle pseudotime scores and total transcript

935 abundance under absolute scaling. Hematopoietic stem cells were defined as root cells for analysis. Plots in (A-B), (C-D), and (I-J) depict dimensionality reduction performed

\section{(E-H, K-N) Gene curves}

937 (E-H, K-N) Gene curves representing absolute-scaled expression of indicated genes

938 through progression of pseudotime. Each point represents single cells ordered by

939 pseudotime values.

940 (O) Gene curves representing globally-scaled expression of indicated genes through

941 progression of pseudotime. Each point represents single cells ordered by pseudotime

942 values. Expression values in $(\mathbf{E}, \mathbf{K})$ represent raw UMls, values in $(\mathbf{F}-\mathbf{H}, \mathbf{L}-\mathbf{N})$ represent

$943 \log 2$ raw UMIs.

944 


\section{SUPPLEMENTARY TABLES}

946 Table S1: Absolute Scaling Statistics for Tabula Muris Atlas

947

948 Table S2: Gene-Transcript Content Correlations

949

950 Table S3: GO BP and ChEA Terms Enriched in Highly Correlated Genes 


\section{REFERENCES}

952

1. Lane, A. N. \& Fan, T. W. M. Regulation of mammalian nucleotide metabolism and

953

954 biosynthesis. Nucleic Acids Res. 43, 2466-2485 (2015).

955

956

957

958

959

960

961

962

963

964

965

966

967

968

969

970

971

972

973

974

975

976

977

978

979

980

981

982

983

984

985

986

987

988

989

990

991

992

993

2. Percharde, M., Bulut-Karslioglu, A. \& Ramalho-Santos, M. Hypertranscription in Development, Stem Cells, and Regeneration. Dev. Cell 40, 9-21 (2017).

3. Percharde, M., Wong, P. \& Ramalho-Santos, M. Global Hypertranscription in the Mouse Embryonic Germline. Cell Rep. 19, 1987-1996 (2017).

4. Guzman-Ayala, M. et al. Chd1 is essential for the high transcriptional output and rapid growth of the mouse epiblast. Dev. 142, 118-127 (2015).

5. Koh, F. M. et al. Emergence of hematopoietic stem and progenitor cells involves a Chd1-dependent increase in total nascent transcription. Proc. Natl. Acad. Sci. U. S. A. 112, E1734-E1743 (2015).

6. Lin, C. Y. et al. Transcriptional amplification in tumor cells with elevated c-Myc. Cell 151, 56-67 (2012).

7. Nie, Z. et al. c-Myc is a universal amplifier of expressed genes in lymphocytes and embryonic stem cells. Cell 151, 68-79 (2012).

8. Lavado, A. et al. The Hippo Pathway Prevents YAP/TAZ-Driven Hypertranscription and Controls Neural Progenitor Number. Dev. Cell 47, 576591.e8 (2018).

9. Bulut-Karslioglu, A. et al. Inhibition of $\mathrm{mTOR}$ induces a paused pluripotent state. Nature 540, 119-123 (2016).

10. Bulut-Karslioglu, A. et al. The Transcriptionally Permissive Chromatin State of Embryonic Stem Cells Is Acutely Tuned to Translational Output. Cell Stem Cell 22, 369-383.e8 (2018).

11. Gaspar-Maia, A. et al. Chd1 regulates open chromatin and pluripotency of embryonic stem cells. Nature 460, 863-868 (2009).

12. Bulut-Karslioglu, A. et al. Chd1 protects genome integrity at promoters to sustain hypertranscription in embryonic stem cells. Nat. Commun. 12, 1-11 (2021).

13. Wolf, E., Lin, C. Y., Eilers, M. \& Levens, D. L. Taming of the beast: Shaping Mycdependent amplification. Trends Cell Biol. 25, 241-248 (2015).

14. Vallejos, C. A., Risso, D., Scialdone, A., Dudoit, S. \& Marioni, J. C. Normalizing single-cell RNA sequencing data: Challenges and opportunities. Nat. Methods 14, 565-571 (2017).

15. Bacher, R. \& Kendziorski, C. Design and computational analysis of single-cell RNA-sequencing experiments. Genome Biol. 17, 1-14 (2016).

16. Trapnell, C. \& Liu, S. Single-cell transcriptome sequencing: Recent advances and remaining challenges. F1000Research 5, (2016).

17. Klein, A. M. et al. Droplet barcoding for single-cell transcriptomics applied to embryonic stem cells. Cell 161, 1187-1201 (2015).

18. Islam, S. et al. Quantitative single-cell RNA-seq with unique molecular identifiers. Nat. Methods 11, 163-166 (2014).

19. Ziegenhain, C., Vieth, B., Parekh, S., Hellmann, I. \& Enard, W. Quantitative single-cell transcriptomics. Brief. Funct. Genomics 17, 220-232 (2018).

994

20. Stoeckius, M. et al. Cell Hashing with barcoded antibodies enables multiplexing and doublet detection for single cell genomics. Genome Biol. 19, 1-12 (2018).

996

21. Kang, H. M. et al. Multiplexed droplet single-cell RNA-sequencing using natural 
997

998

999

1000

1001

1002

1003

1004

1005

1006

1007

1008

1009

1010

1011

1012

1013

1014

1015

1016

1017

1018

1019

1020

1021

1022

1023

1024

1025

1026

1027

1028

1029

1030

1031

1032

1033

1034

1035

1036

1037

1038

1039

1040

1041

1042

genetic variation. Nat. Biotechnol. 36, 89-94 (2018).

22. Chen, Y. et al. Dynamic interactions and intracellular fate of label-free, thin graphene oxide sheets within mammalian cells: role of lateral sheet size.

Nanoscale Adv. 3, 4166-4185 (2021).

23. Tian, L. et al. Benchmarking single cell RNA-sequencing analysis pipelines using mixture control experiments. Nat. Methods 16, 479-487 (2019).

24. Lim, P. S. L. \& Meshorer, E. Organization of the pluripotent genome. Cold Spring Harb. Perspect. Biol. 13, 1-23 (2021).

25. Kolodziejczyk, A. A. et al. Single Cell RNA-Sequencing of Pluripotent States Unlocks Modular Transcriptional Variation. Cell Stem Cell 17, 471-485 (2015).

26. Niu, W. \& Spradling, A. C. Two distinct pathways of pregranulosa cell differentiation support follicle formation in the mouse ovary. Proc. Natl. Acad. Sci. U. S. A. 117, 20015-20026 (2020).

27. Tam, P. P. \& Snow, M. H. Proliferation and migration of primordial germ cells during compensatory growth in mouse embryos. J. Embryol. Exp. Morphol. 64, 133-147 (1981).

28. Han, X. et al. Construction of a human cell landscape at single-cell level. Nature 581, 303-309 (2020).

29. Han, X. et al. Mapping the Mouse Cell Atlas by Microwell-Seq. Cell 172, 10911107.e17 (2018).

30. Cao, J. et al. A human cell atlas of fetal gene expression. Science (80-. ). 370, (2020).

31. Schaum, N. et al. Single-cell transcriptomics of 20 mouse organs creates a Tabula Muris. Nature 562, 367-372 (2018).

32. McGinnis, C. S., Murrow, L. M. \& Gartner, Z. J. DoubletFinder: Doublet Detection in Single-Cell RNA Sequencing Data Using Artificial Nearest Neighbors. Cell Syst. 8, 329-337.e4 (2019).

33. Sender, R. \& Milo, R. The distribution of cellular turnover in the human body. Nat. Med. 27, 45-48 (2021).

34. Jao, C. Y. \& Salic, A. Exploring RNA transcription and turnover in vivo by using click chemistry. Proc. Natl. Acad. Sci. U. S. A. 105, 15779-15784 (2008).

35. Kowalczyk, M. S. et al. Single-cell RNA-seq reveals changes in cell cycle and differentiation programs upon aging of hematopoietic stem cells. Genome Res. 25, 1860-1872 (2015).

36. Wang, J. et al. Polyploid Superficial Cells that Maintain the Urothelial Barrier Are Produced via Incomplete Cytokinesis and Endoreplication. Cell Rep. 25, 464477.e4 (2018).

37. Celton-Morizur, S. \& Desdouets, C. Polyploidization of liver cells. Adv. Exp. Med. Biol. 676, 123-135 (2010).

38. Martin, N. C. et al. Functional analysis of mouse hepatocytes differing in DNA content: Volume, receptor expression, and effect of IFNy. J. Cell. Physiol. 191, 138-144 (2002).

39. Donne, R., Saroul-Aïnama, M., Cordier, P., Celton-Morizur, S. \& Desdouets, C. Polyploidy in liver development, homeostasis and disease. Nat. Rev. Gastroenterol. Hepatol. 17, 391-405 (2020).

40. Wang, M. J., Chen, F., Lau, J. T. Y. \& Hu, Y. P. Hepatocyte polyploidization and 
1043

1044

1045

1046

1047

1048

1049

1050

1051

1052

1053

1054

1055

1056

1057

1058

1059

1060

1061

1062

1063

1064

1065

1066

1067

1068

1069

1070

1071

1072

1073

1074

1075

1076

1077

1078

1079

1080

1081

1082

1083

1084

1085

1086

1087

1088 its association with pathophysiological processes. Cell Death Dis. 8, e2805 (2017).

41. Ni, V. V, Shteĭn, G. I., Mă̌tesian, E. S. \& Kudriavtsev, B. N. [The RNA content of hepatocytes of different ploidies]. Tsitologiia 30, 354-358 (1988).

42. Richter, M. L. et al. Single-nucleus RNA-seq2 reveals functional crosstalk between liver zonation and ploidy. Nat. Commun. 12, 1-16 (2021).

43. Keenan, A. B. et al. ChEA3: transcription factor enrichment analysis by orthogonal omics integration. Nucleic Acids Res. 47, W212-W224 (2019).

44. Zhang, F., Li, X. \& Tian, W. Unsupervised Inference of Developmental Directions for Single Cells Using VECTOR. Cell Rep. 32, 108069 (2020).

45. Gulati, G. S. et al. Single-cell transcriptional diversity is a hallmark of developmental potential. Science 367, 405-411 (2020).

46. Jagannathan-Bogdan, M. \& Zon, L. I. Hematopoiesis. Development 140, 24632467 (2013).

47. Barker, N. et al. Identification of stem cells in small intestine and colon by marker gene Lgr5. Nature 449, 1003-1007 (2007).

48. Cao, J. et al. The single-cell transcriptional landscape of mammalian organogenesis. Nature 566, 496-502 (2019).

49. Yin, H., Price, F. \& Rudnicki, M. A. Satellite cells and the muscle stem cell niche. Physiol. Rev. 93, 23-67 (2013).

50. Oprescu, S. N., Yue, F., Quu, J., Brito, L. F. \& Kuang, S. Temporal Dynamics and Heterogeneity of Cell Populations during Skeletal Muscle Regeneration. iScience 23, 100993 (2020).

51. Zhu, G., Hu, J. \& Xi, R. The cellular niche for intestinal stem cells: a team effort. Cell Regen. 10, 1 (2021).

52. Ayyaz, A. et al. Single-cell transcriptomes of the regenerating intestine reveal a revival stem cell. Nature 569, 121-125 (2019).

53. Saelens, W., Cannoodt, R., Todorov, H. \& Saeys, Y. A comparison of single-cell trajectory inference methods. Nat. Biotechnol. 37, 547-554 (2019).

54. Thornell, B. Studies on the Formation of Cellular Substances During Blood Cell Production. J. Am. Med. Assoc. 137, 1265 (1948).

55. Caspersson, T. \& Schultz, J. Pentose Nucleotides in the Cytoplasm of Growing Tissues. Nature 143, 602-603 (1939).

56. Jao, C. Y. \& Salic, A. Exploring RNA transcription and turnover in vivo by using click chemistry. Proc. Natl. Acad. Sci. U. S. A. 105, 15779-15784 (2008).

57. Bodemer, C. W. Distribution of ribonucleic acid in the regenerating urodele limb as determined by autoradiographic localization of uridine-H3. Anat. Rec. 142, 457-467 (1962).

58. Bodemer, C. W. \& Everett, N. B. Localization of newly synthesized proteins in regenerating newt limbs as determined by radioautographic localization of injected methionine-S35. Dev. Biol. 1, 327-342 (1959).

59. Leigh, N. D. et al. Transcriptomic landscape of the blastema niche in regenerating adult axolotl limbs at single-cell resolution. Nat. Commun. 9, 5153 (2018).

60. $\mathrm{Li}, \mathrm{H}$. et al. Dynamic cell transition and immune response landscapes of axolotl limb regeneration revealed by single-cell analysis. Protein \& cell vol. 12 57-66 (2021). 
1089

1090

1091

1092

1093

1094

1095

1096

1097

1098

1099

1100

1101

1102

1103

1104

1105

1106

1107

1108

1109

1110

1111

1112

1113

1114

1115

1116

61. Gerber, T. et al. Single-cell analysis uncovers convergence of cell identities during axolotl limb regeneration. Science 362, (2018).

62. Storer, M. A. et al. Acquisition of a Unique Mesenchymal Precursor-like Blastema State Underlies Successful Adult Mammalian Digit Tip Regeneration. Dev. Cell 52, 509-524.e9 (2020).

63. Babos, K. N. et al. Mitigating Antagonism between Transcription and Proliferation Allows Near-Deterministic Cellular Reprogramming. Cell Stem Cell 25, 486500.e9 (2019).

64. Tian, L. et al. scPipe: A flexible R/Bioconductor preprocessing pipeline for singlecell RNA-sequencing data. PLoS Comput. Biol. 14, 1-15 (2018).

65. Bergen, V., Lange, M., Peidli, S., Wolf, F. A. \& Theis, F. J. Generalizing RNA velocity to transient cell states through dynamical modeling. Nat. Biotechnol. 38, 1408-1414 (2020).

66. Lun, A. T. L., McCarthy, D. J. \& Marioni, J. C. A step-by-step workflow for lowlevel analysis of single-cell RNA-seq data with Bioconductor [version 2; peer review: 3 approved, 2 approved with reservations]. F1000Research 5, (2016).

67. Macosko, E. Z. et al. Highly Parallel Genome-wide Expression Profiling of Individual Cells Using Nanoliter Droplets Resource Highly Parallel Genome-wide Expression Profiling of Individual Cells Using Nanoliter Droplets. Cell 161, 12021214 (2015).

68. DeTomaso, D. et al. Functional interpretation of single cell similarity maps. Nat. Commun. 10, (2019).

69. Subramanian, A. et al. Gene set enrichment analysis: A knowledge-based approach for interpreting genome-wide expression profiles. Proc. Natl. Acad. Sci. U. S. A. 102, 15545-15550 (2005).

70. Chen, E. Y. et al. Enrichr: interactive and collaborative HTML5 gene list enrichment analysis tool. BMC Bioinformatics 14, 128 (2013). 
bioRxiv preprint doi: https://doi.org/10.1101/2021.12.13.472426; this version posted December 13, 2021. The copyright holder for this preprint (which was not certified by peer review) is the author/funder. All rights reserved. No reuse allowed without permission.
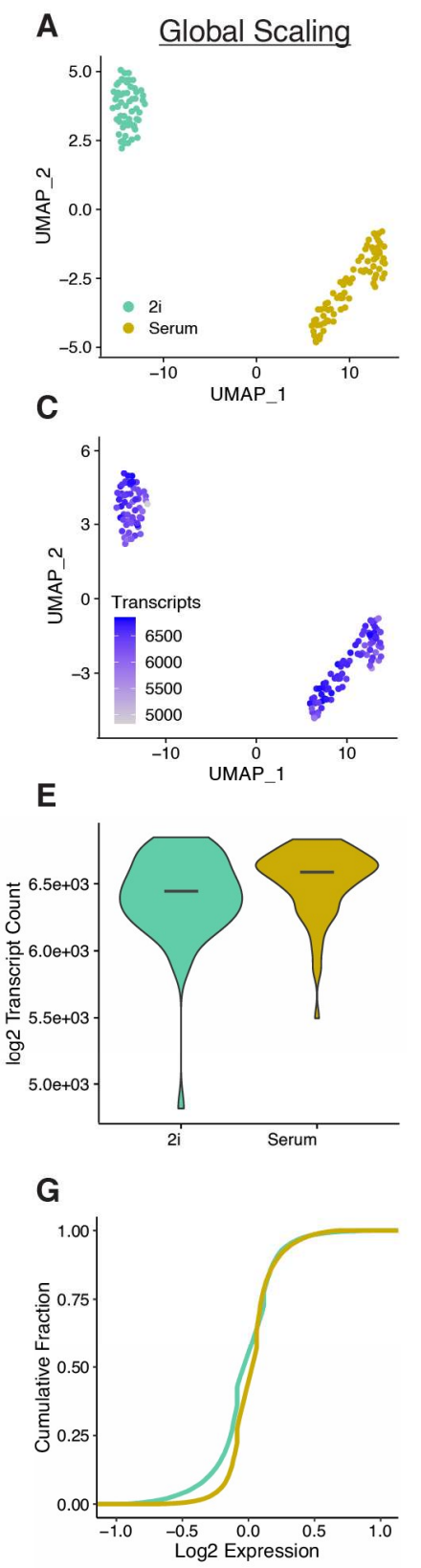

I
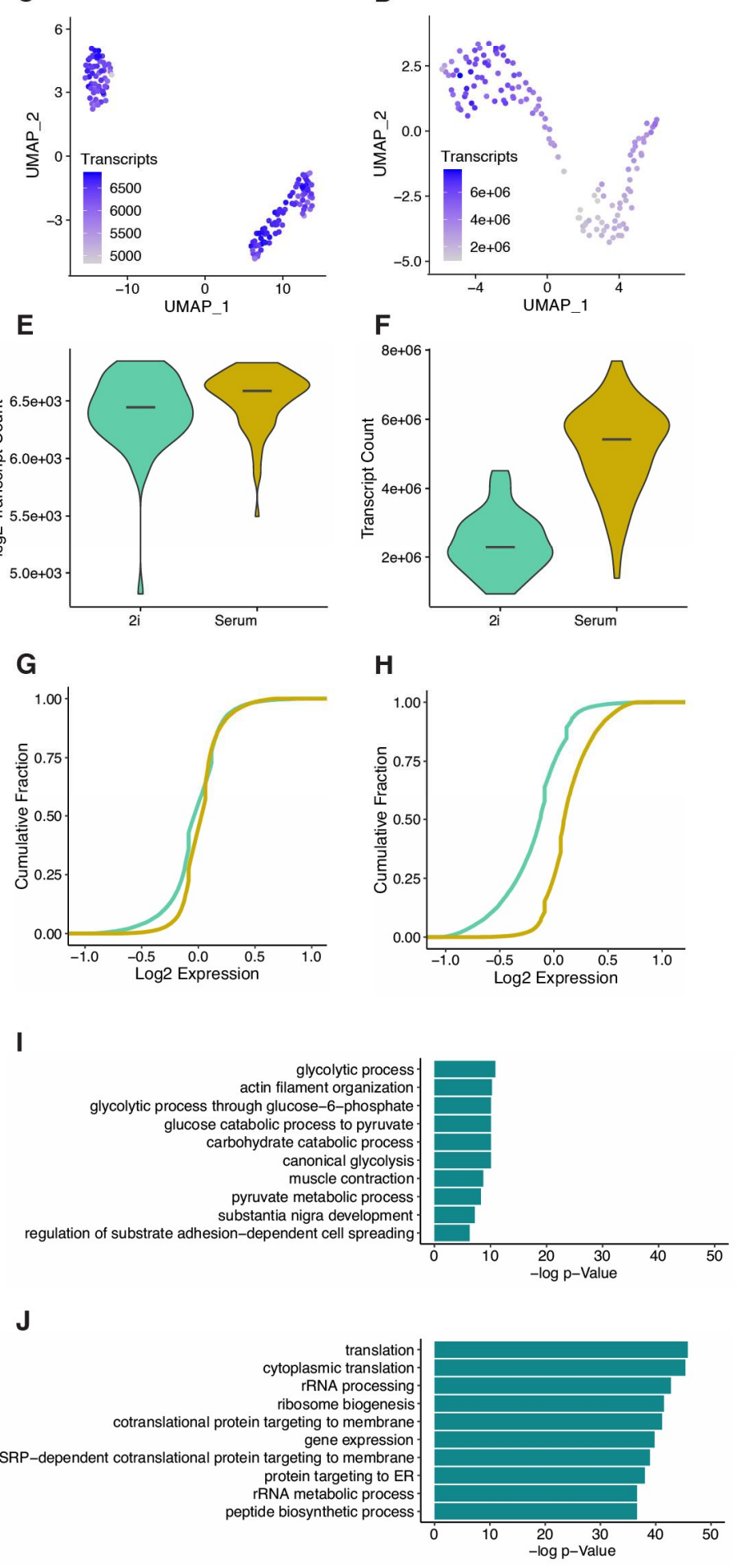
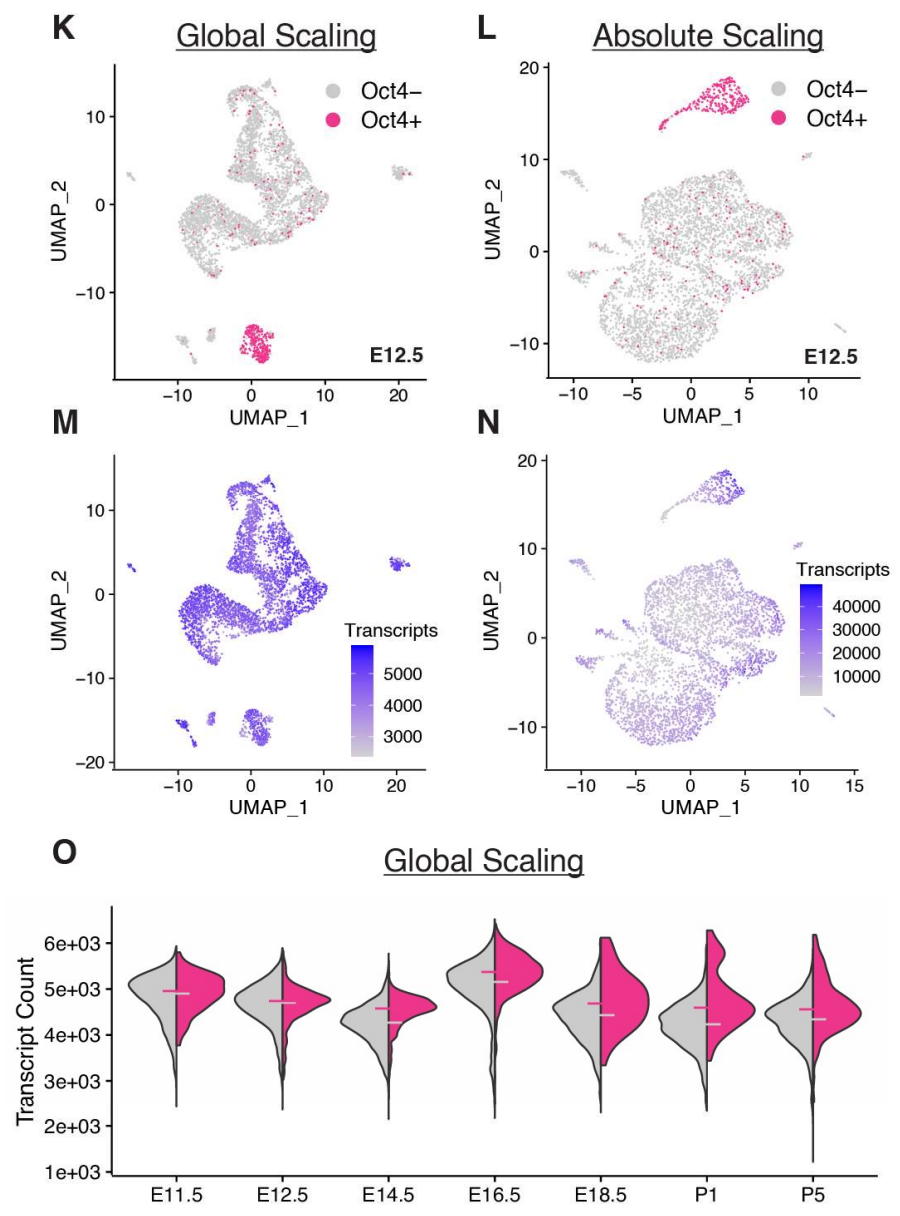

P
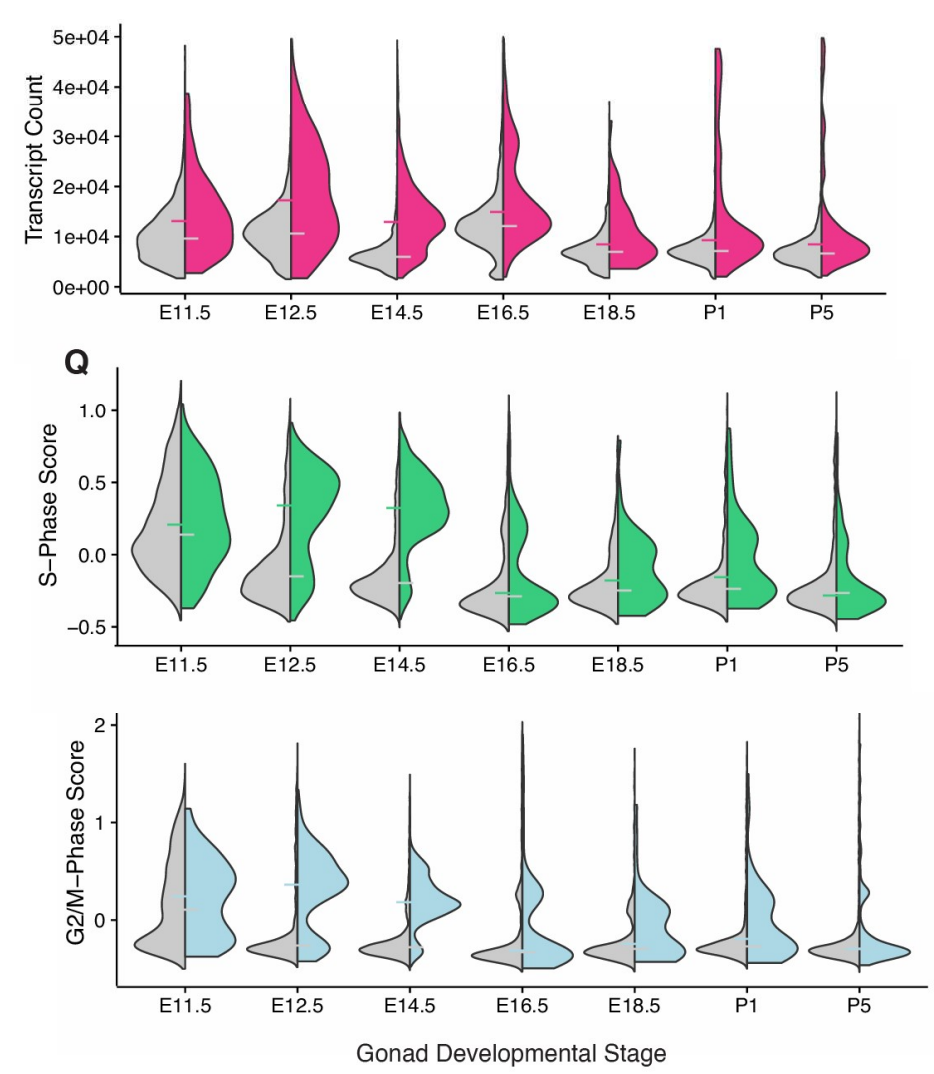
bioRxiv preprint doi: https://doi.org/10.1101/2021.12.13.472426; this version posted December 13, 2021. The copyright holder for this preprint (which was not certified by peer review) is the author/funder. All rights reserved. No reuse allowed without permission.

A

Tabula Muris (Smart-seq2)

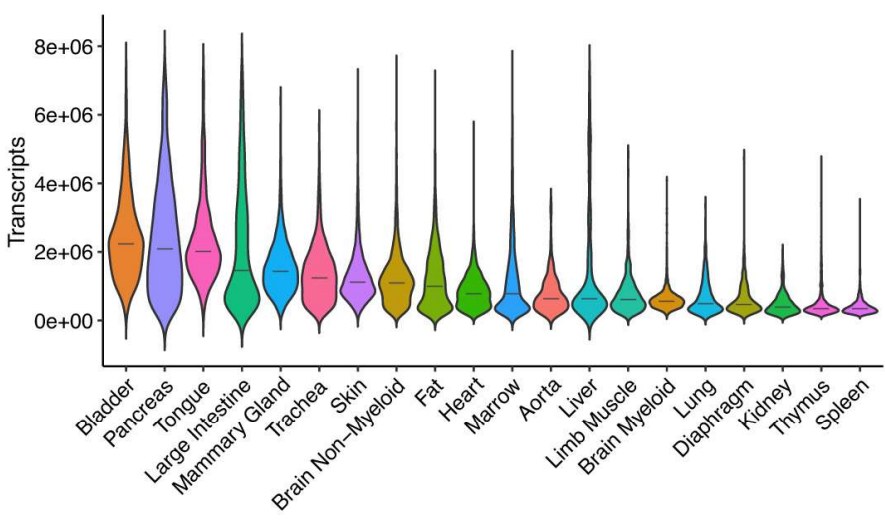

B

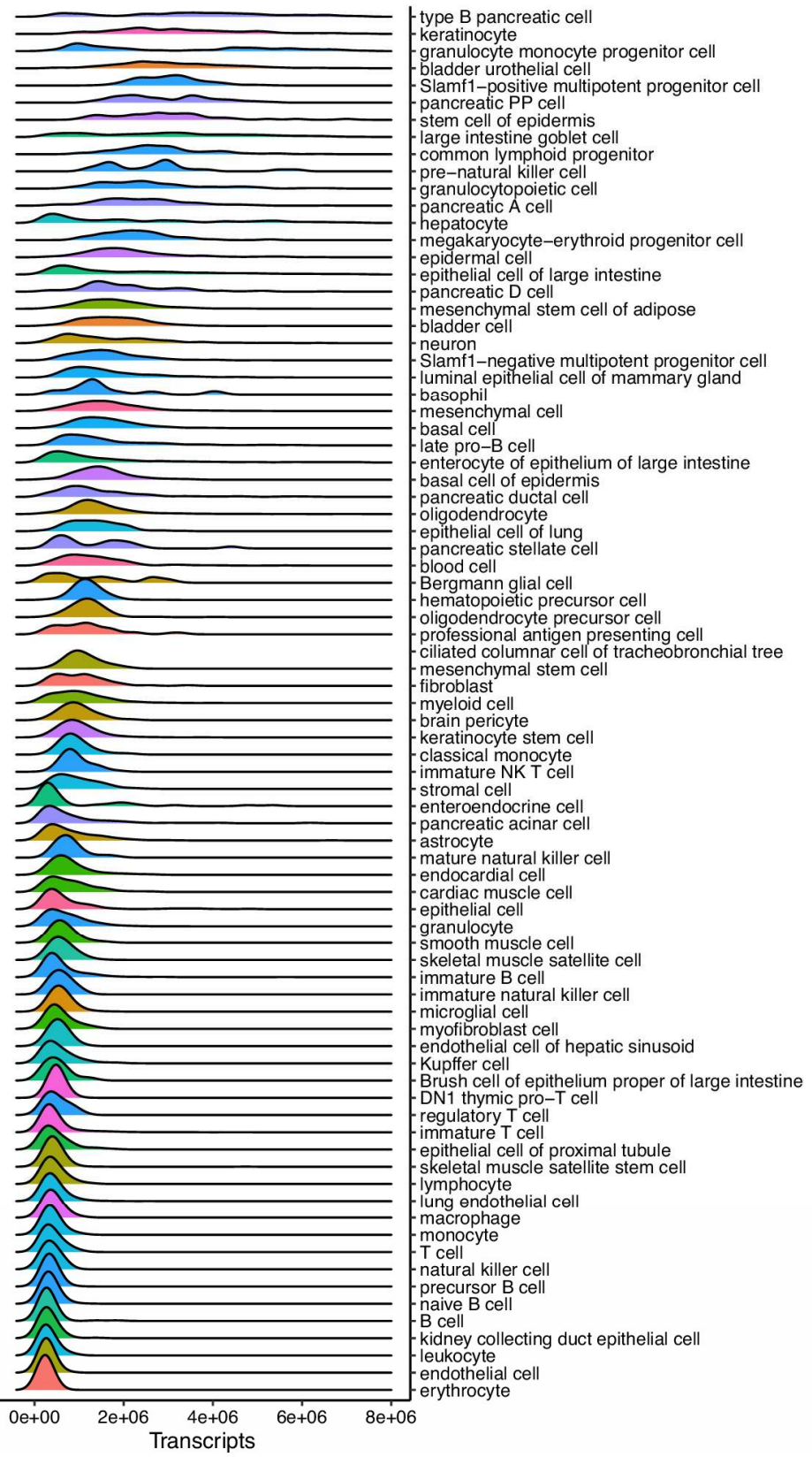

C

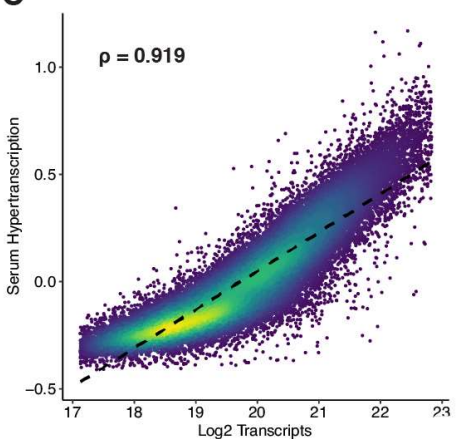

E

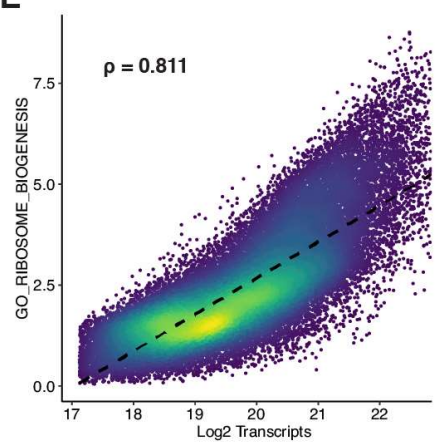

D

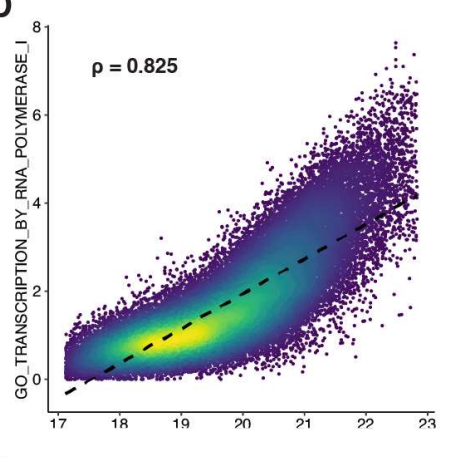

$\mathbf{F}$

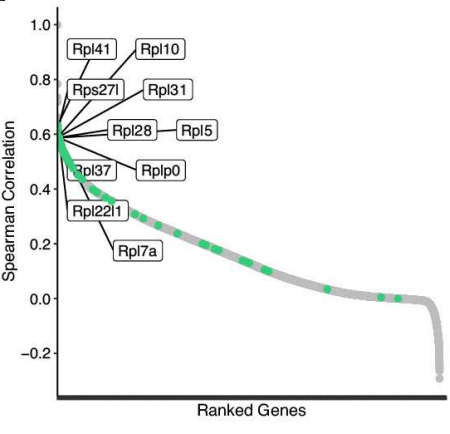

G

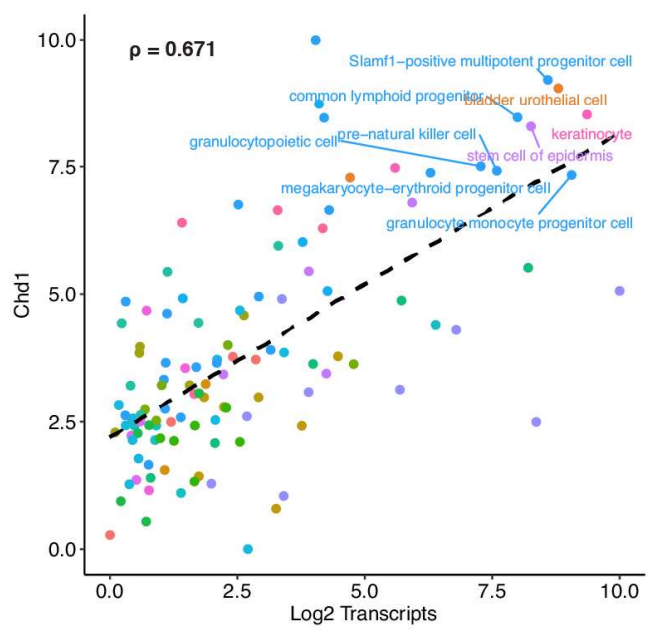

H

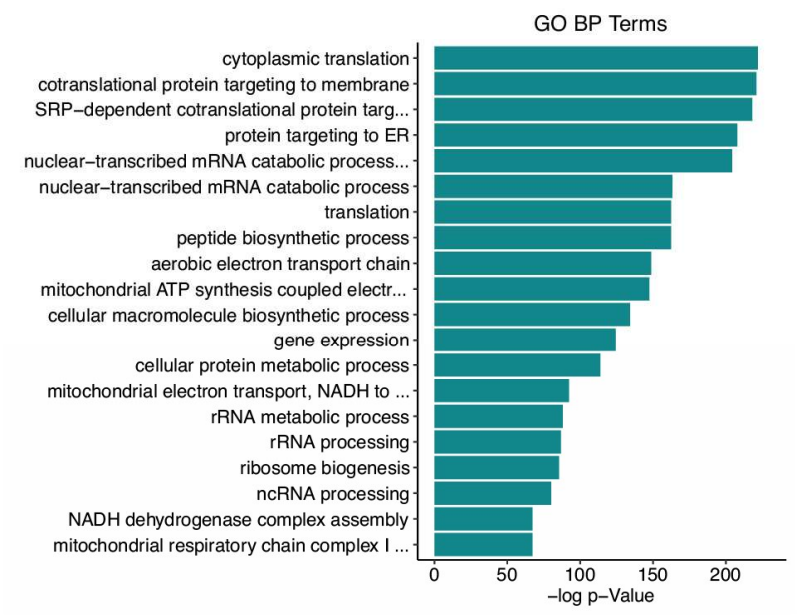


bioRxiv preprint doi: https://doi.org/10.1101/2021.12.13.472426; this version posted December 13, 2021. The copyright holder for this preprint (which was not certified by peer review) is the author/funder. All rights reserved. No reuse allowed without permission.

A

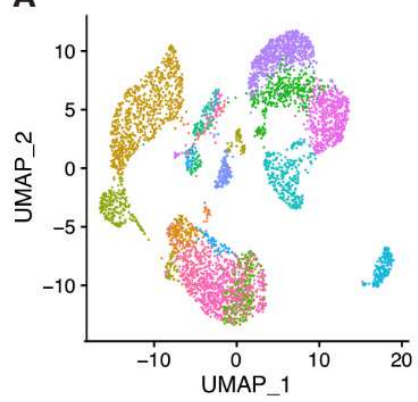

C

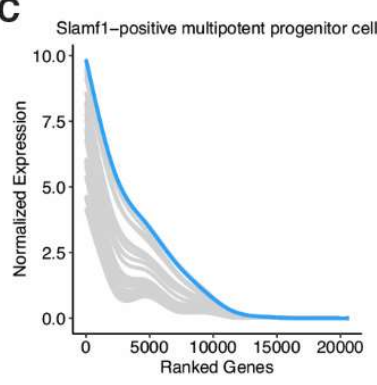

D

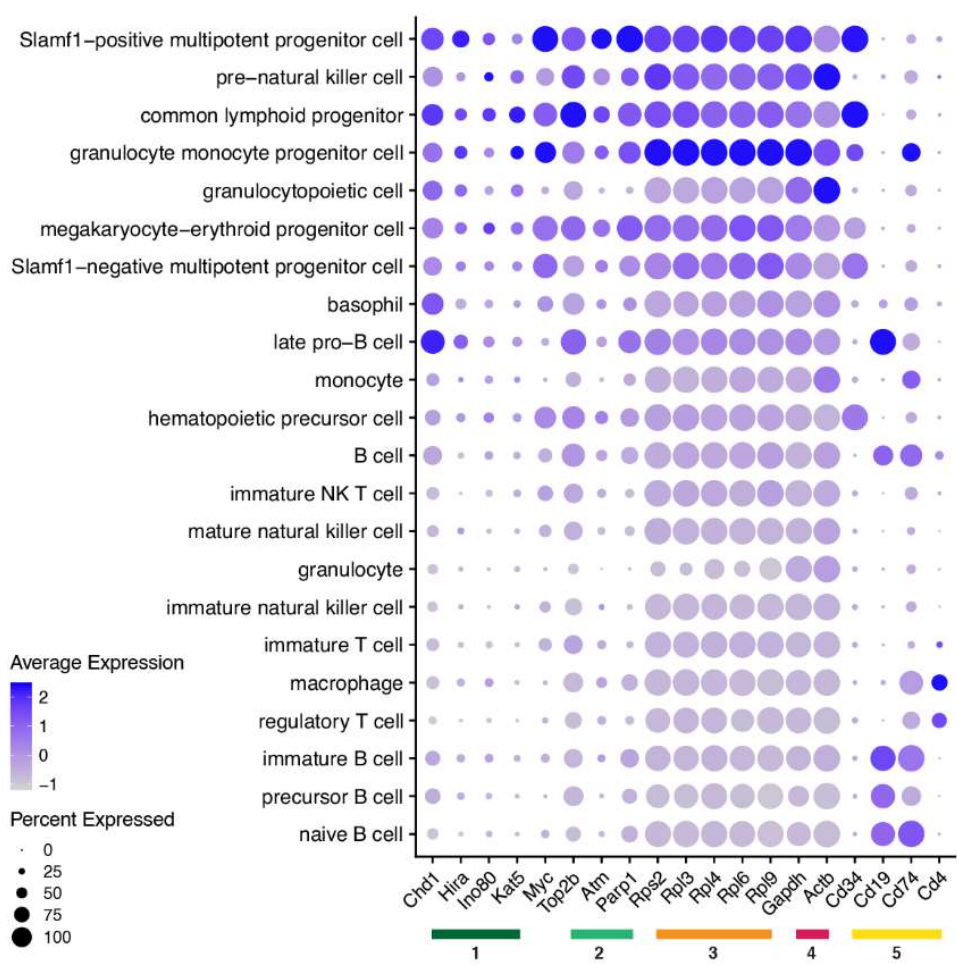

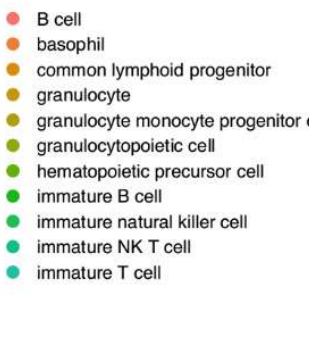

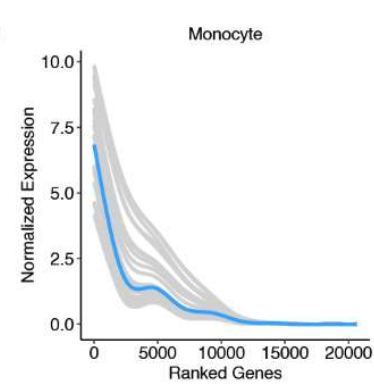

B

- late pro-B cell

- macrophage

mature natural killer cell

- megakaryocyte-erythroid progenitor cell

- monocyte

pre-natural killer cell

- precursor B cell

- regulatory $T$ cell

- Slamf1-negative multipotent progenitor cell

- Slamf1-positive multipotent progenitor cell

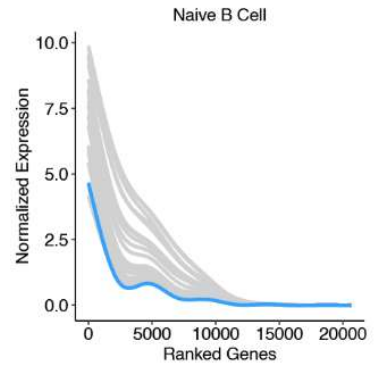

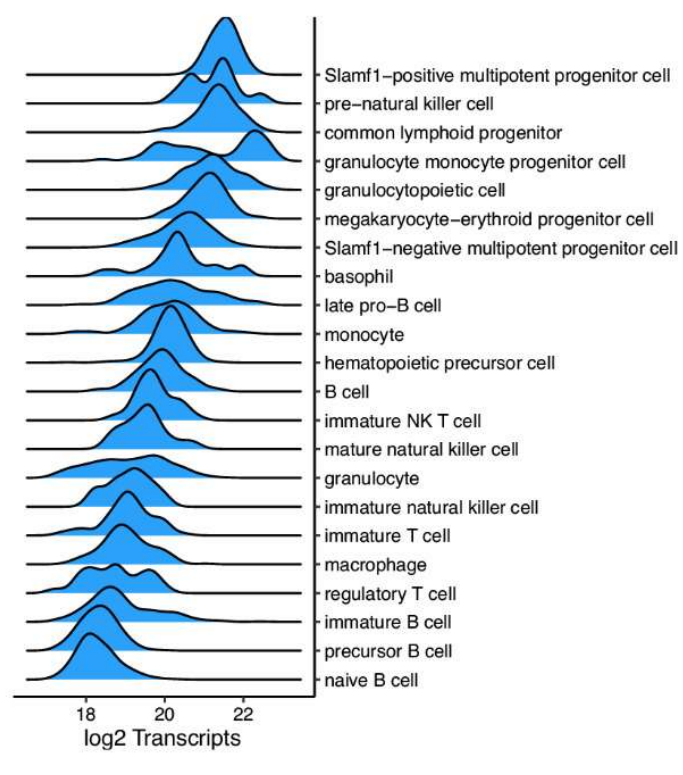

E

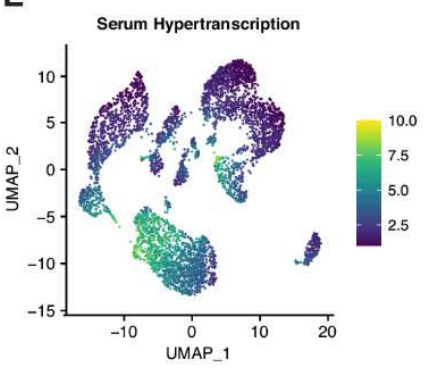

$\mathbf{F}$
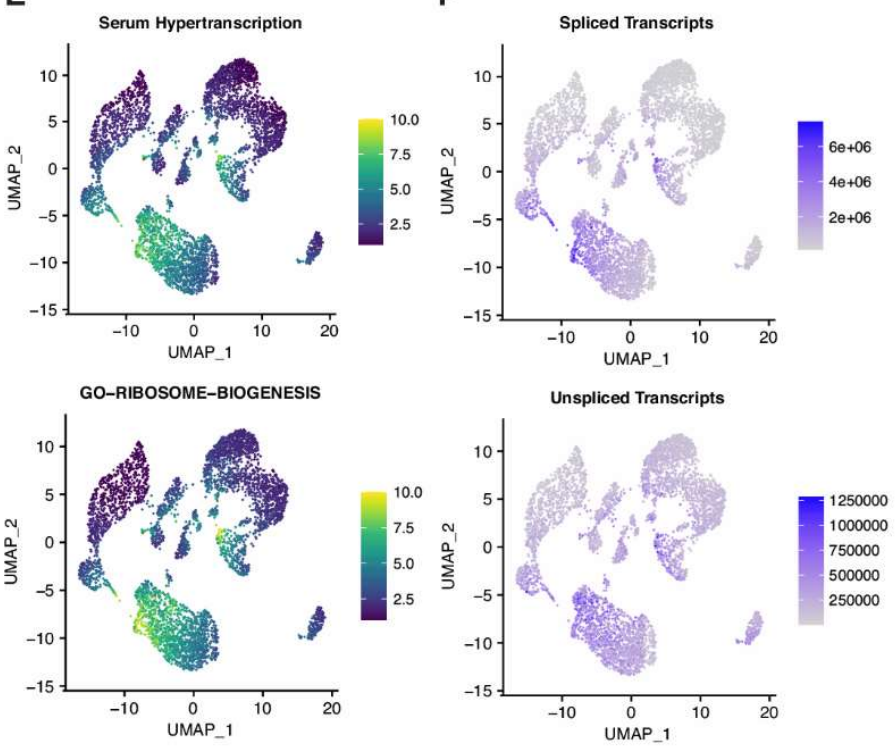

GO-TRANSCRIPTION-BY-RNA-POLYMERASE-I
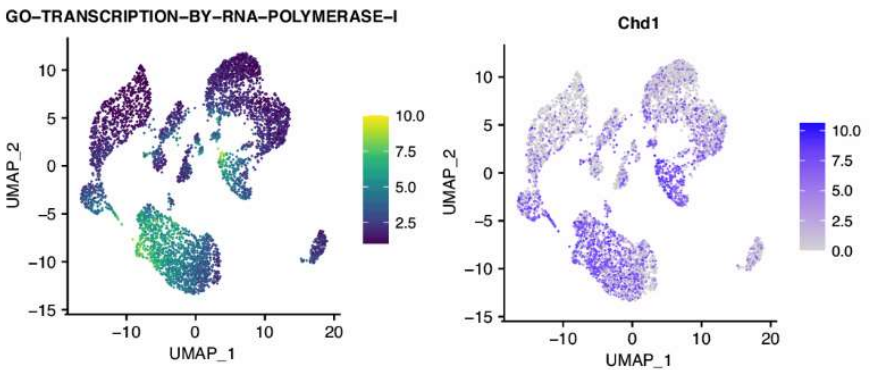
bioRxiv preprint doi: https://doi.org/10.1101/2021.12.13.472426; this version posted December 13, 2021. The copyright holder for this preprint (which was not certified by peer review) is the author/funder. All rights reserved. No reuse allowed without permission.

A

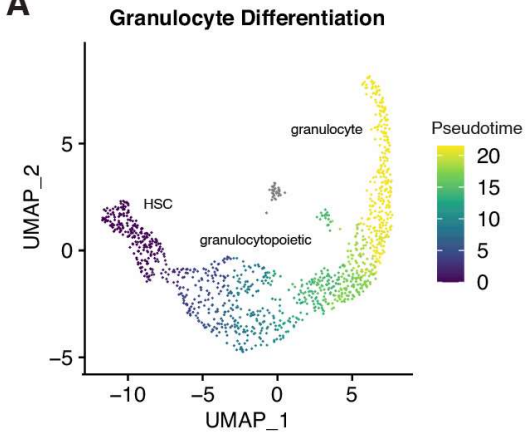

B

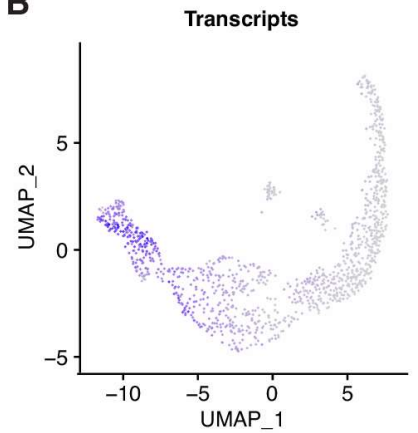

D

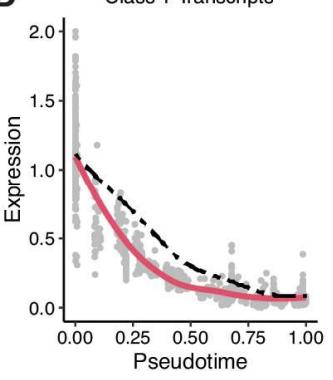

E

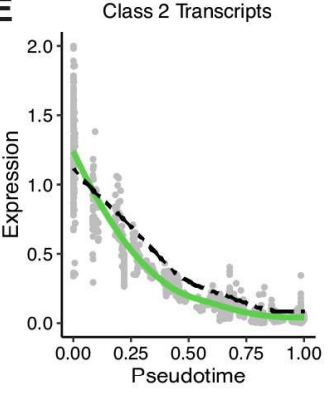

$\mathbf{F}$

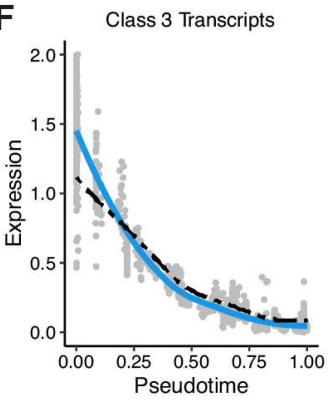

C
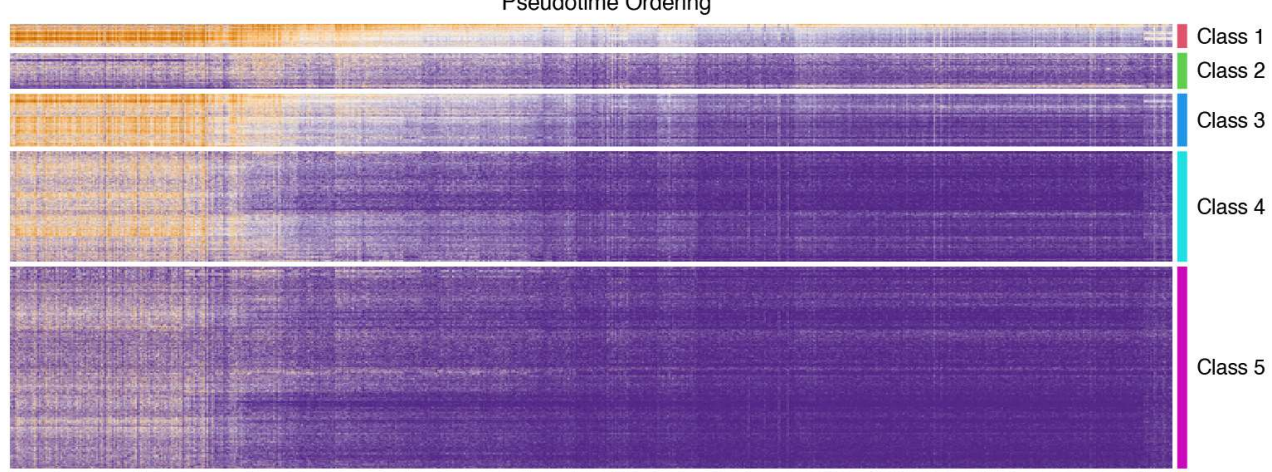

Class 5

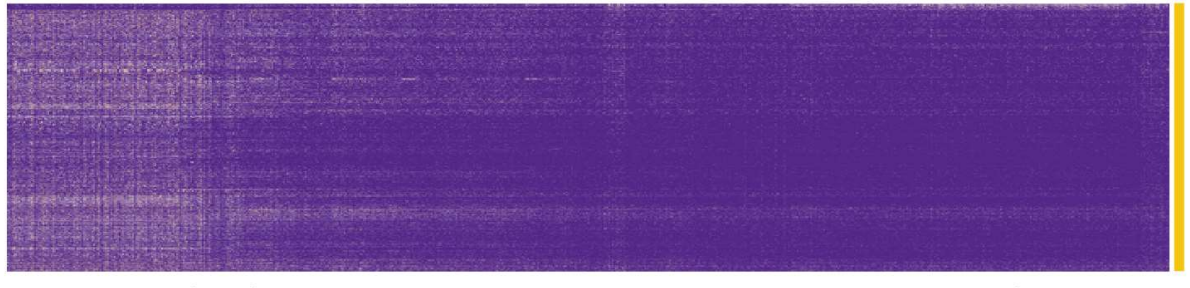

G

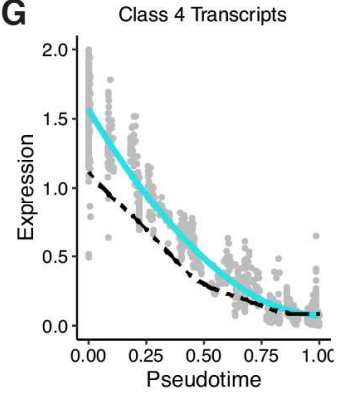

GO BP Terms

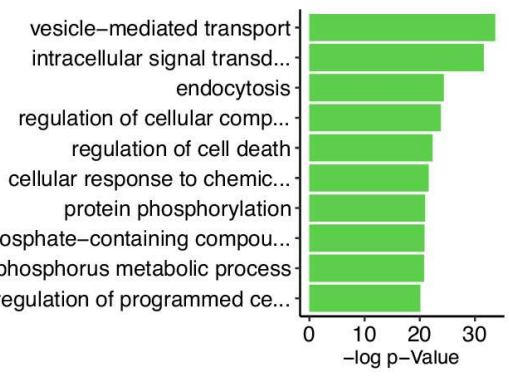

GO BP Terms

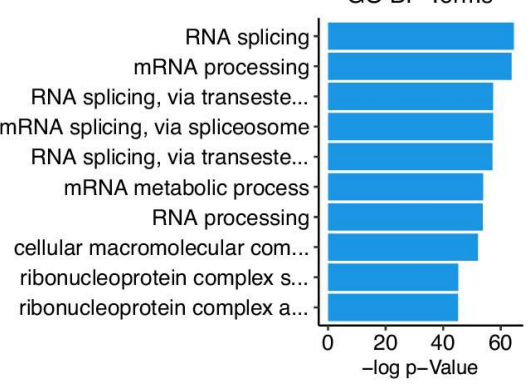
peptide biosynthetic process cellular amide metabolic pr...

amide biosynthetic process organonitrogen compound bio. ribosome biogenesis regulation of actin polymer. regulation of actin filamen. ribosomal small subunit bio...

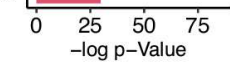

Pseudotime

H

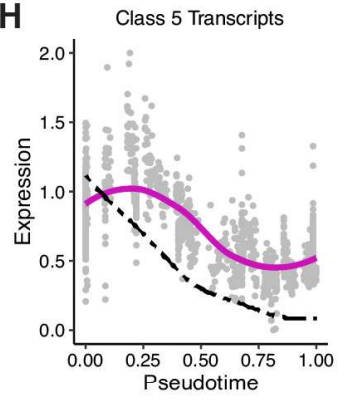

I

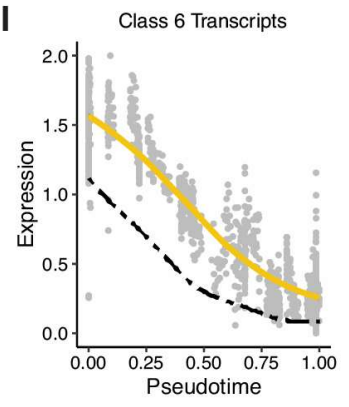

Granulocyte

GO BP Terms

RNA processing mRNA metabolic process ribonucleoprotein complex b... mRNA processing cellular macromolecule cata... cellular catabolic process RNA splicing macromolecular complex subu... gene expression macromolecule catabolic pro.

Class 6
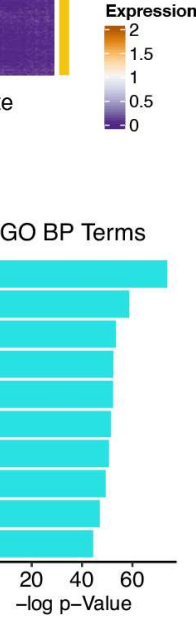

GO BP Terms

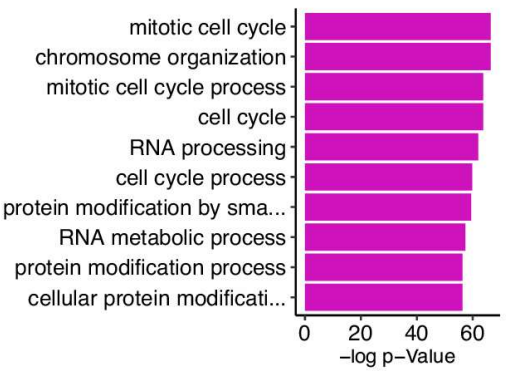

GO BP Terms

RNA metabolic process cellular macromolecule bios... gene expression heterocycle biosynthetic pr... regulation of macromolecule... aromatic compound biosynthe... regulation of nitrogen comp.

regulation of cellular macr. regulation of nucleobase-co nucleobase-containing compo.

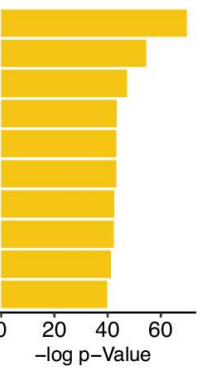


bioRxiv preprint doi: https://doi.org/10.1101/2021.12.13.472426; this version posted December 13, 2021. The copyright holder for this preprint (which was not certified by peer review) is the author/funder. All rights reserved. No reuse allowed without permission.

A

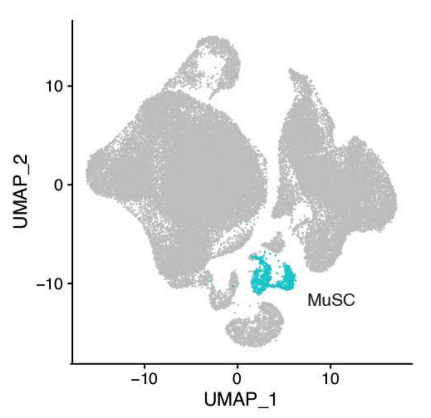

B

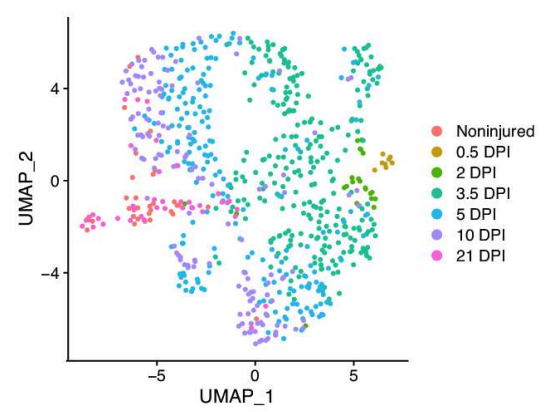

C

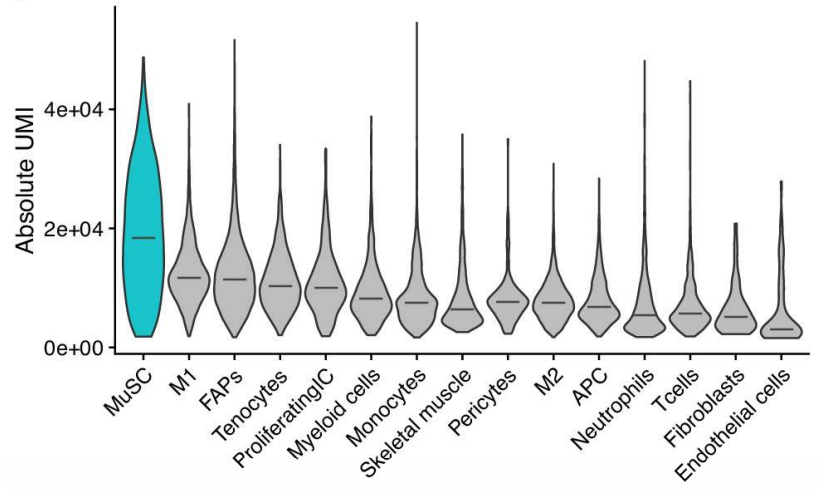

E
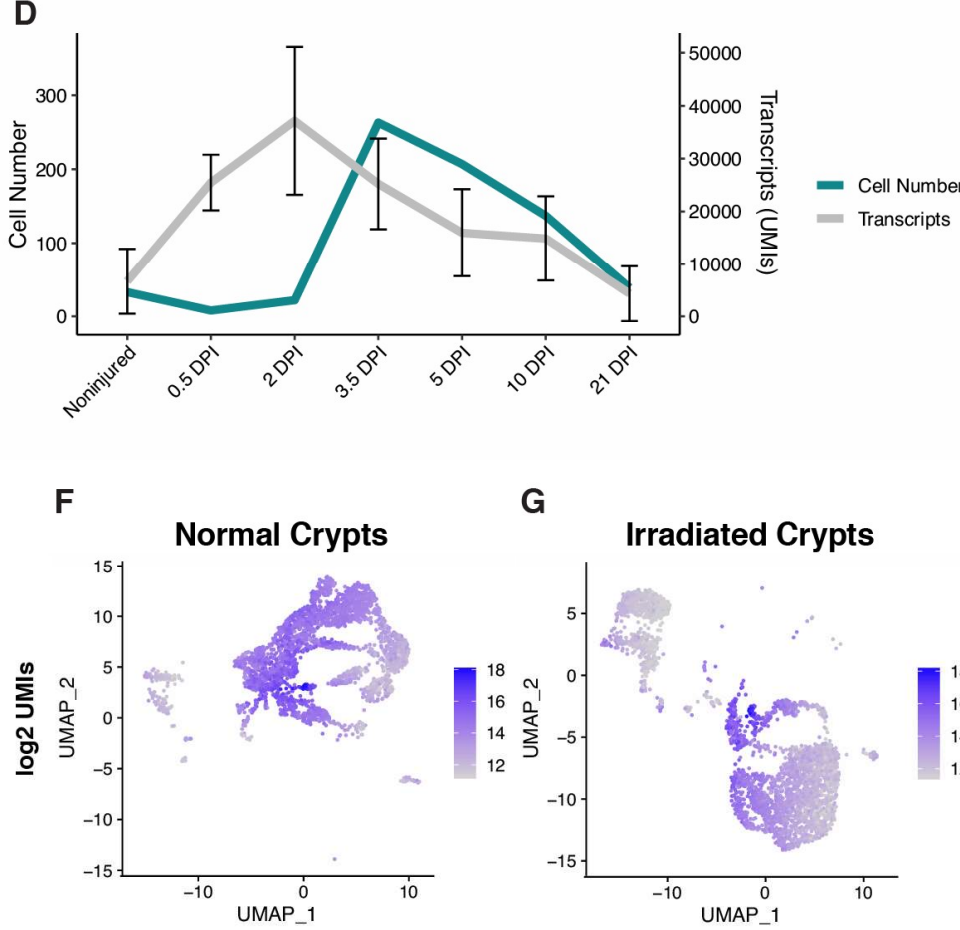

G
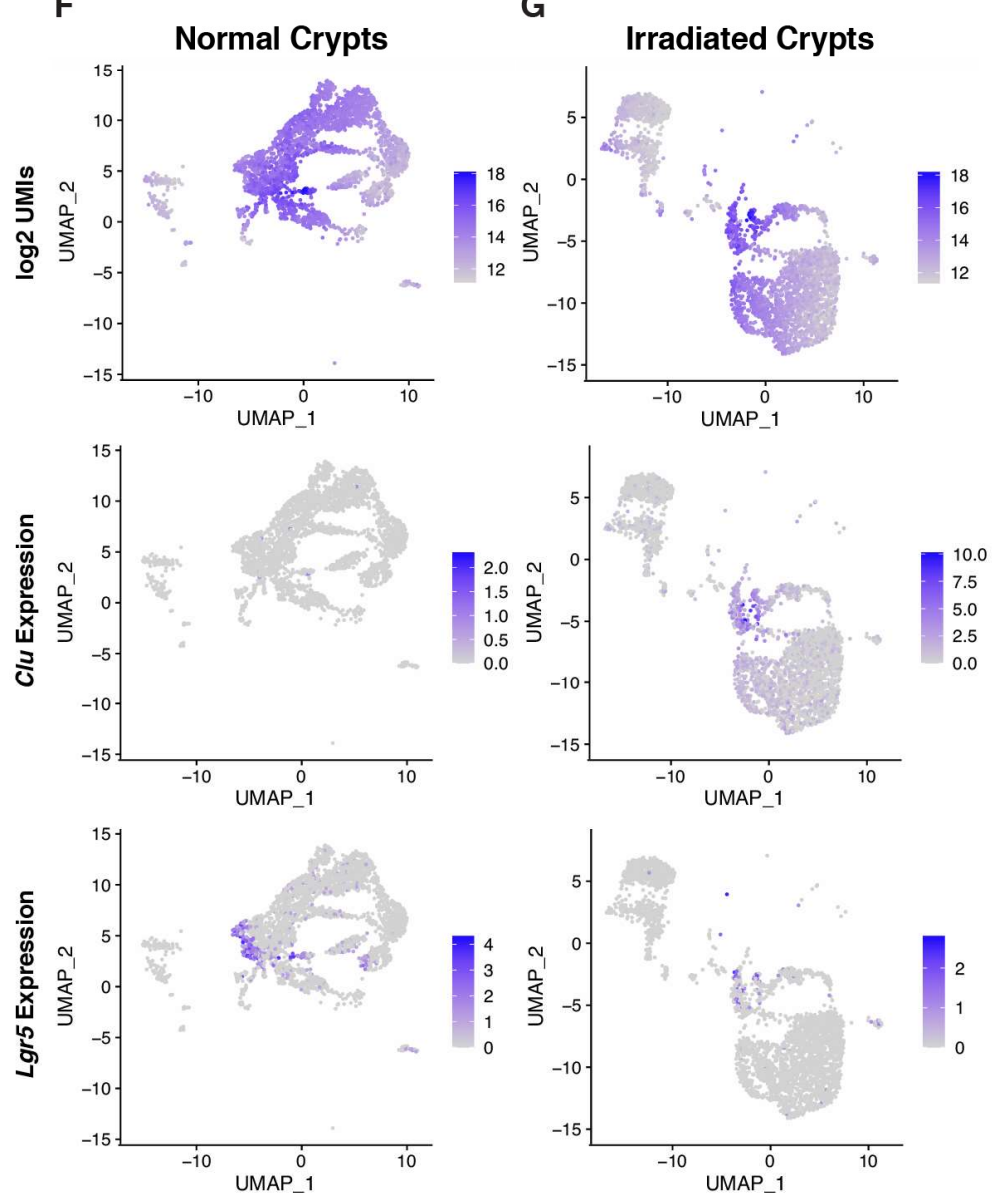

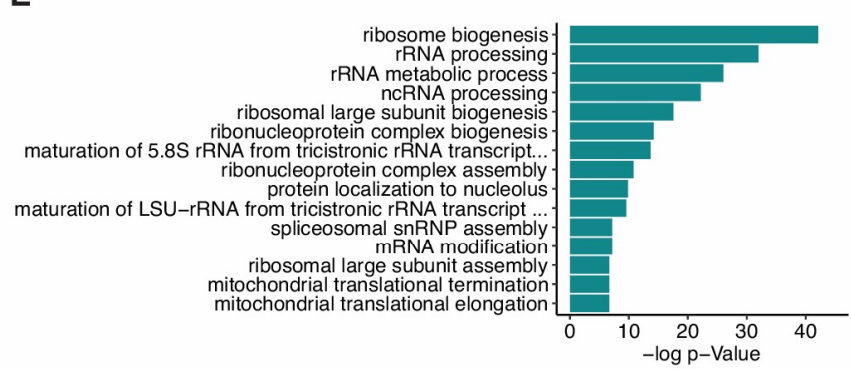

H
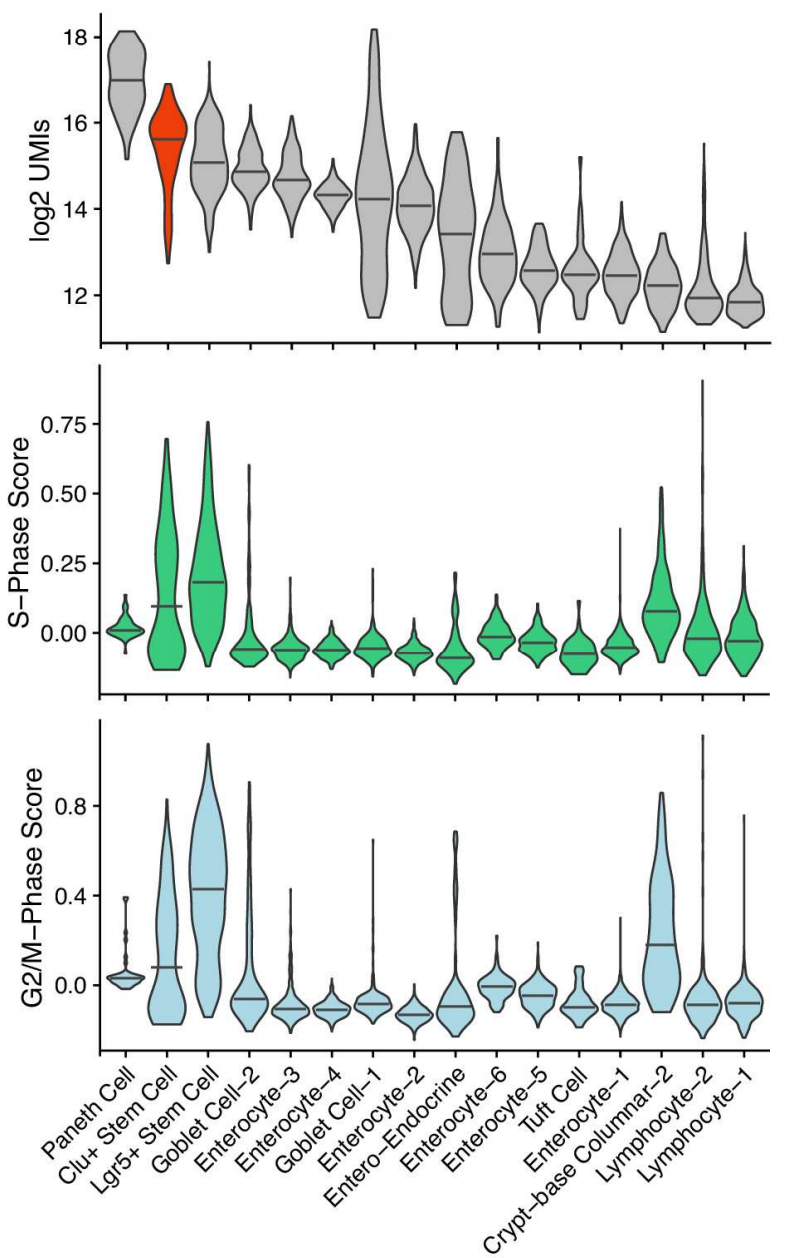
bioRxiv preprint doi: https://doi.org/10.1101/2021.12.13.472426; this version posted December 13, 2021. The copyright holder for this preprint (which was not certified by peer review) is the author/funder. All rights reserved. No reuse allowed without permission.

A

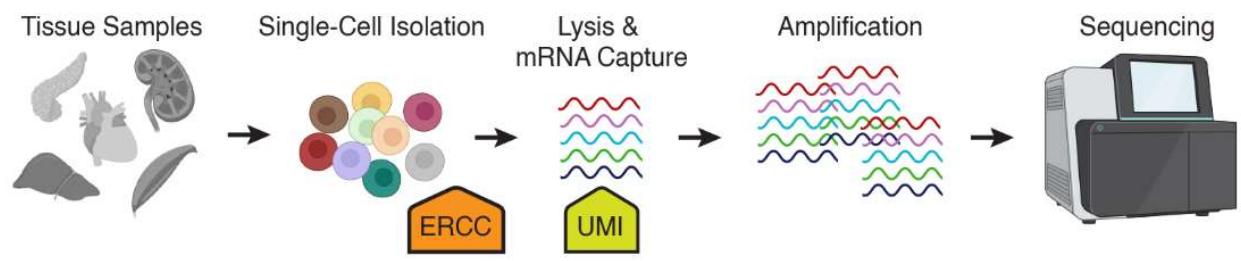

B

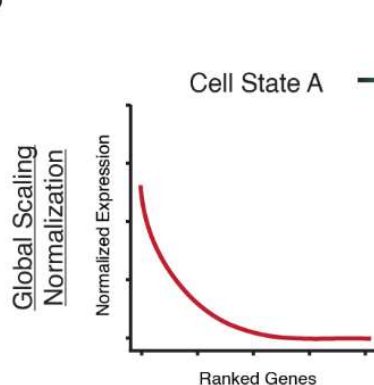

Hypertranscription
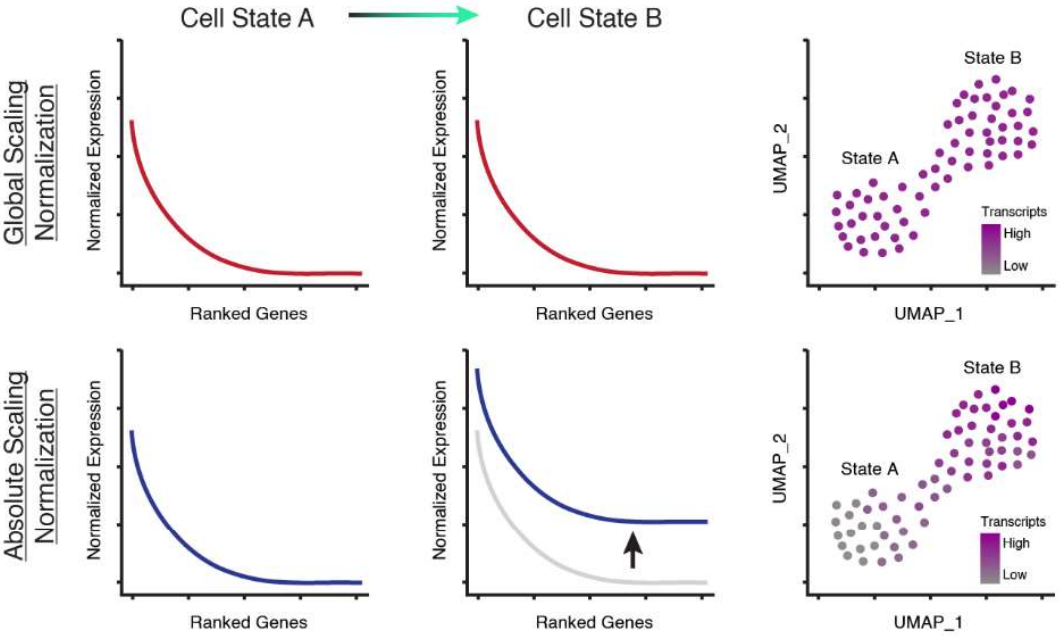

Ranked Genes
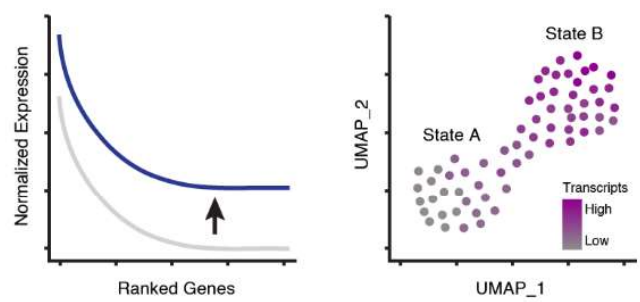

UMAP_1

C

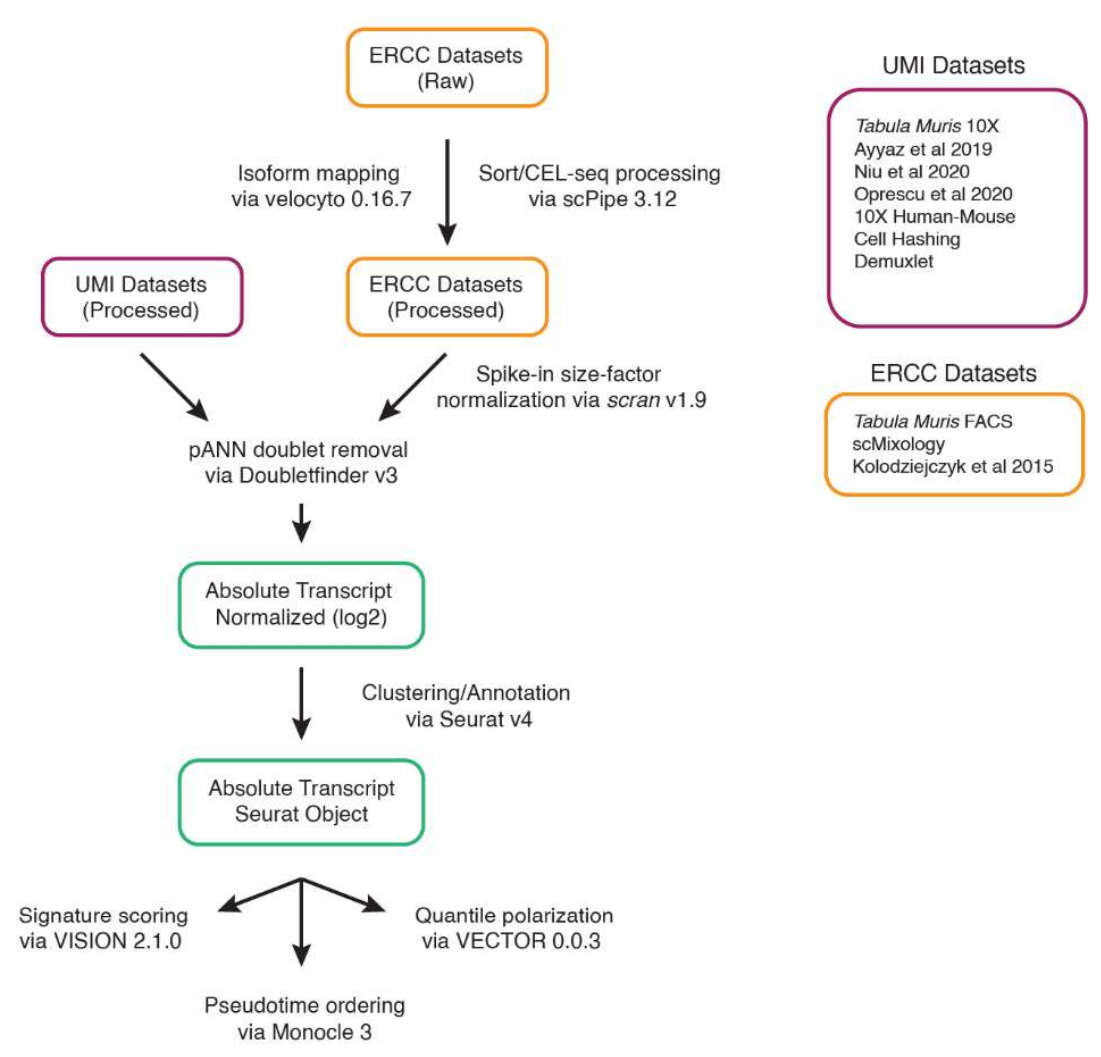

\section{Supplementary Figure 1}


bioRxiv preprint doi: https://doi.org/10.1101/2021.12.13.472426; this version posted December 13, 2021. The copyright holder for this preprint (which was not certified by peer review) is the author/funder. All rights reserved. No reuse allowed without permission.

A
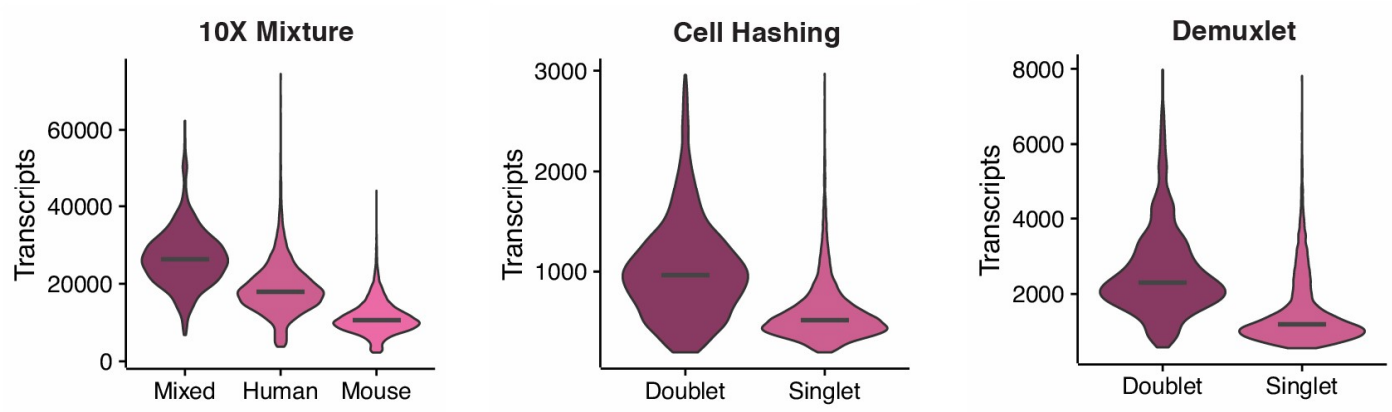

B

C
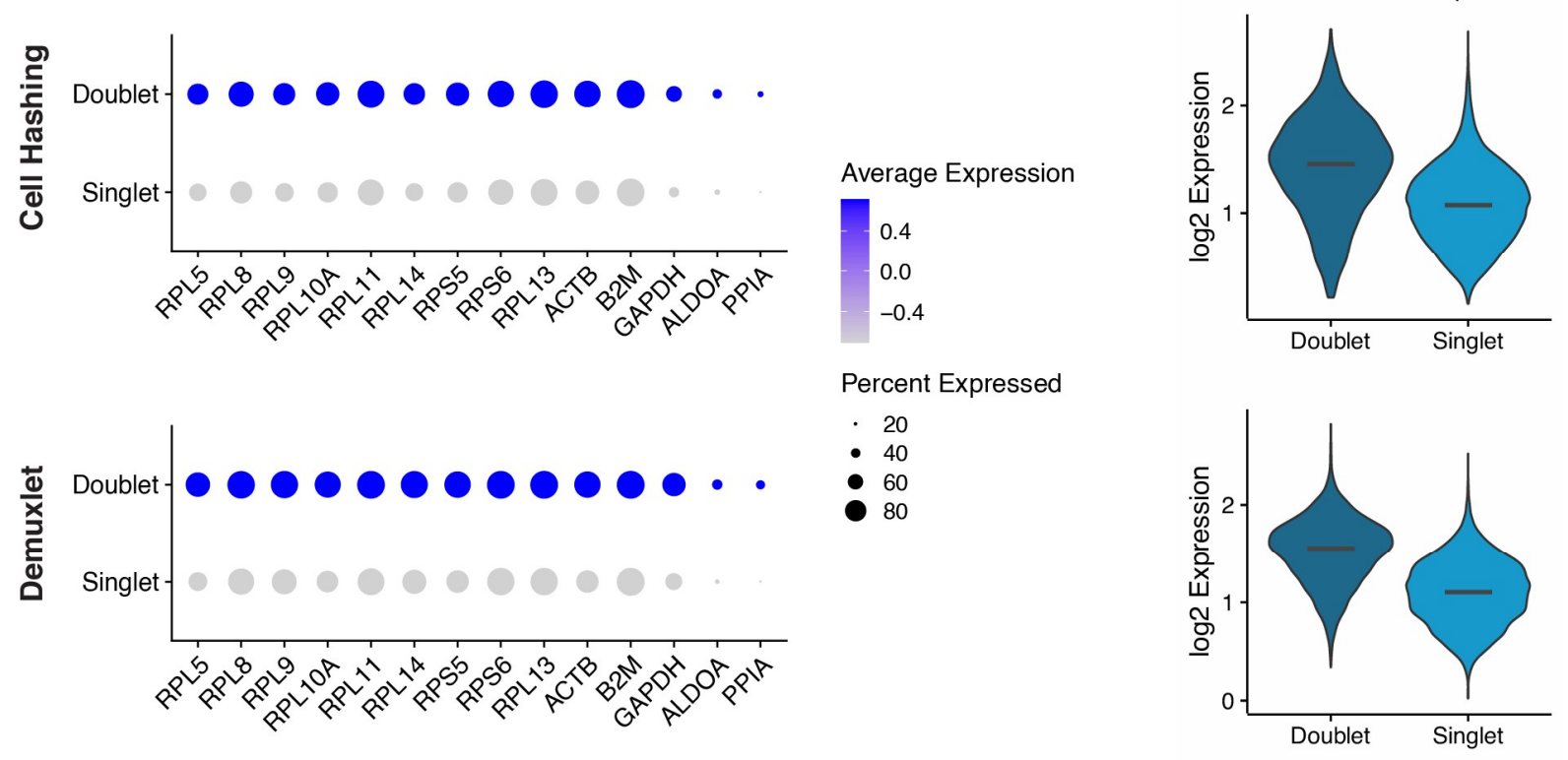
bioRxiv preprint doi: https://doi.org/10.1101/2021.12.13.472426; this version posted December 13, 2021. The copyright holder for this preprint (which was not certified by peer review) is the author/funder. All rights reserved. No reuse allowed without permission.

\section{Sort-seq Mix}

A
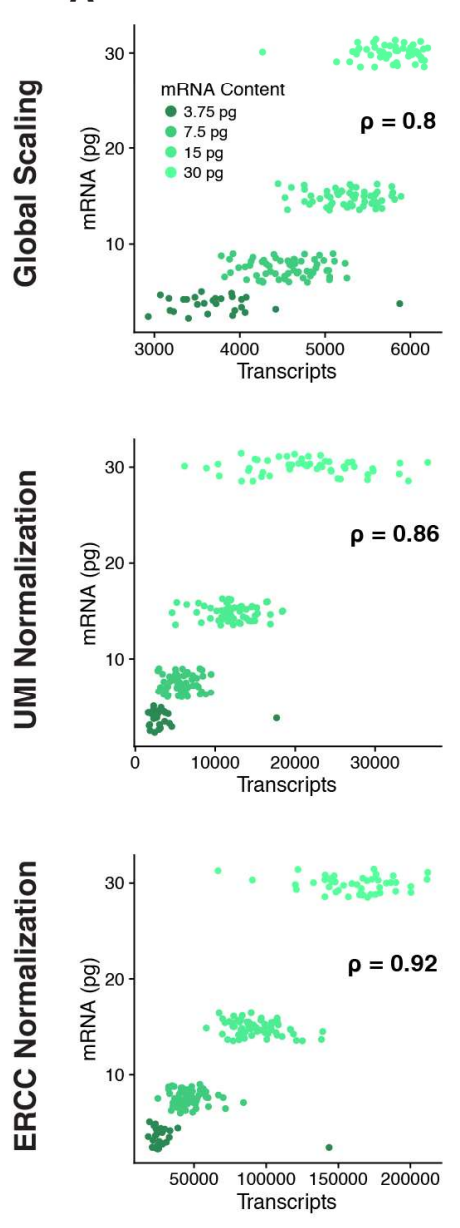

\section{CEL-seq Mix}

E

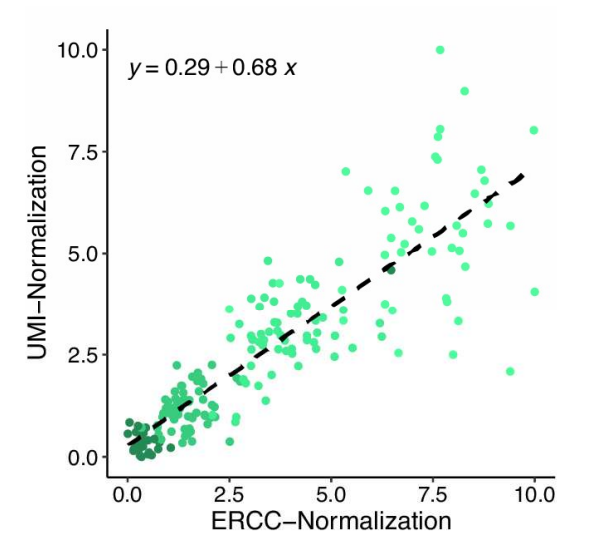

B
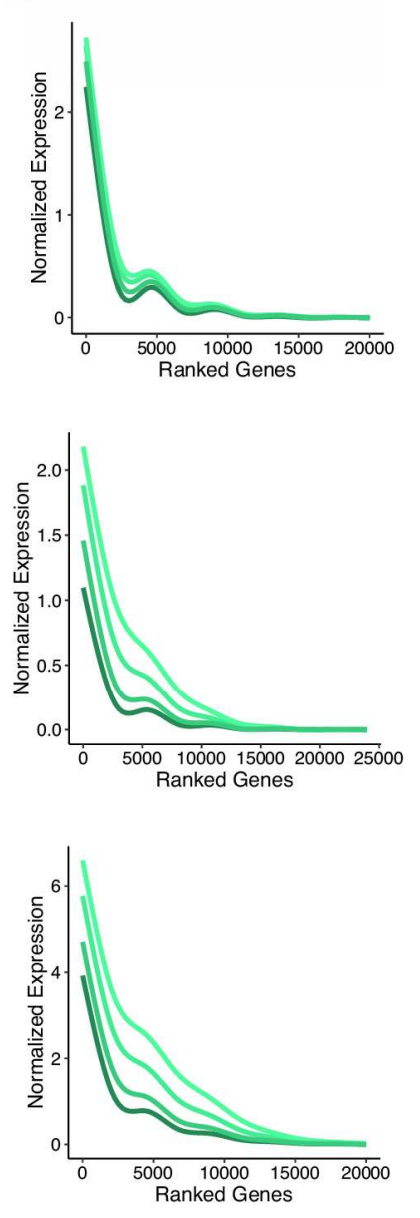

E
C
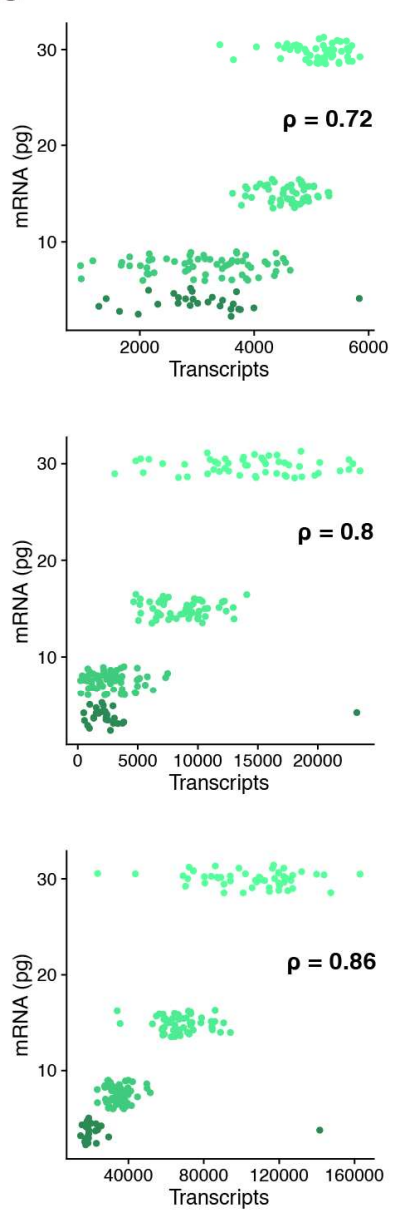

F

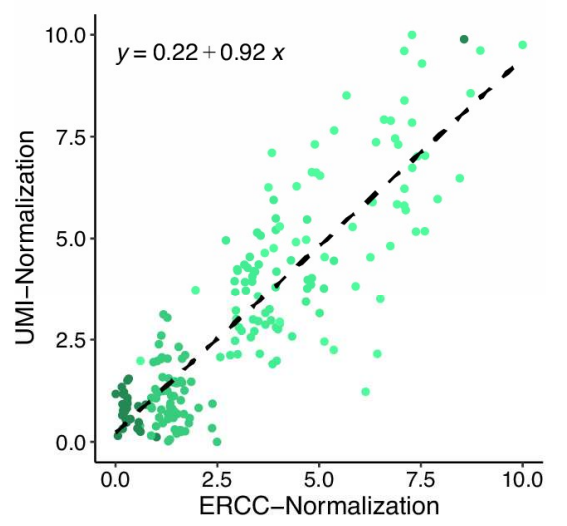

\section{Supplementary Figure 3}


A

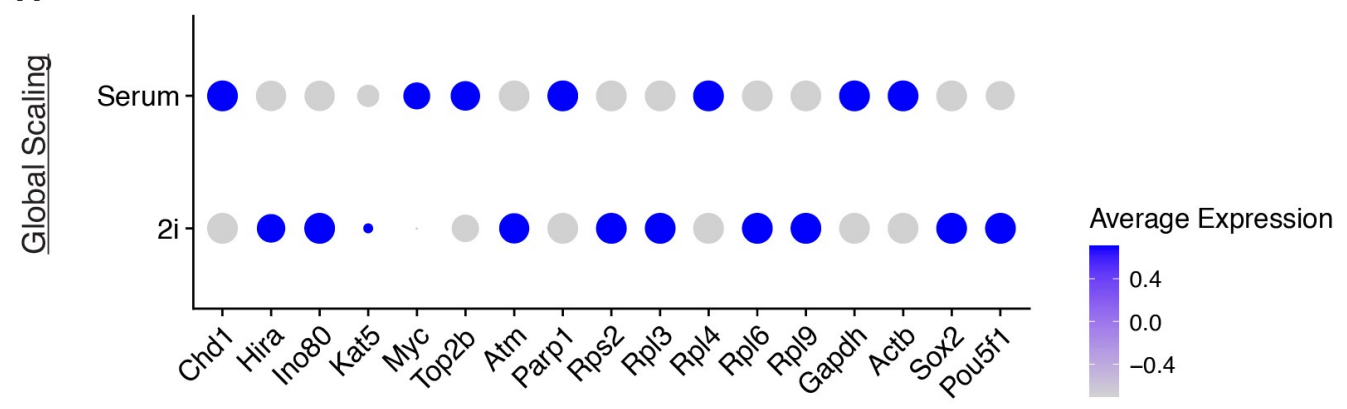

B

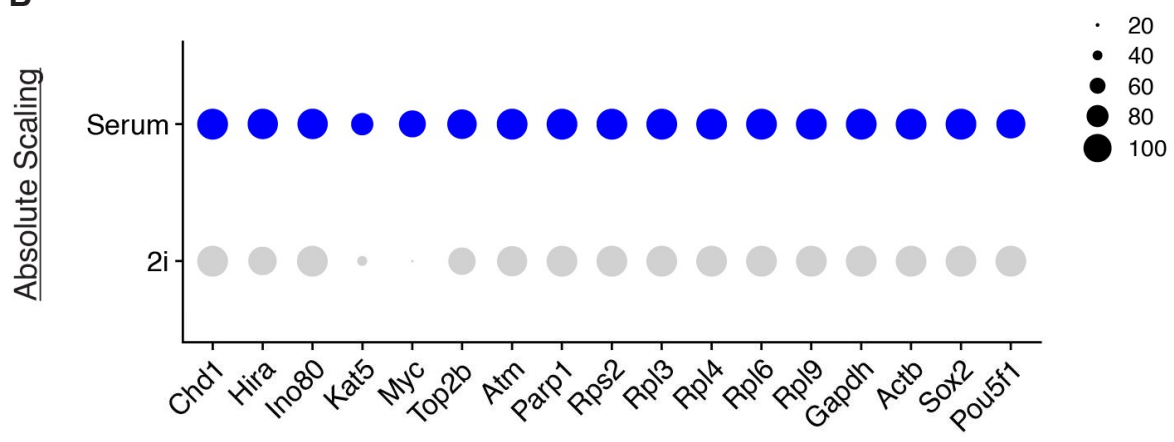

C

D
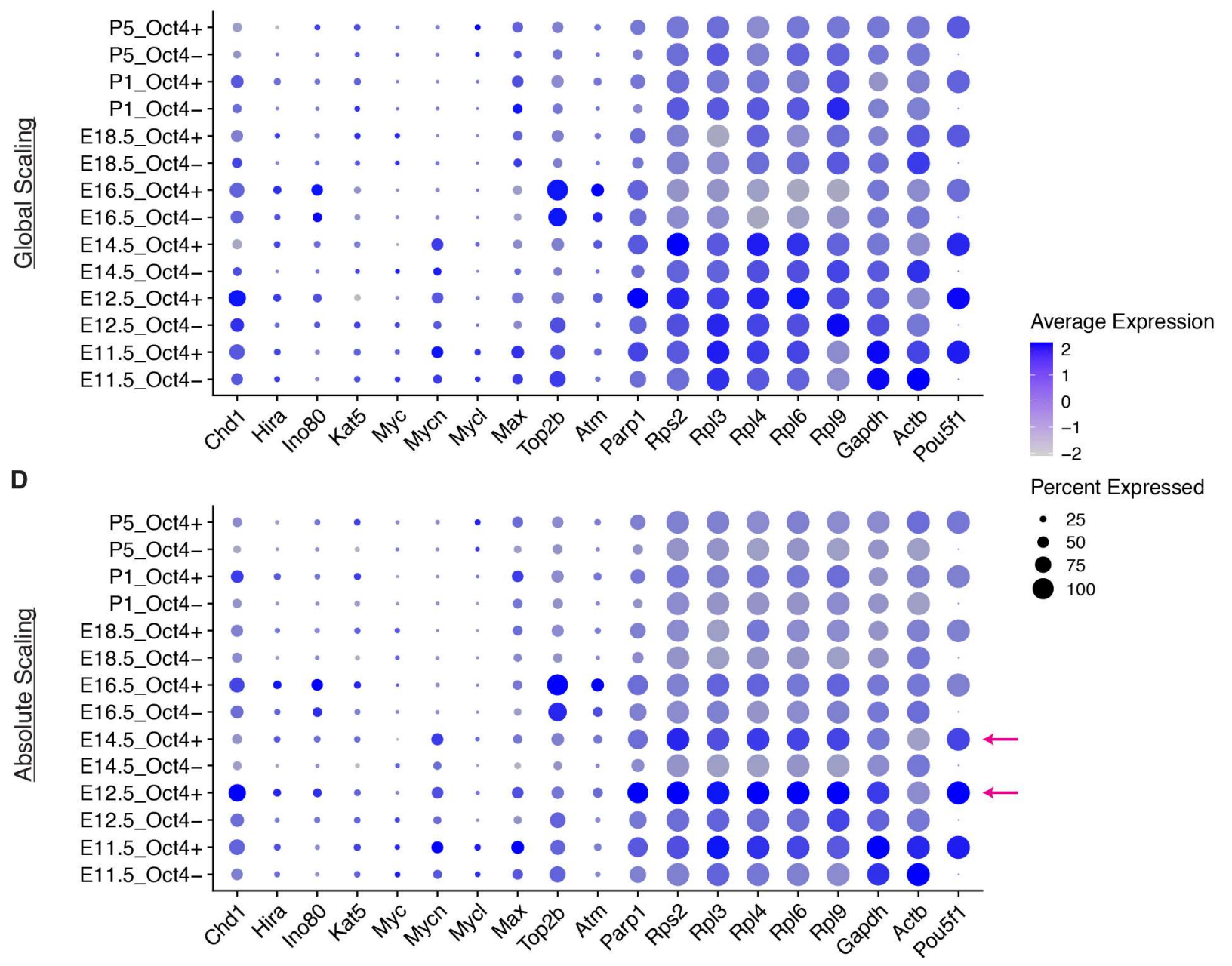
bioRxiv preprint doi: https://doi.org/10.1101/2021.12.13.472426; this version posted December 13,2021 . The copyright holder for this preprint (which was not certified by peer review) is the author/funder. All rights reserved. No reuse allowed without permission.

A

Tabula Muris (10X)

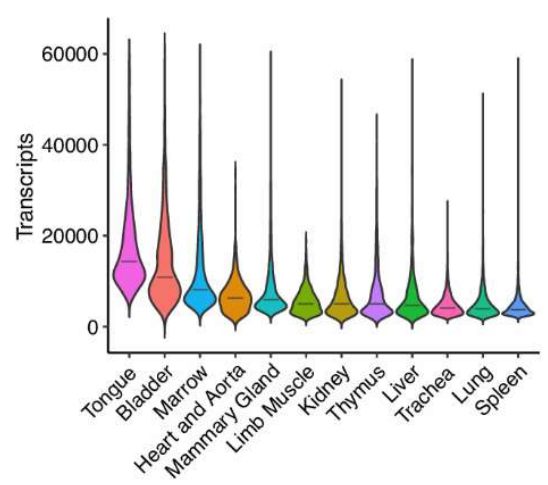

B

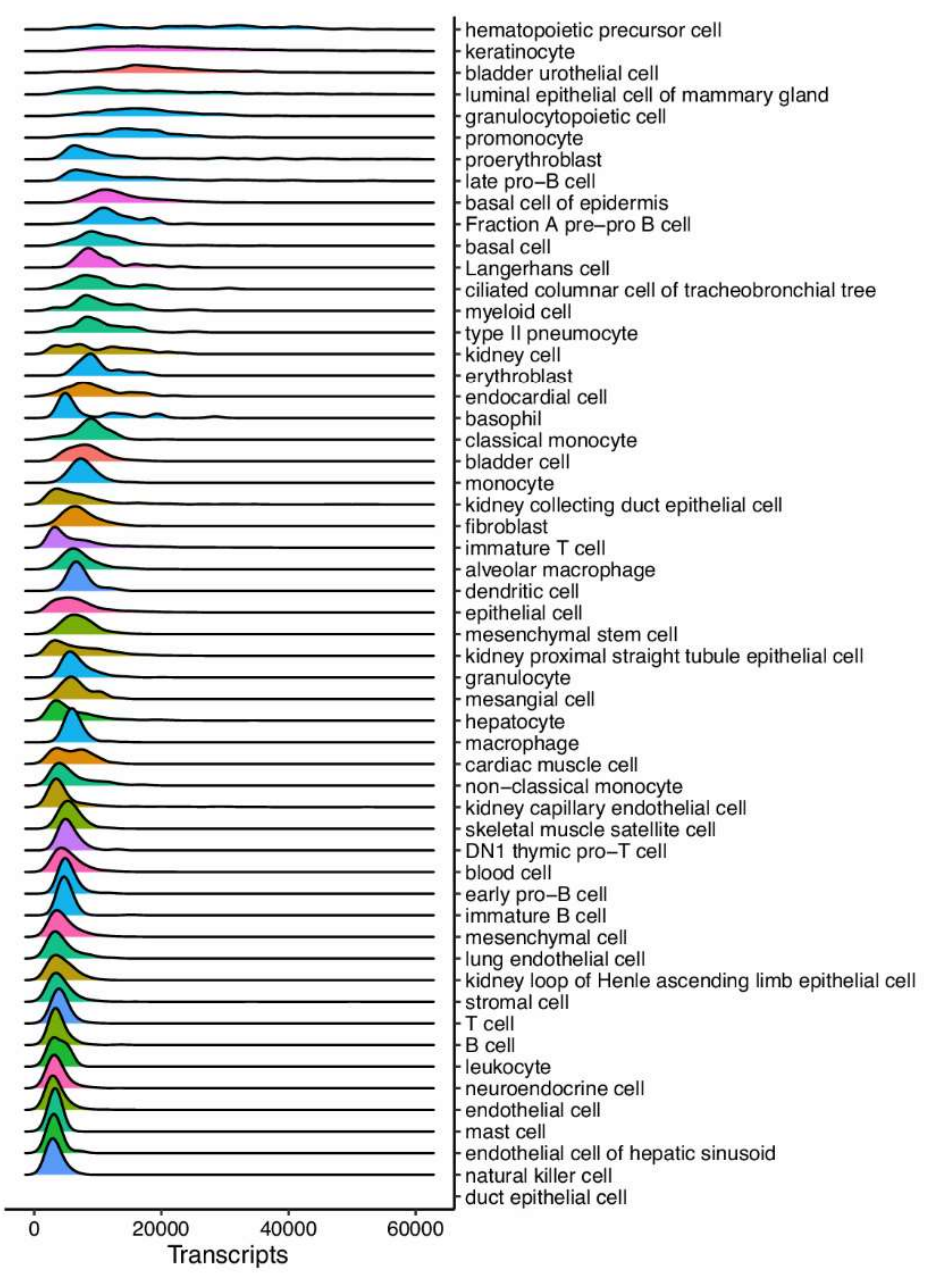

C
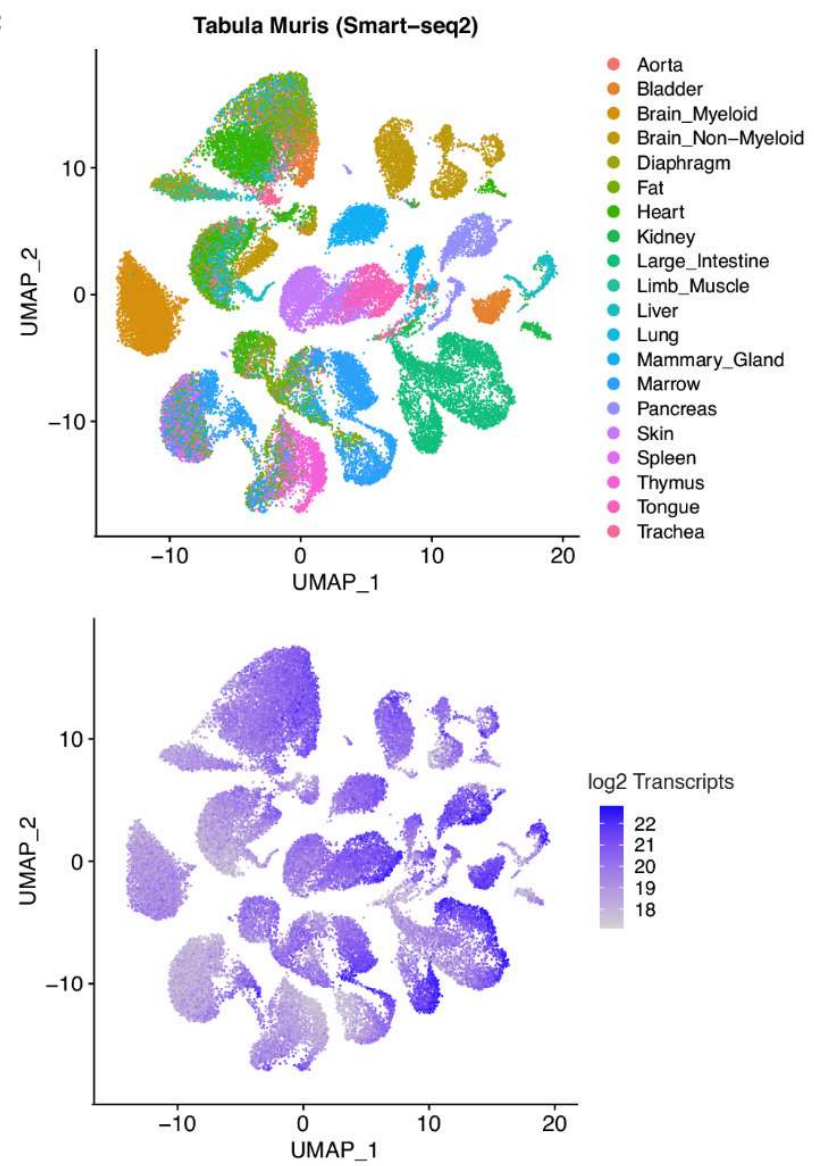

D
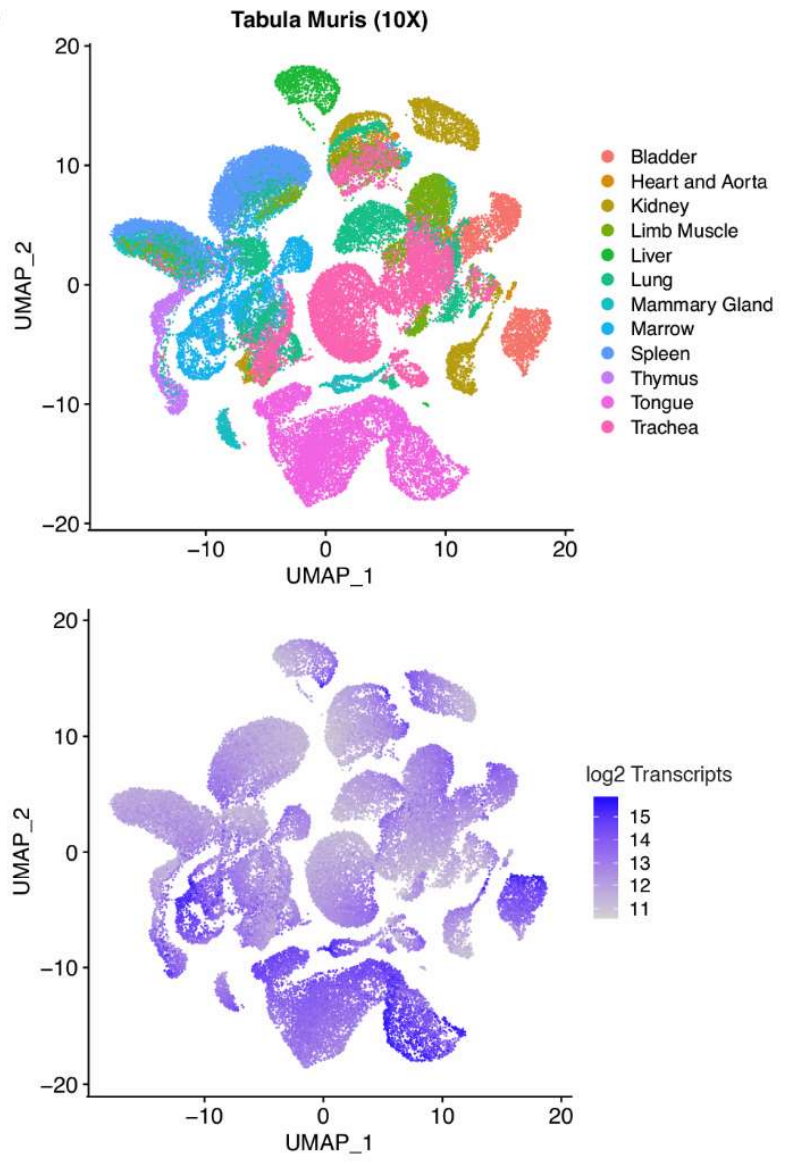

\section{Supplementary Figure 5}


bioRxiv preprint doi: https://doi.org/10.1101/2021.12.13.472426; this version posted December 13, 2021. The copyright holder for this preprint (which was not certified by peer review) is the author/funder. All rights reserved. No reuse allowed without permission.
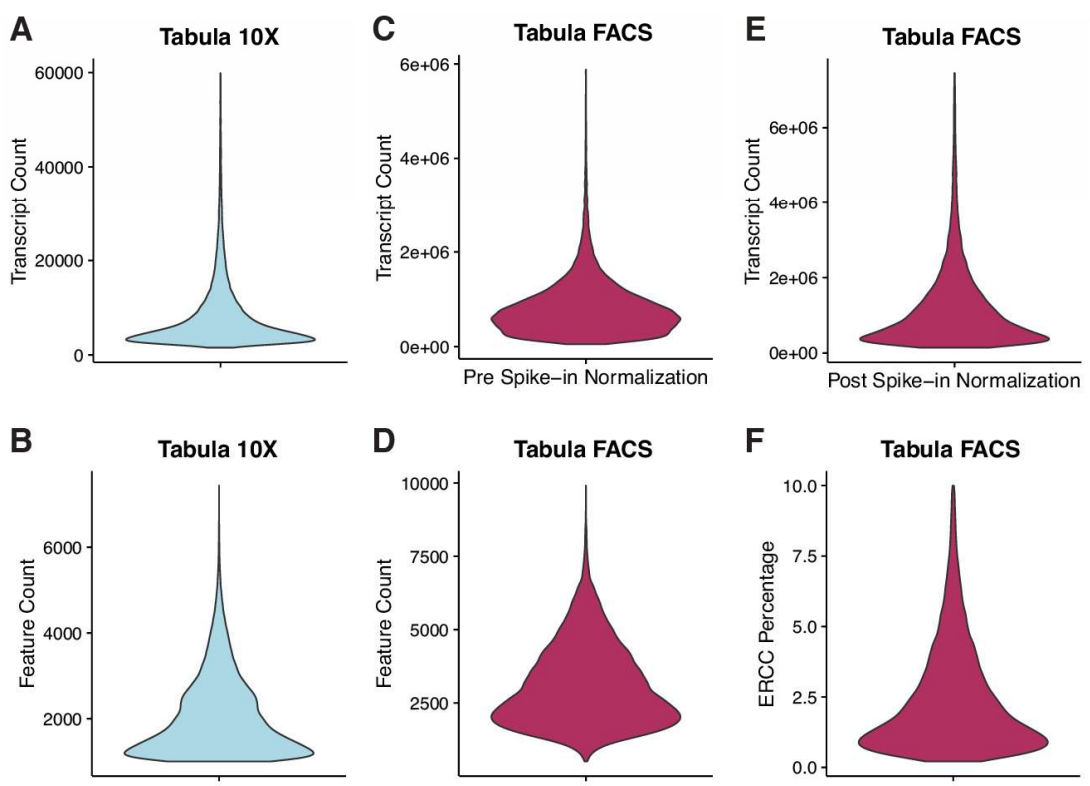

H
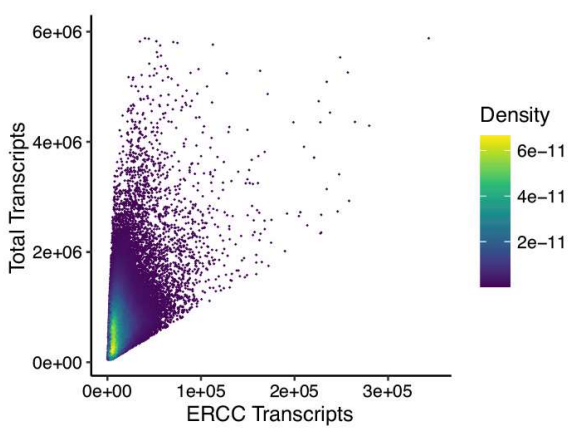

I

J
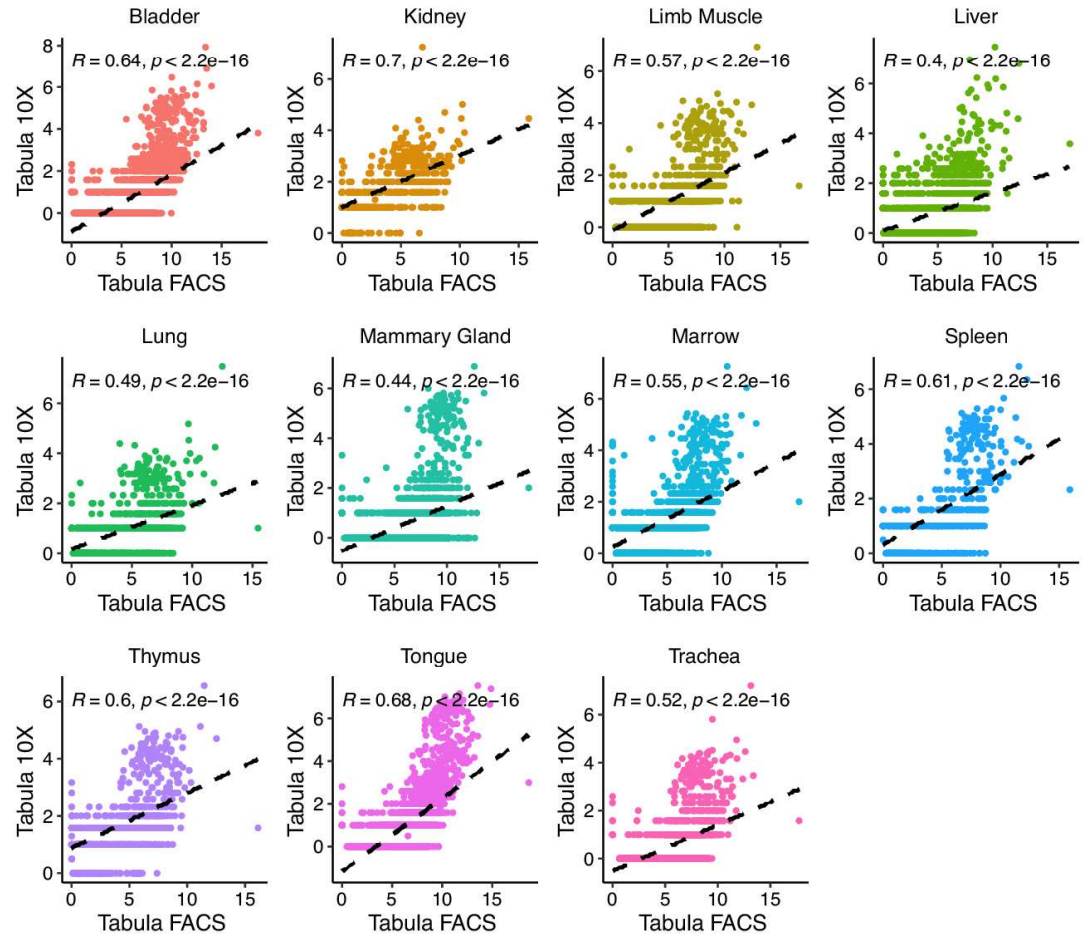

G

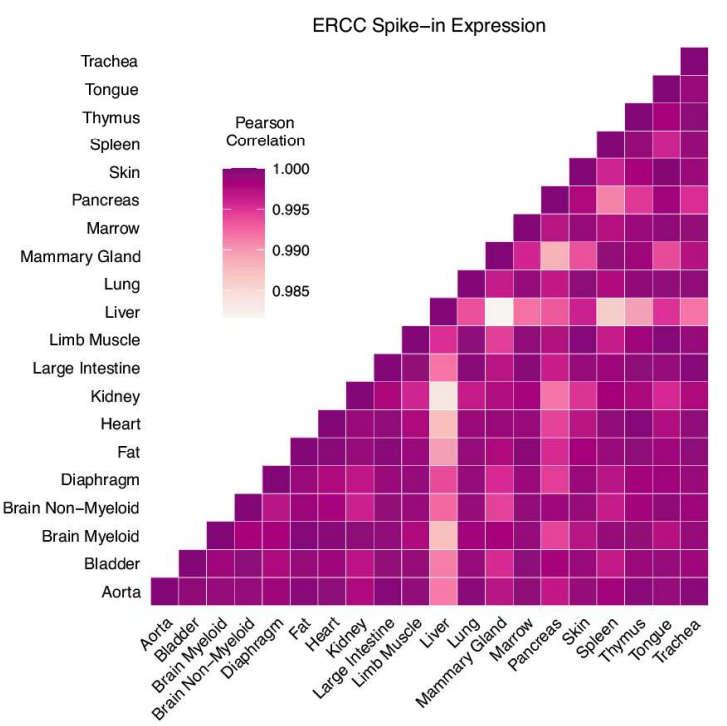

K

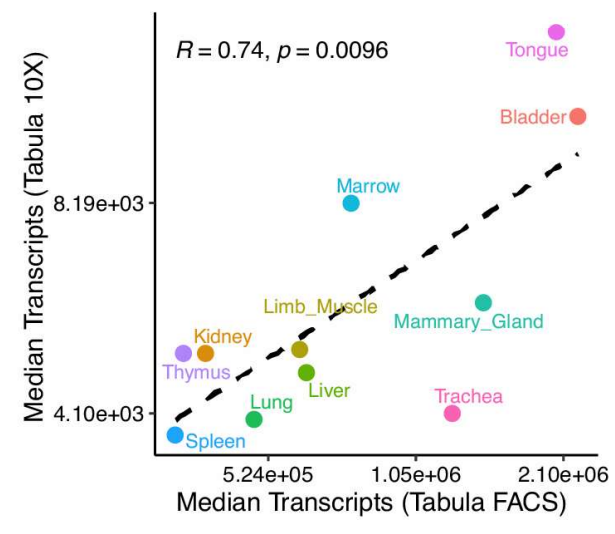

\section{Supplementary Figure 6}


bioRxiv preprint doi: https://doi.org/10.1101/2021.12.13.472426; this version posted December 13,2021 . The copyright holder for this preprint (which was not certified by peer review) is the author/funder. All rights reserved. No reuse allowed without permission.

A
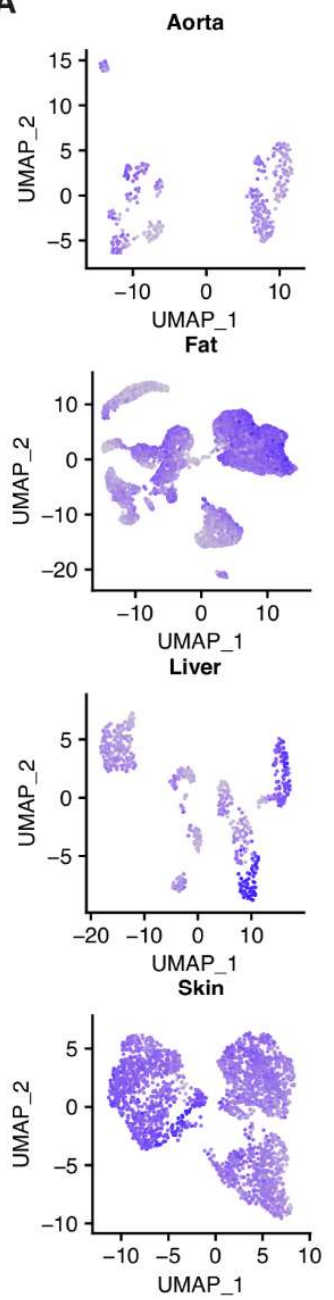

B
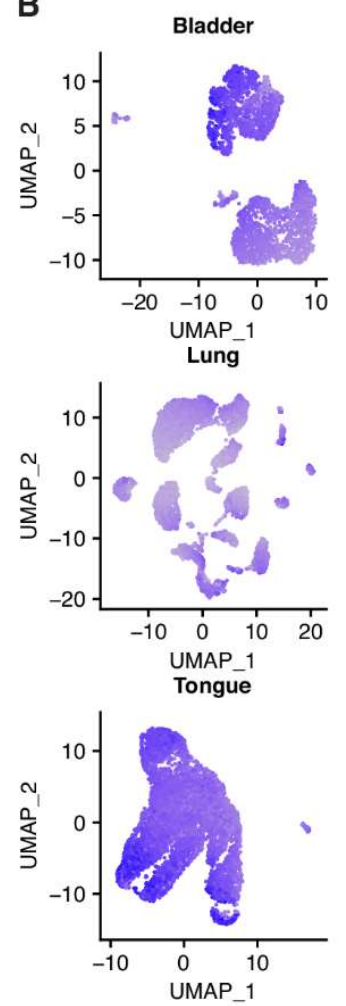
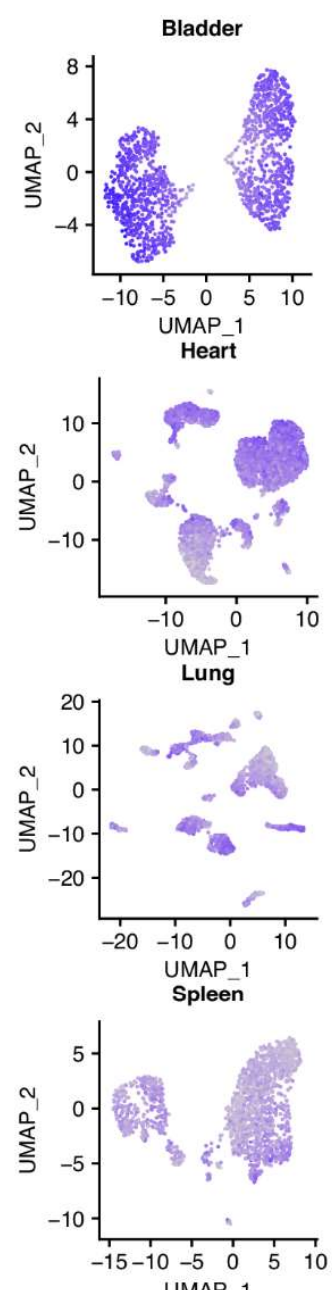

UMAP_1
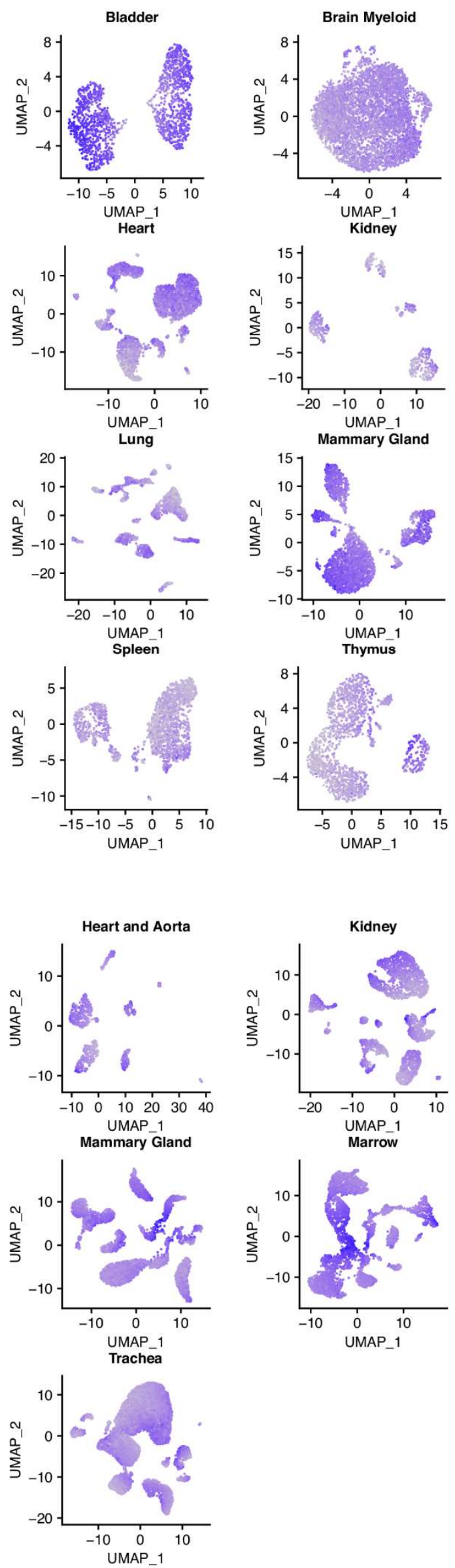
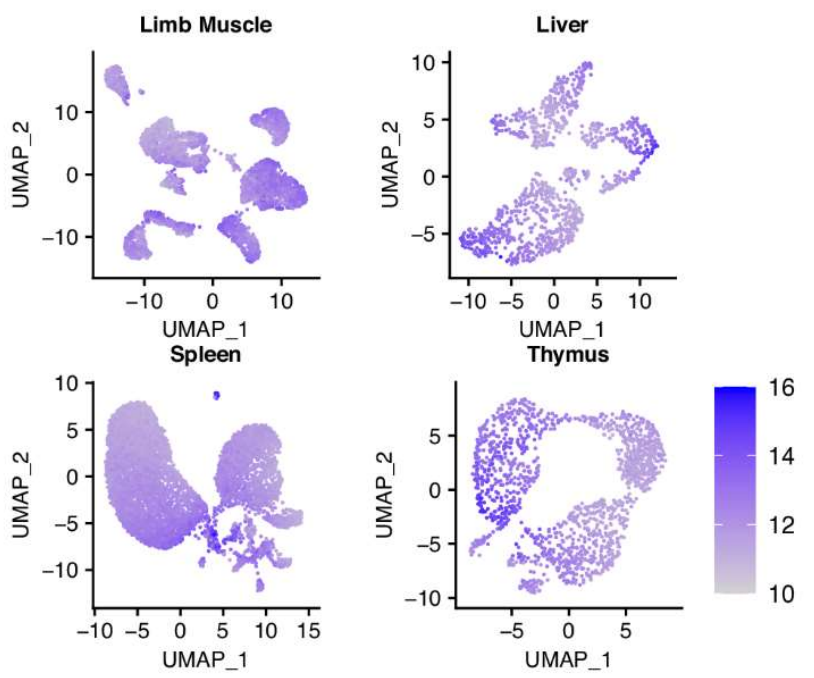
bioRxiv preprint doi: https://doi.org/10.1101/2021.12.13.472426; this version posted December 13, 2021. The copyright holder for this preprint (which was not certified by peer review) is the author/funder. All rights reserved. No reuse allowed without permission.

A
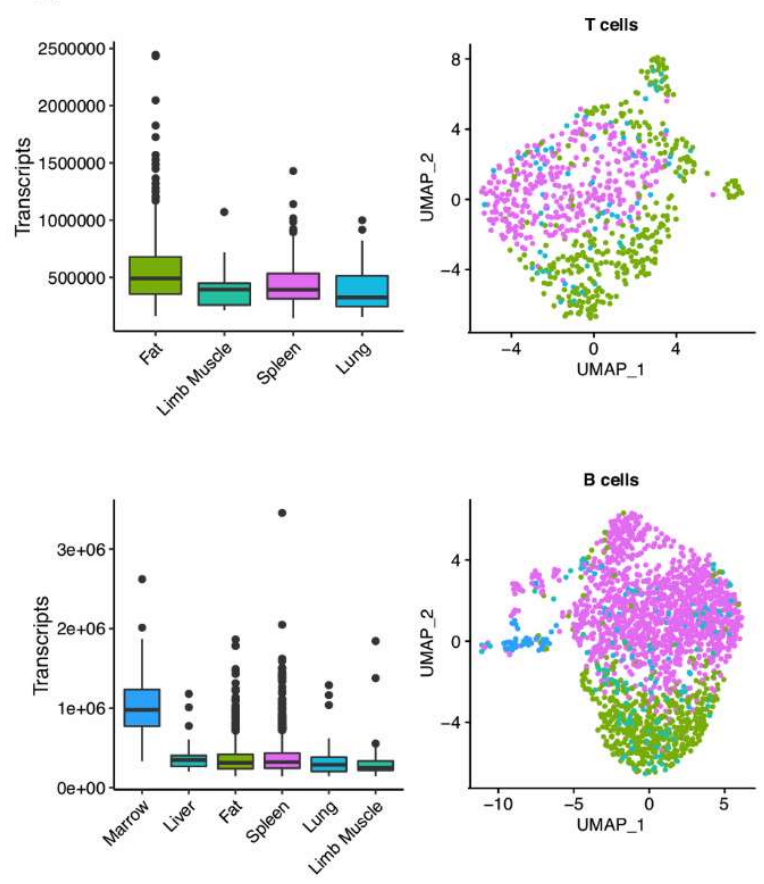

- Fat

- Limb_Muscle

- Lung

Spleen

- Liver

Liver
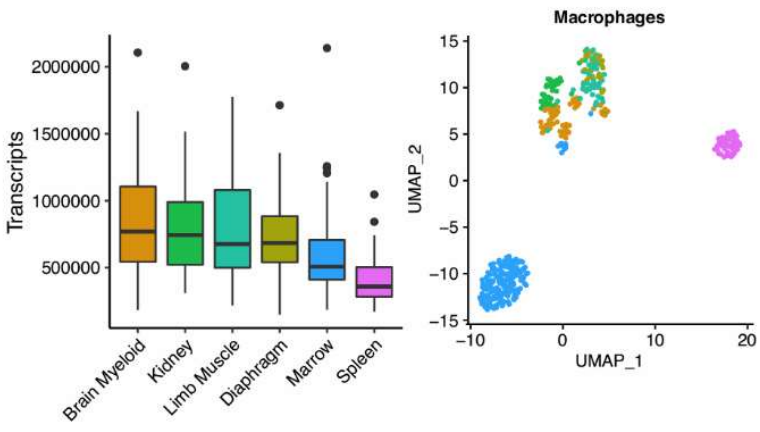

- Brain_Myeloid - Limb_Muscle - Diaphragm

- Spleen

- Kidney
B
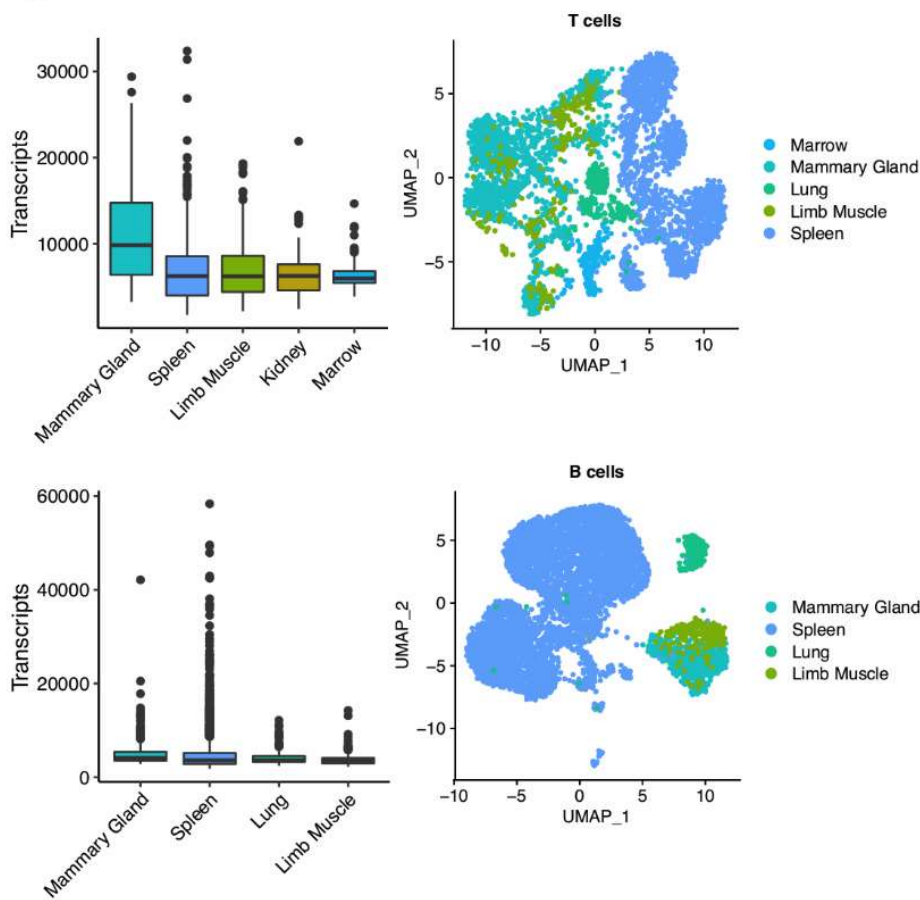

Mammary Gland - Spleen - Limb Muscle
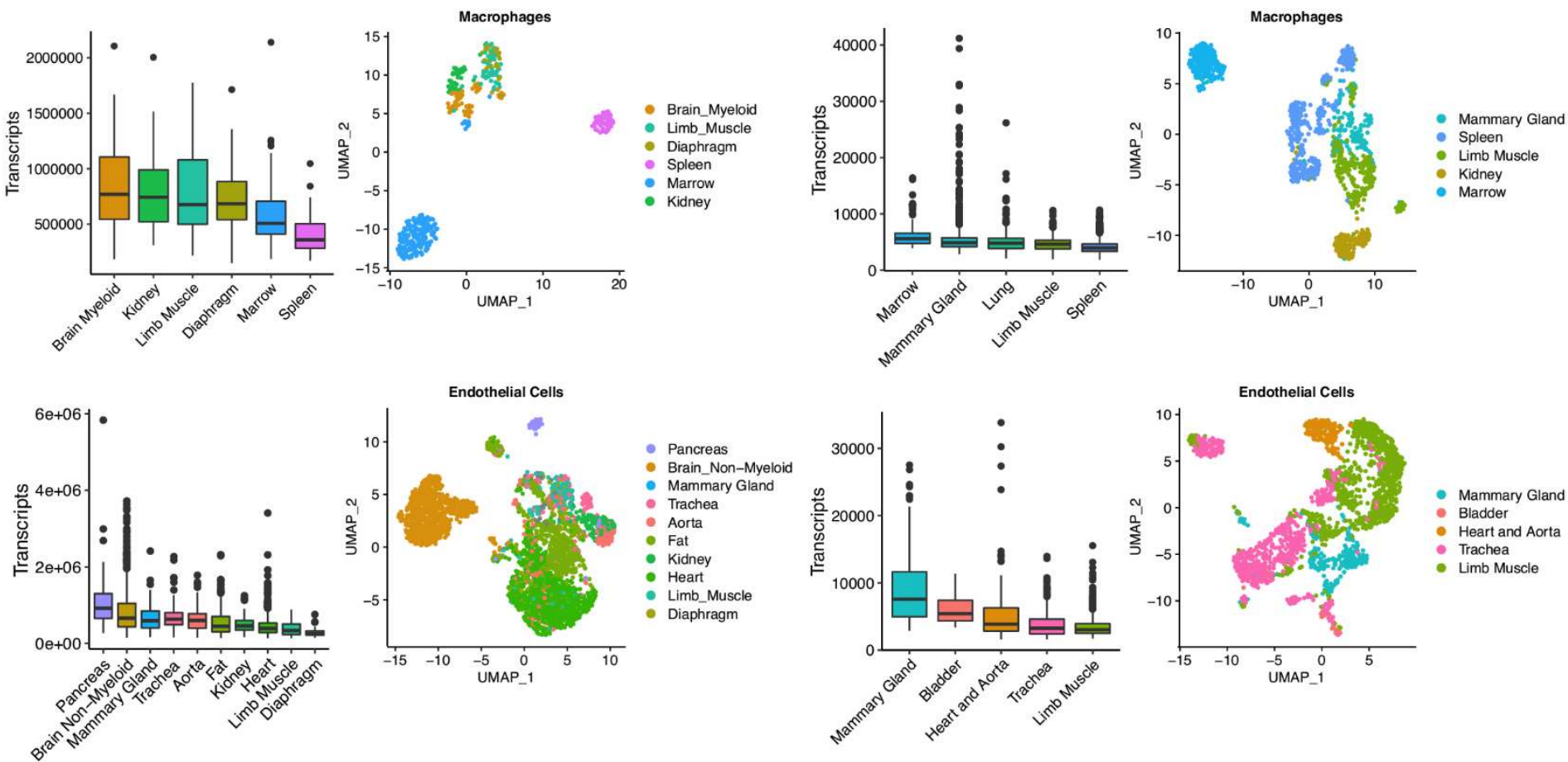
bioRxiv preprint doi: https://doi.org/10.1101/2021.12.13.472426; this version posted December 13, 2021. The copyright holder for this preprint (which was not certified by peer review) is the author/funder. All rights reserved. No reuse allowed without permission.

A

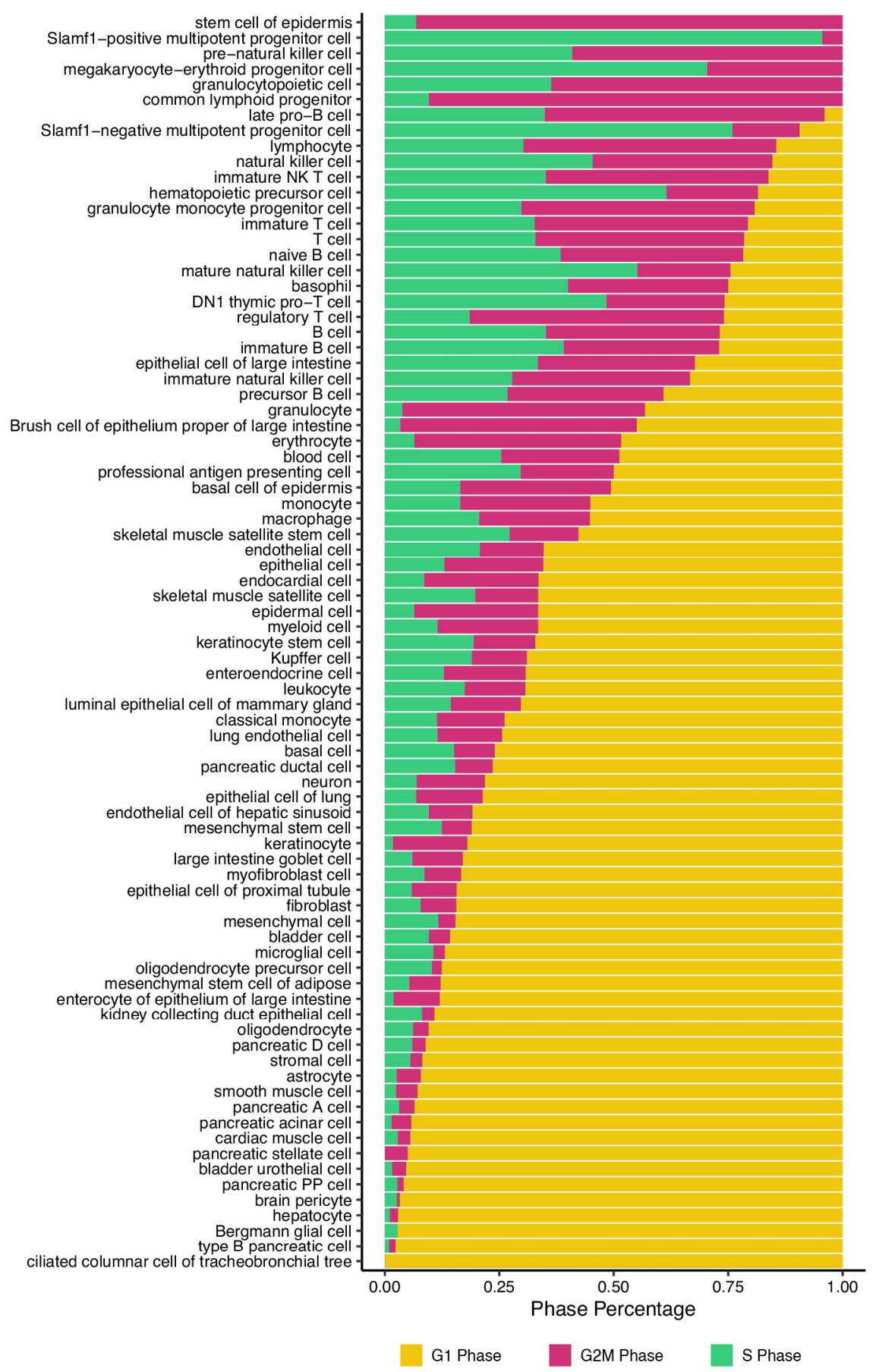

stem cell of epidermis pretent progenitor cell rythroid progenitor cell granulocytopoietic cell common lymphoid progenito lymphocyte pro-T cell helial cell of large intestine immature natural killer cell recursor B cell granulocyte erythrocyte professional antigen presenting cell satellite cell upffer cell of mammar pancreatic ducal cell keratinocyte proximal tubule (adder ce mesenchymal stem cell of adipose kidney epithelium of large intest ce stromal cell astrocyte mooth muscle ce pancreatic A ce creatic stellate cell eatic PP cellbrain pericyte B pancreatic cell

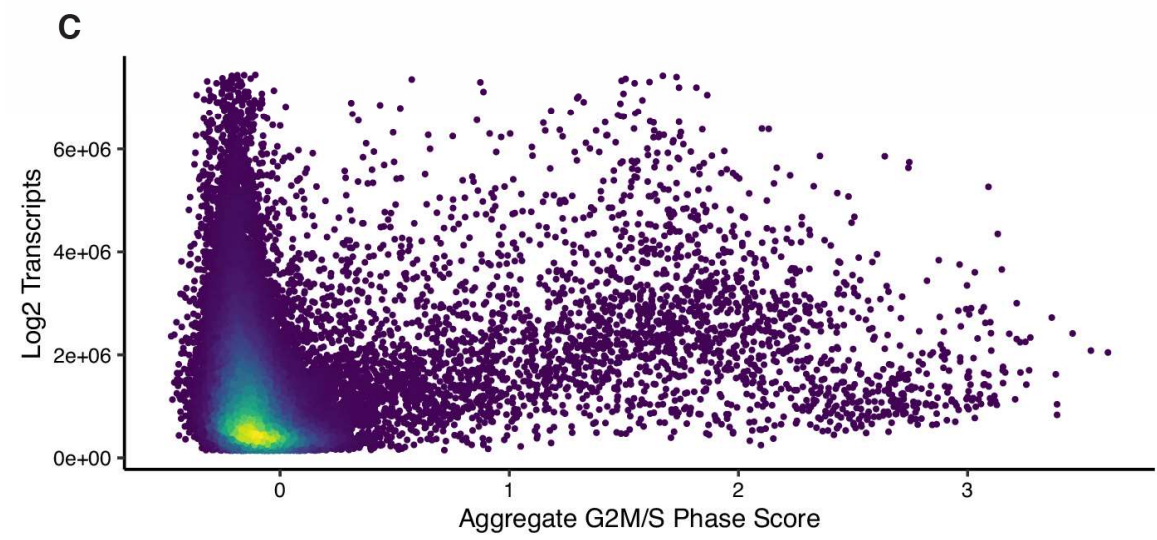

Density

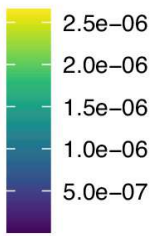


bioRxiv preprint doi: https://doi.org/10.1101/2021.12.13.472426; this version posted December 13, 2021. The copyright holder for this preprint (which was not certified by peer review) is the author/funder. All rights reserved. No reuse allowed without permission.

A

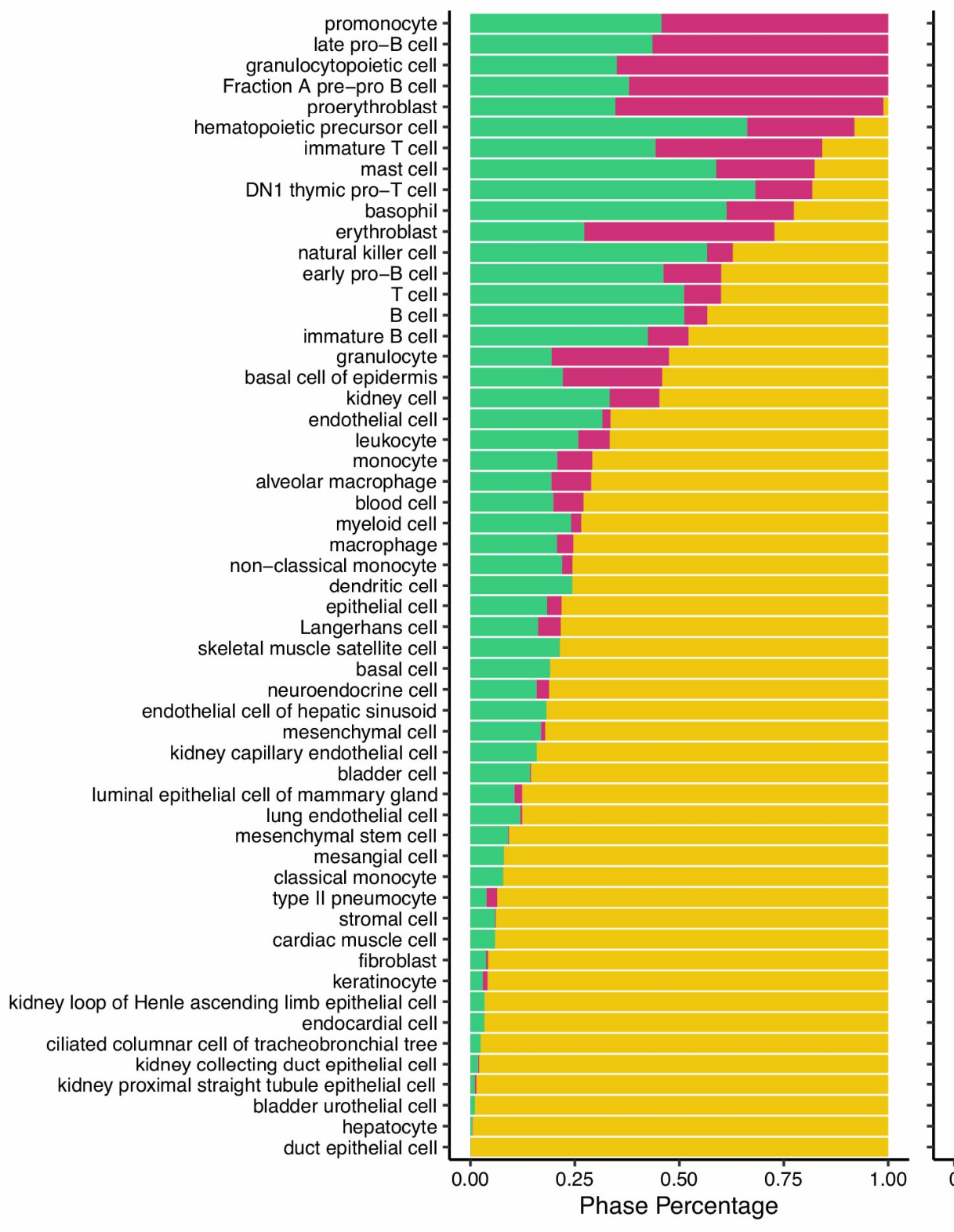

B

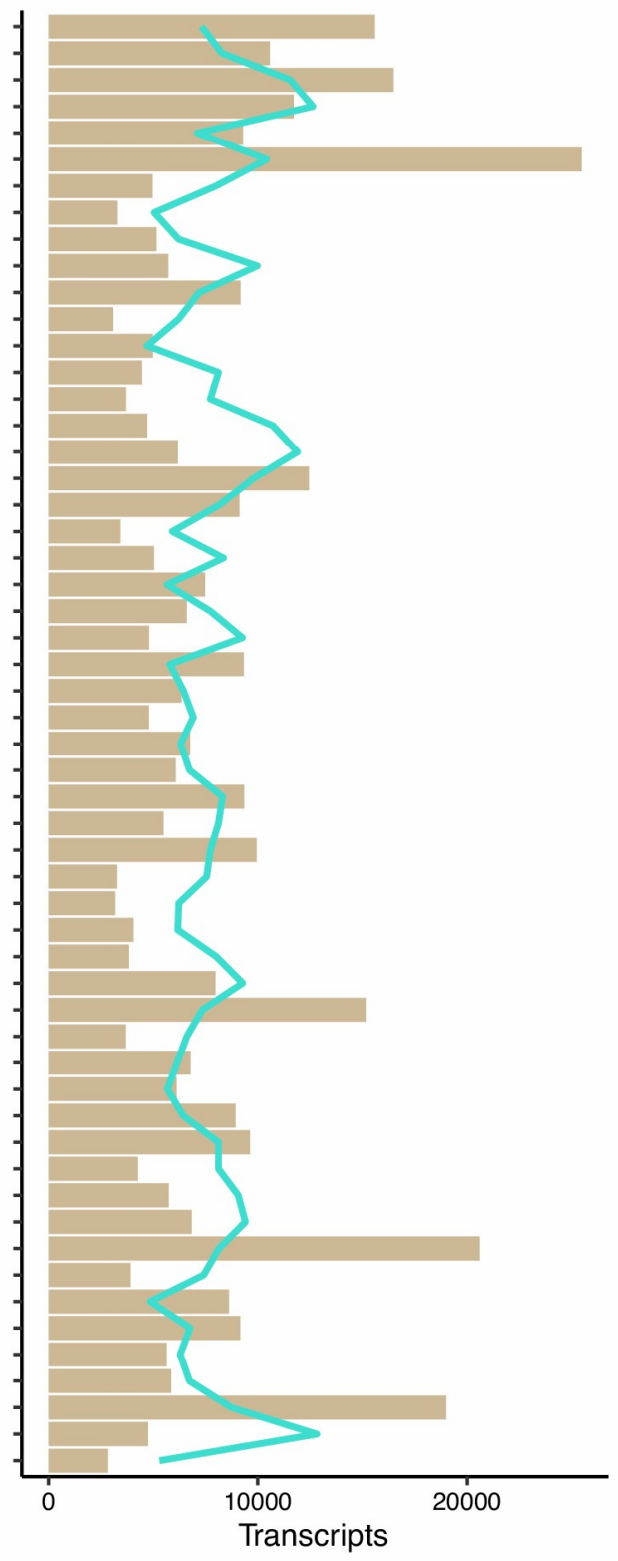

G1 Phase G2M Phase $\square$ S Phase

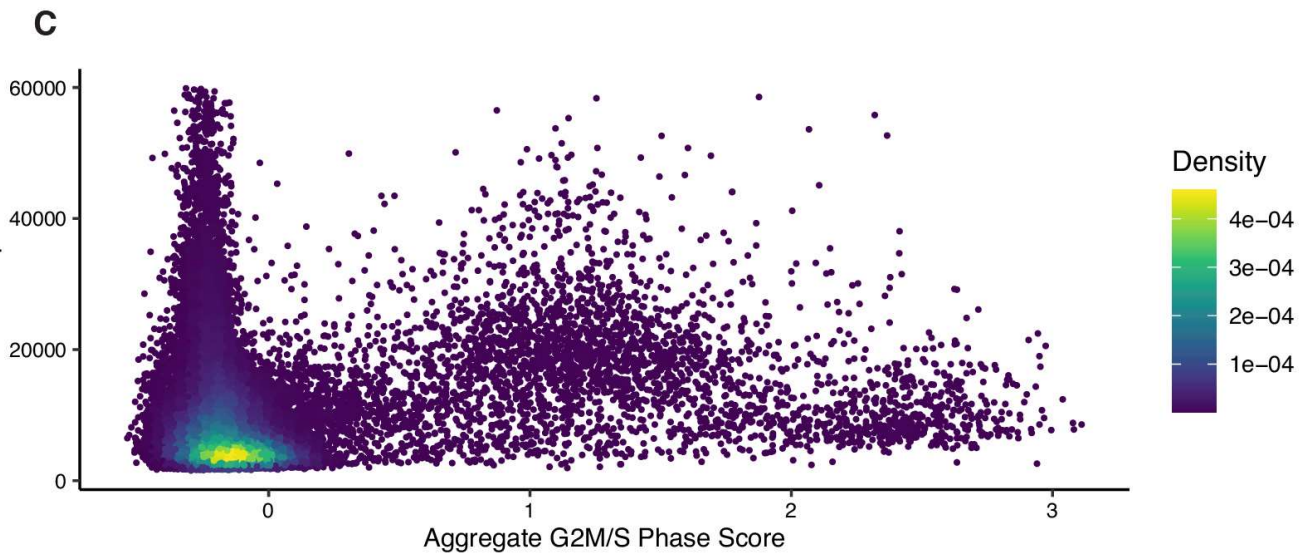


bioRxiv preprint doi: https://doi.org/10.1101/2021.12.13.472426; this version posted December 13, 2021. The copyright holder for this preprint (which was not certified by peer review) is the author/funder. All rights reserved. No reuse allowed without permission.

A

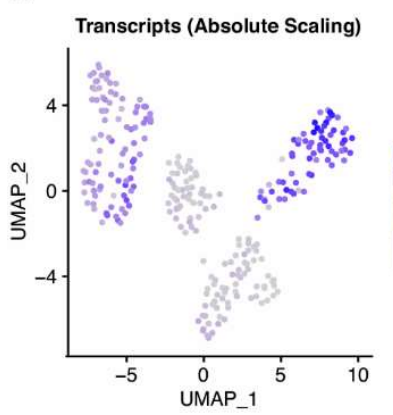

E

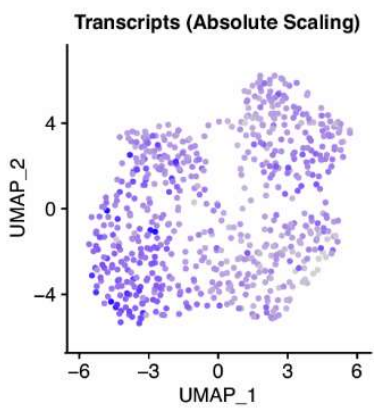

H

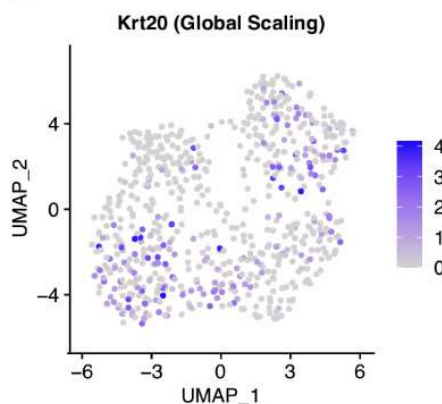

I

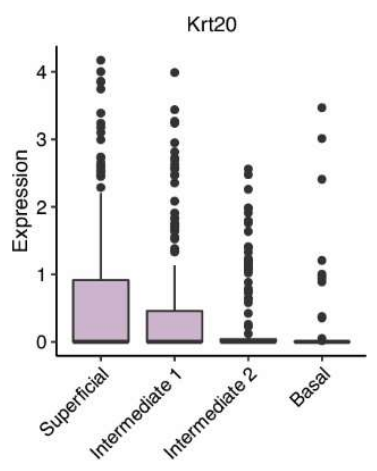

B

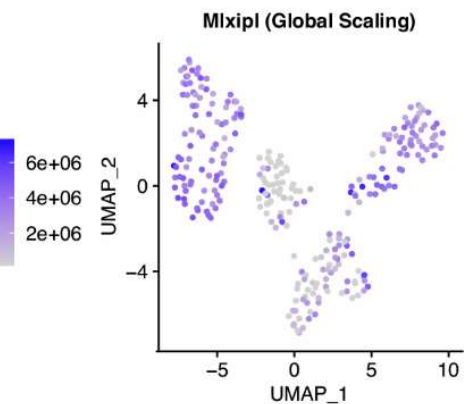

$\mathbf{F}$

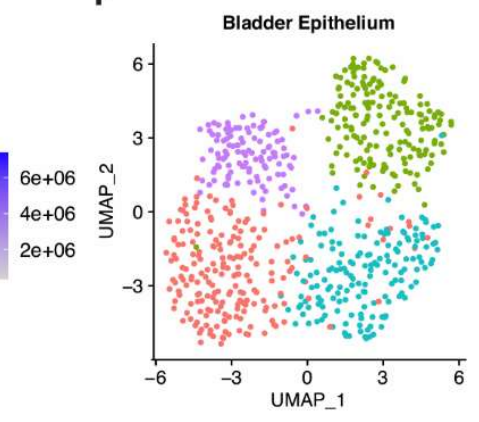

$\mathbf{J}$

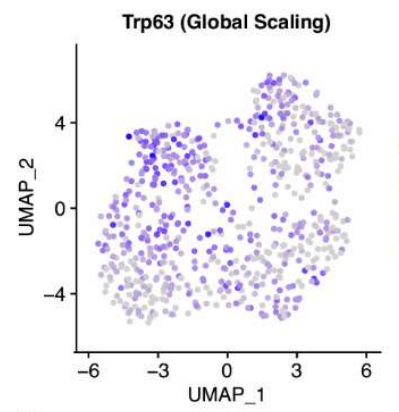

K

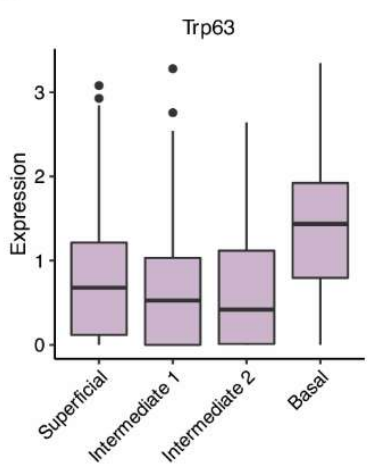

C
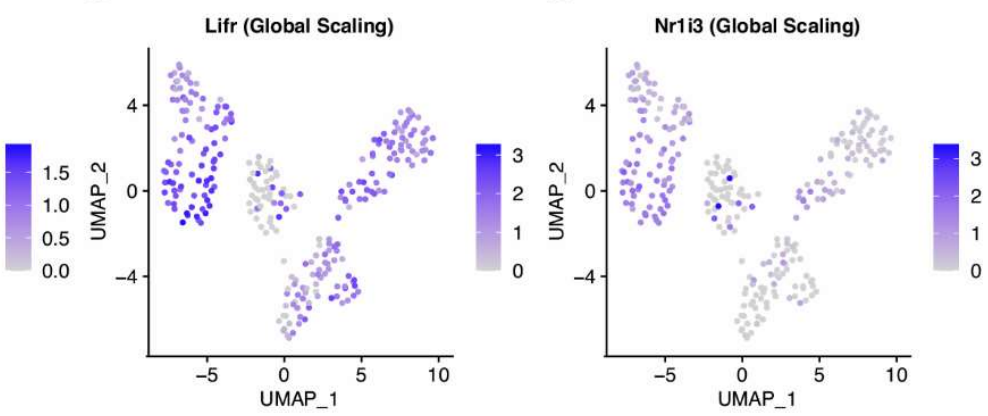

G

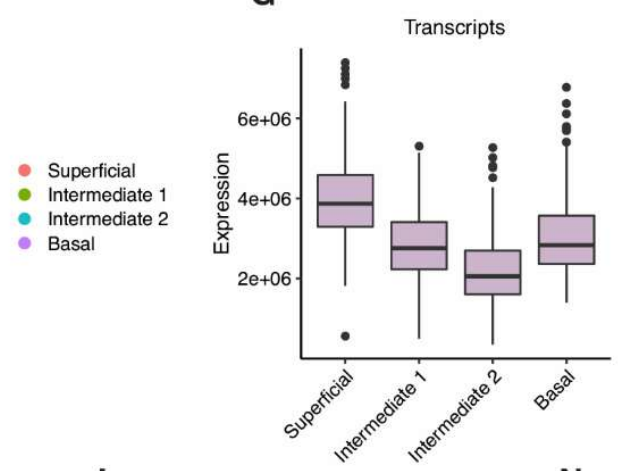

L

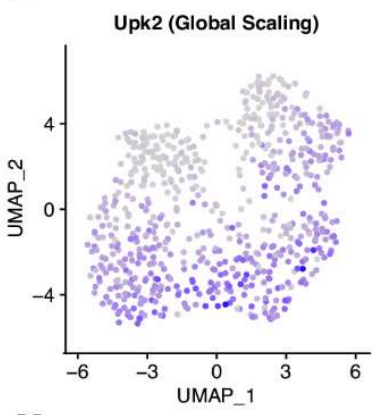

M
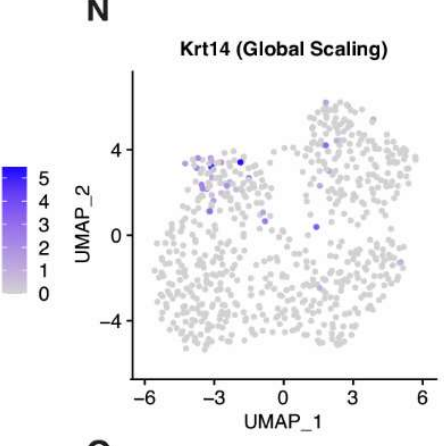

O
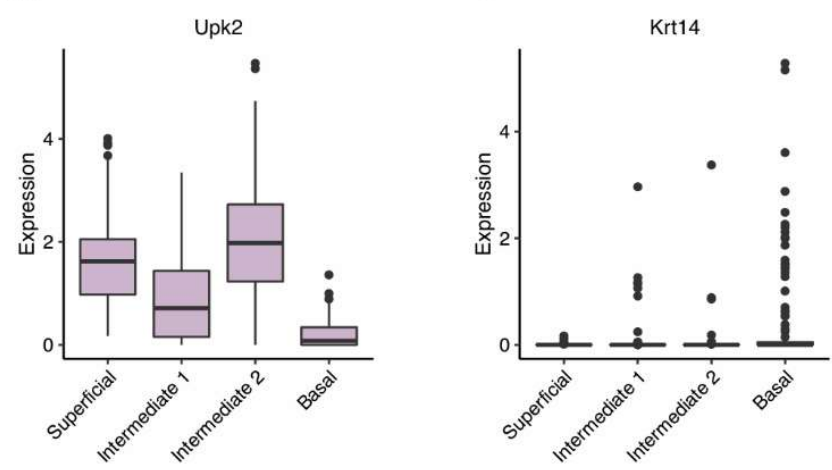
bioRxiv preprint doi: https://doi.org/10.1101/2021.12.13.472426; this version posted December 13, 2021. The copyright holder for this preprint (which was not certified by peer review) is the author/funder. All rights reserved. No reuse allowed without permission.

A

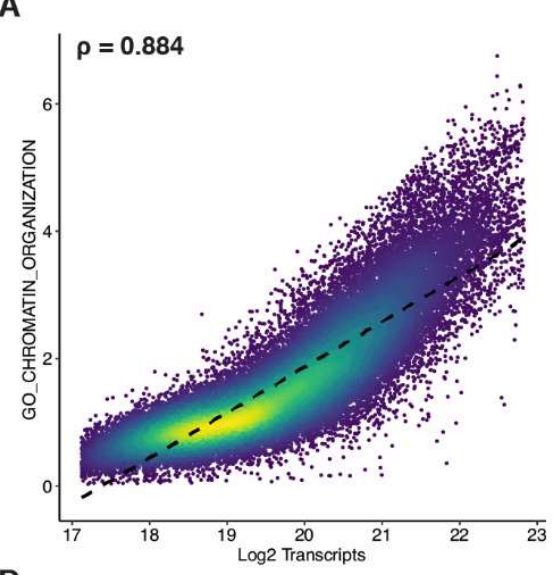

D

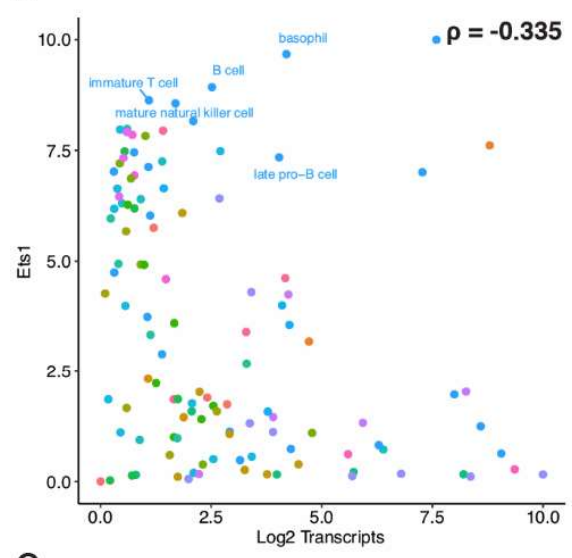

G

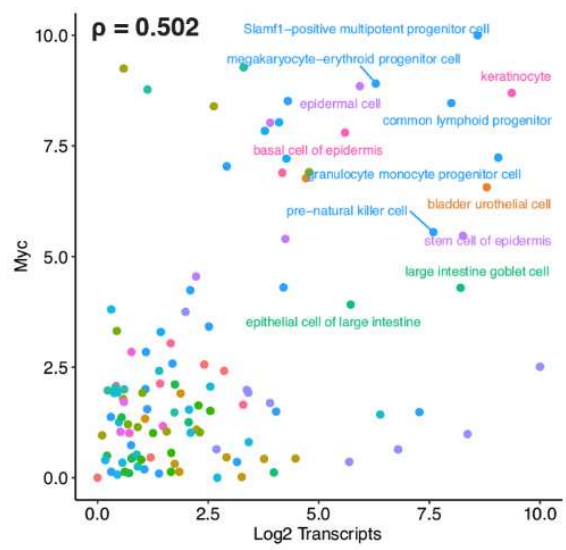

$\mathbf{J}$

MYC 19030024 ChIP-ChIP MESCs Mouse EKLF 21900194 ChIP-Seq ERYTHROCYTE Mouse MYC 18358816 ChIP-ChIP MESCs MouseE2F1 18555785 ChIP-Seq MESCs Mouse. ZFX 18555785 ChIP-Seq MESCs Mouse CREM 20920259 ChIP-Seq GC1-SPG Mouse MYC 18555785 ChIP-Seq MESCs MouseXRN2 22483619 ChIP-Seq HELA Human. XRN2 22483619 ChIP-Seq HELA Human-
MYC 19079543 ChIP-ChIP MESCs MouseMYC 19079543 ChIP-ChIP MESCs Mouse NELFA 20434984 ChIP-Seq ESCs Mouse TAL1 20887958 ChIP-Seq HPC-7 Mouse TTF2 22483619 ChIP-Seq HELA Human ETS1 20019798 ChIP-Seq JURKAT Human JARID1A 20064375 ChIP-Seq MESCs Mouse CREB1 23762244 ChIP-Seq HIPPOCAMPUS Rat VDR 23849224 ChIP-Seq CD4+ Human
FLI1 21571218 ChIP-Seq MEGAKARYOCYTES Human
YY1 23942234 ChIP-Seq MYOBLASTS AND MYOTUB... YY1 23942234 ChIP-Seq MYOBLASTS AND MYOTUB... KDM5B 21448134 ChIP-Seq MESCs Mouse CREB1 20920259 ChIP-Seq GC1-SPG Mouse
B

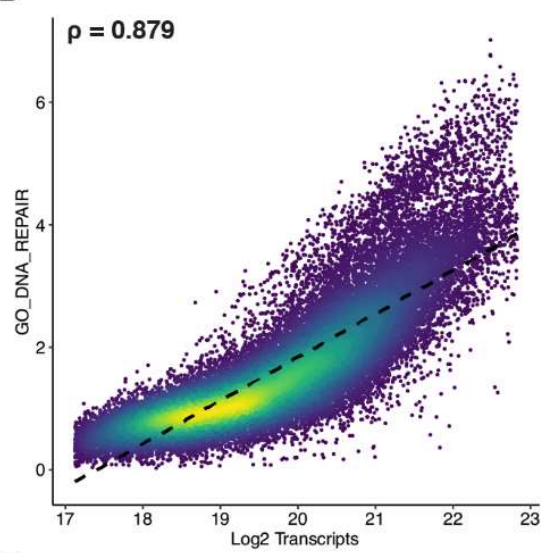

E

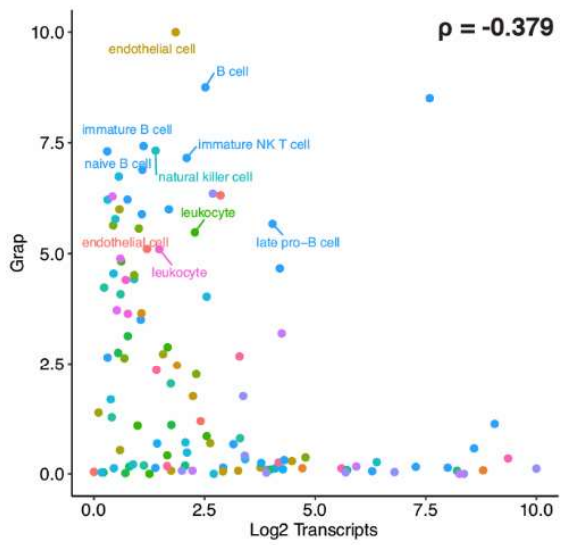

H

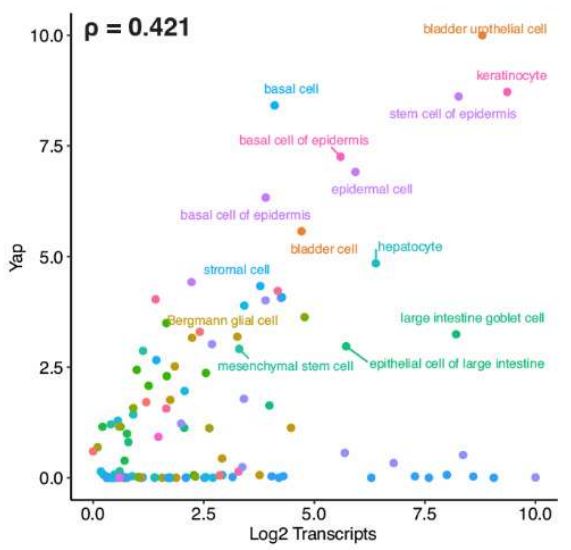

ChEA Transcription Factors
C

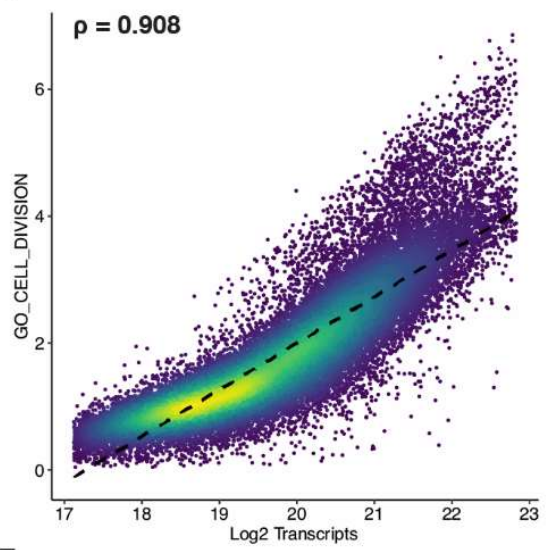

F

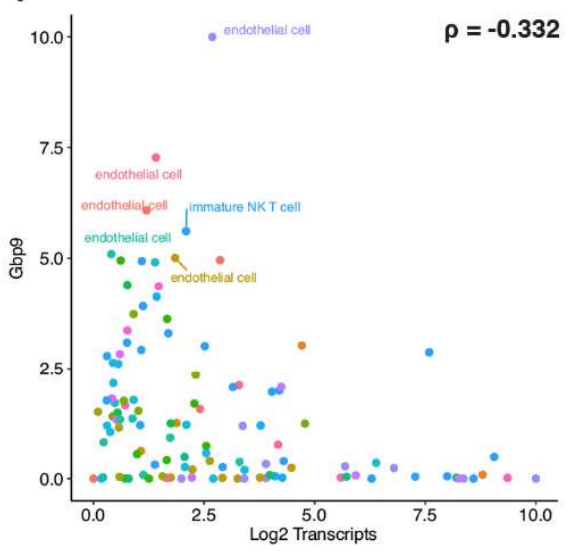

I

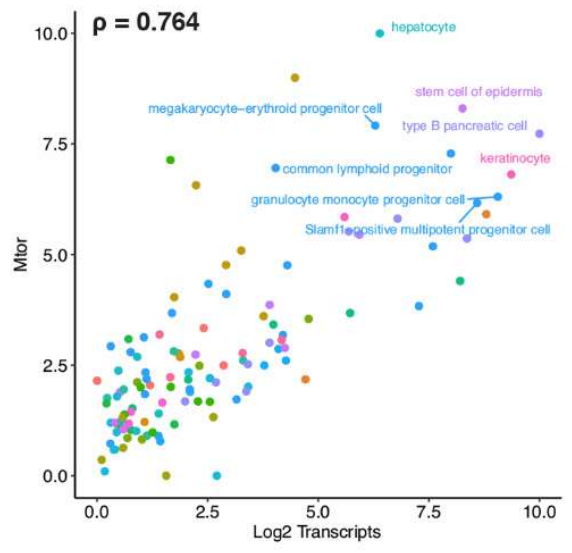

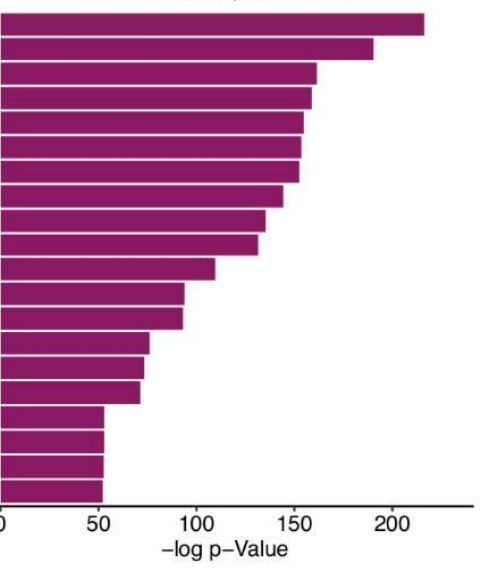


bioRxiv preprint doi: https://doi.org/10.1101/2021.12.13.472426; this version posted December 13, 2021. The copyright holder for this preprint (which was not certified by peer review) is the author/funder. All rights reserved. No reuse allowed without permission.

A
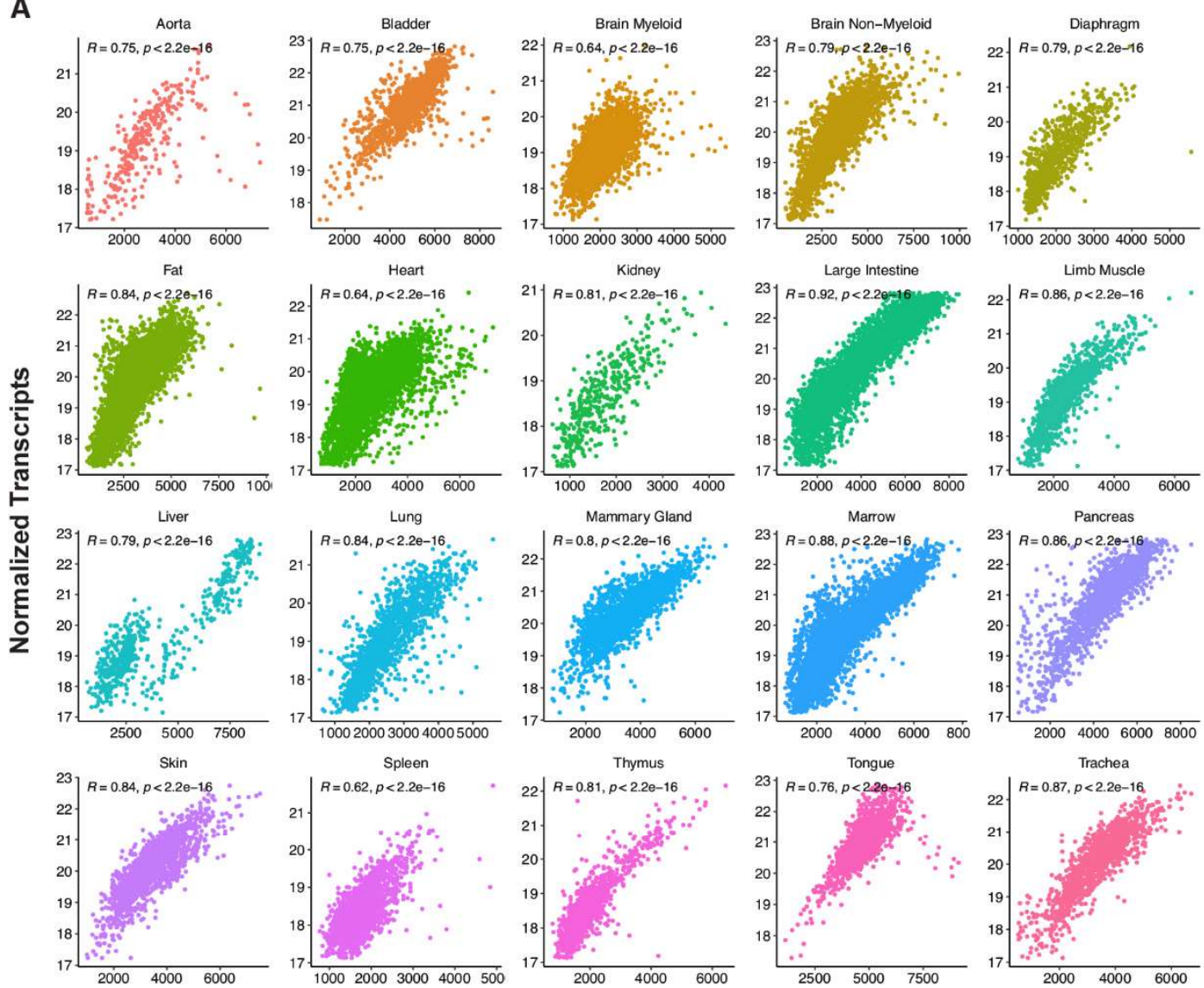

Normalized Feature Counts

B

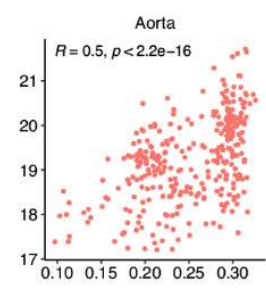

Fat

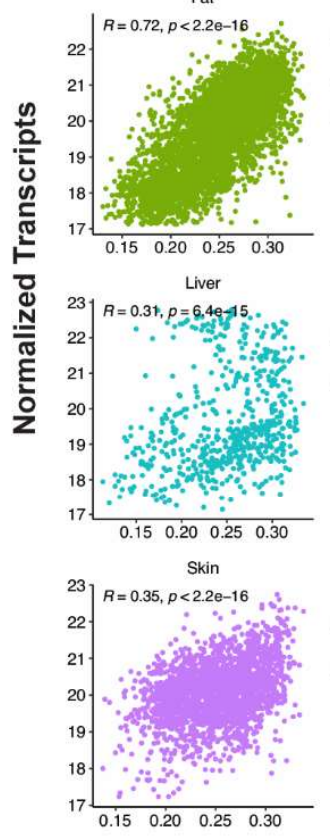

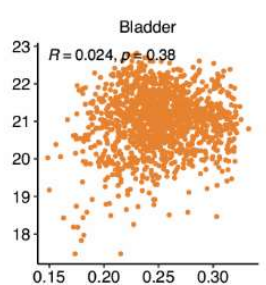

Heart

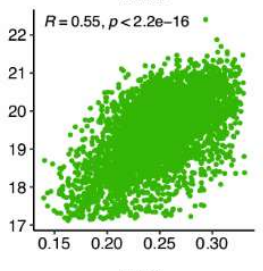

Lung

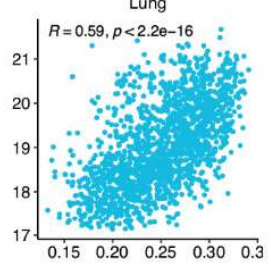

Spleen

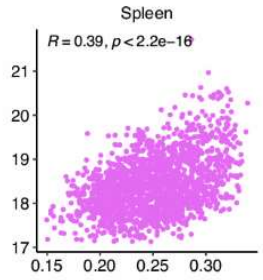

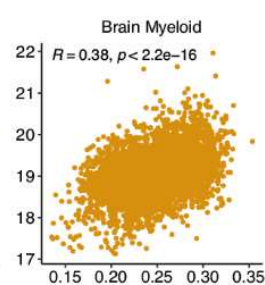

Kidney
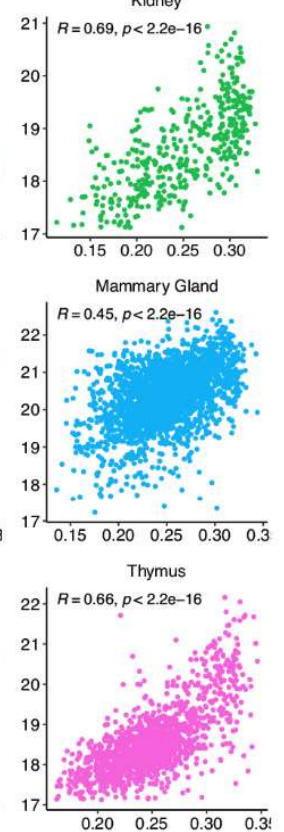
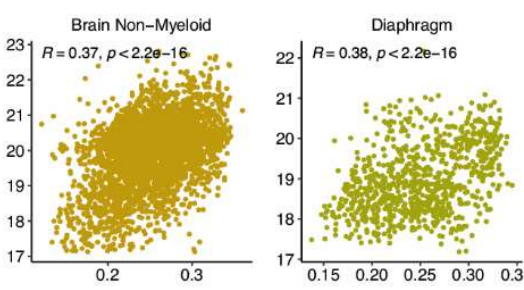

Large Intestine

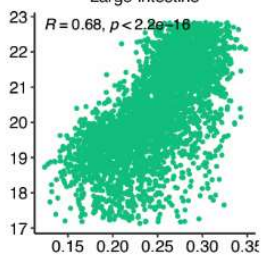

Limb Muscle
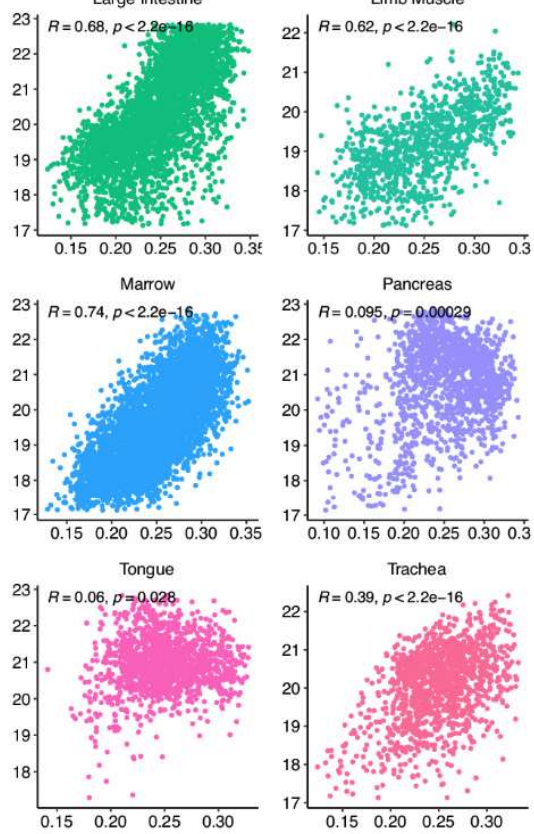

Trachea

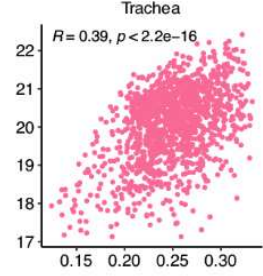


bioRxiv preprint doi: https://doi.org/10.1101/2021.12.13.472426; this version posted December 13, 2021. The copyright holder for this preprint (which was not certified by peer review) is the author/funder. All rights reserved. No reuse allowed without permission.

A

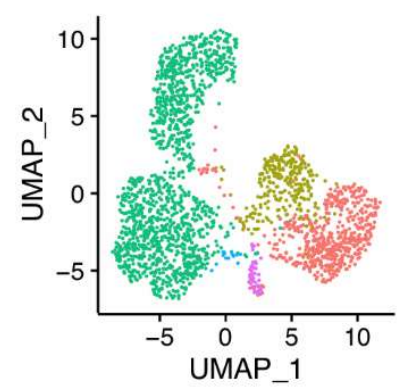

D

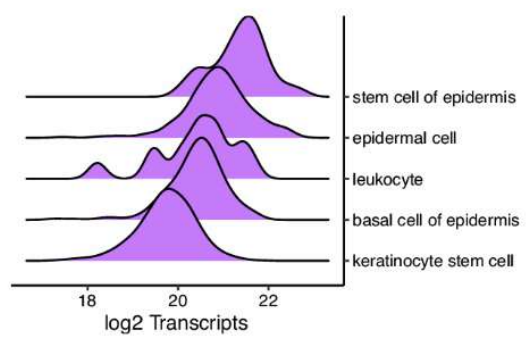

B

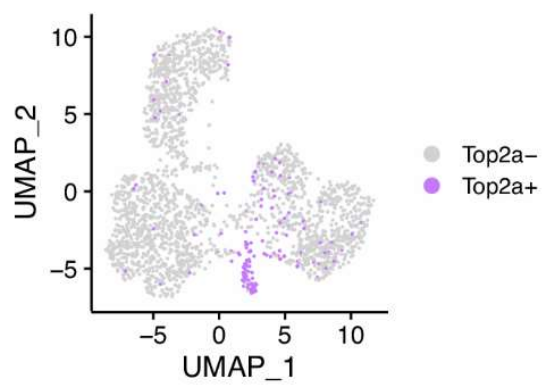

C

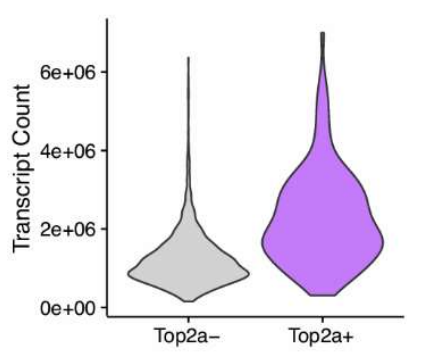

E
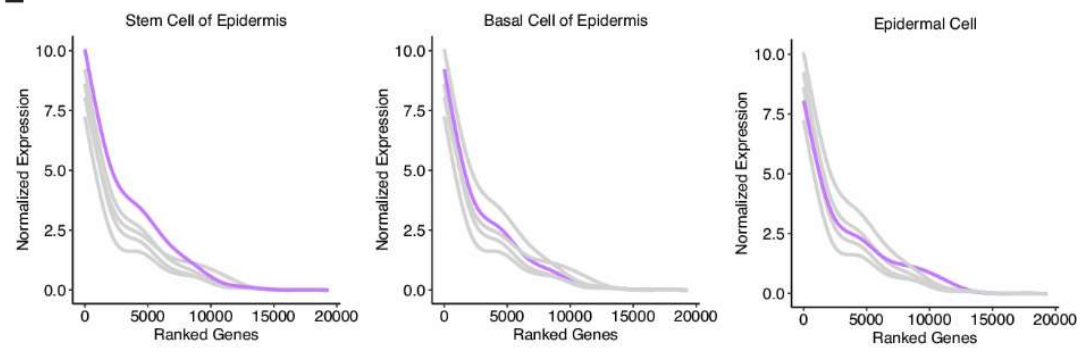

$\mathbf{F}$

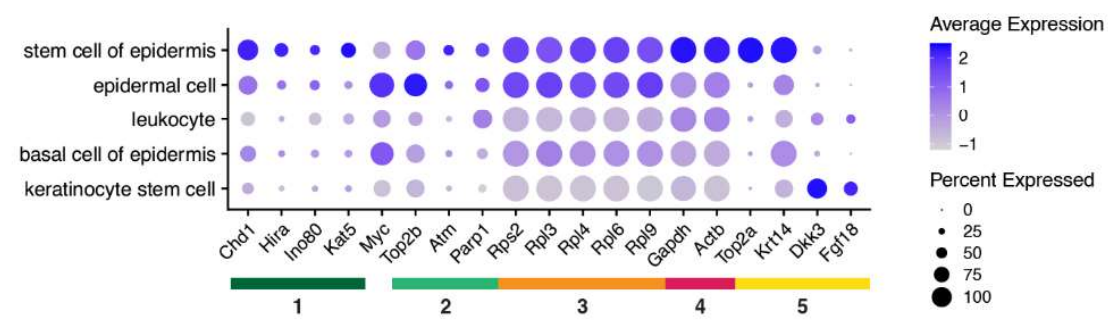

G
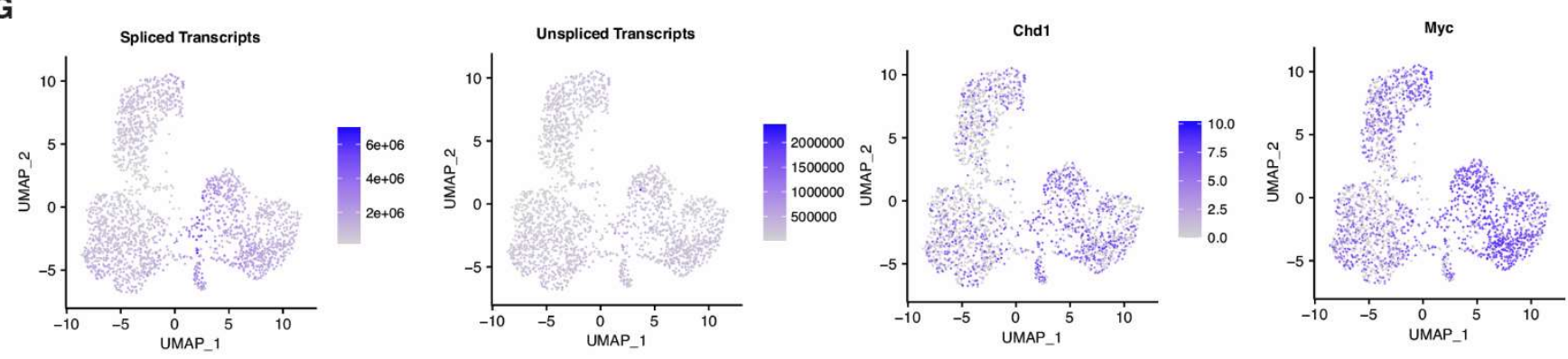

H
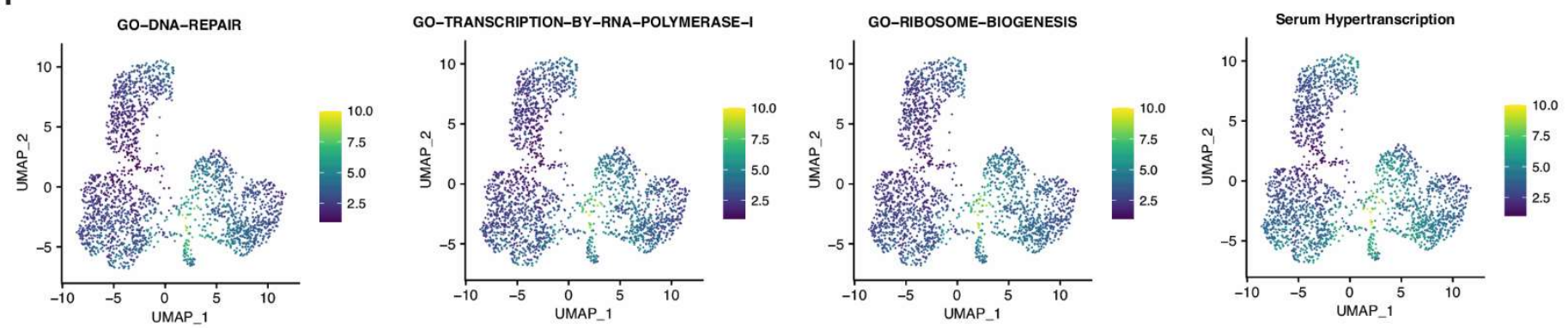
bioRxiv preprint doi: https://doi.org/10.1101/2021.12.13.472426; this version posted December 13, 2021. The copyright holder for this preprint (which was not certified by peer review) is the author/funder. All rights reserved. No reuse allowed without permission.

A

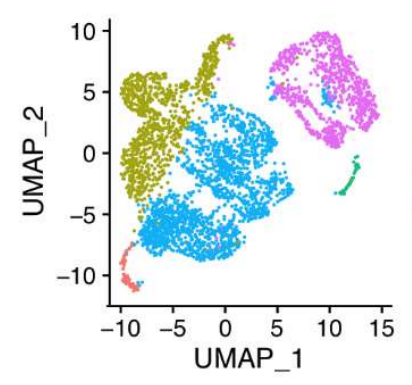

D

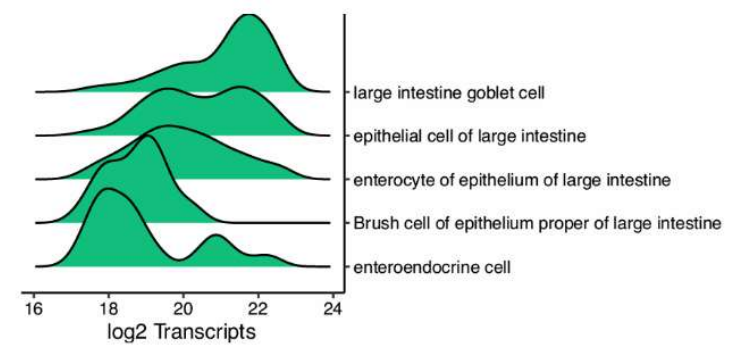

enteroendocrine cell

epithelial cell of large intestine
B

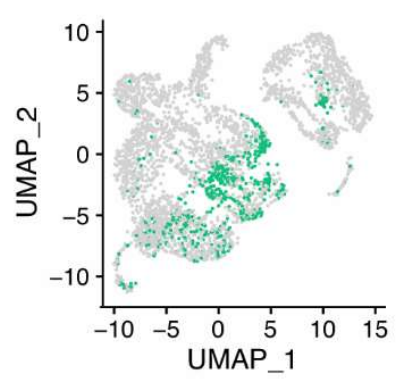

E

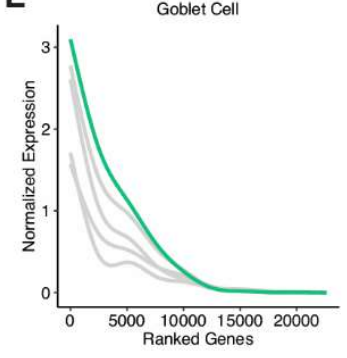

C

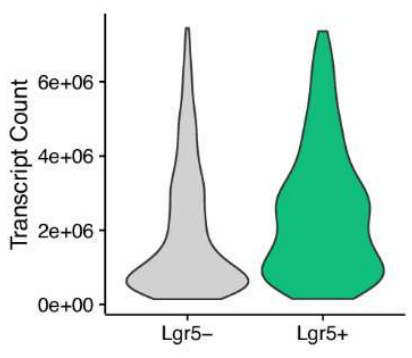

F

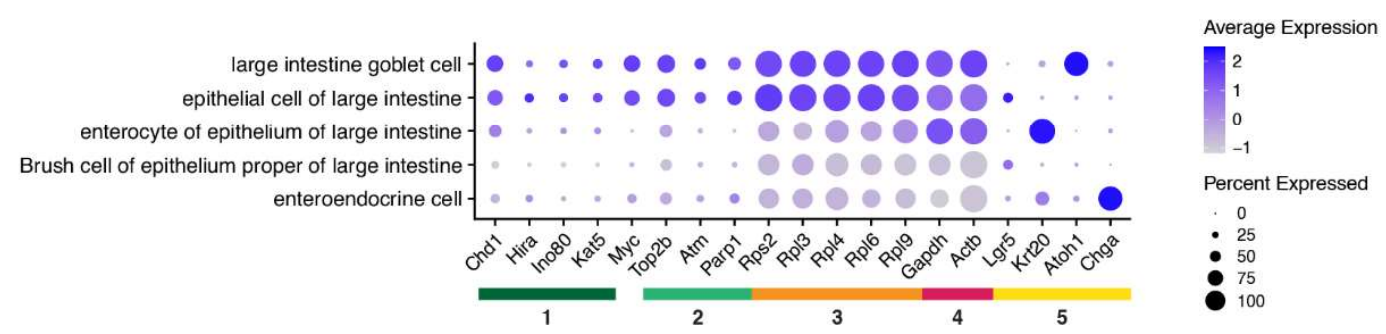

G
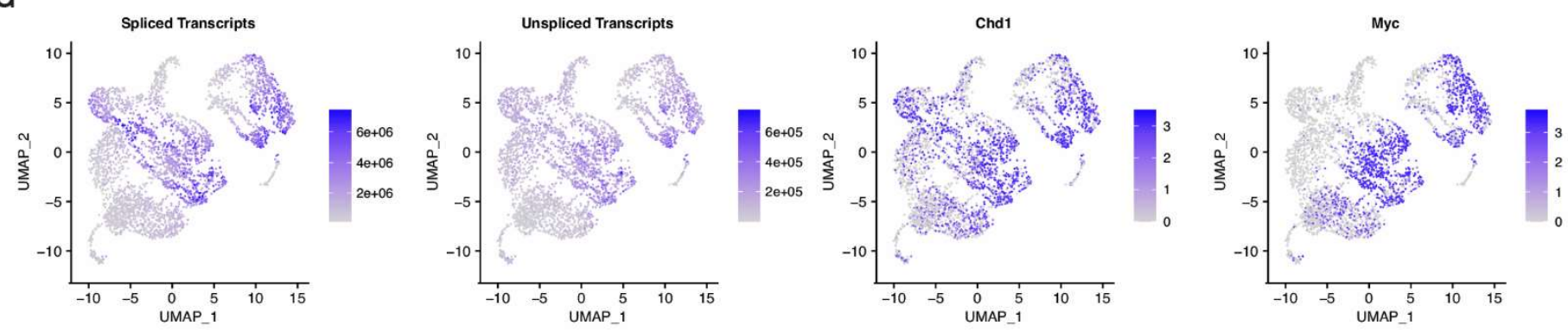

H
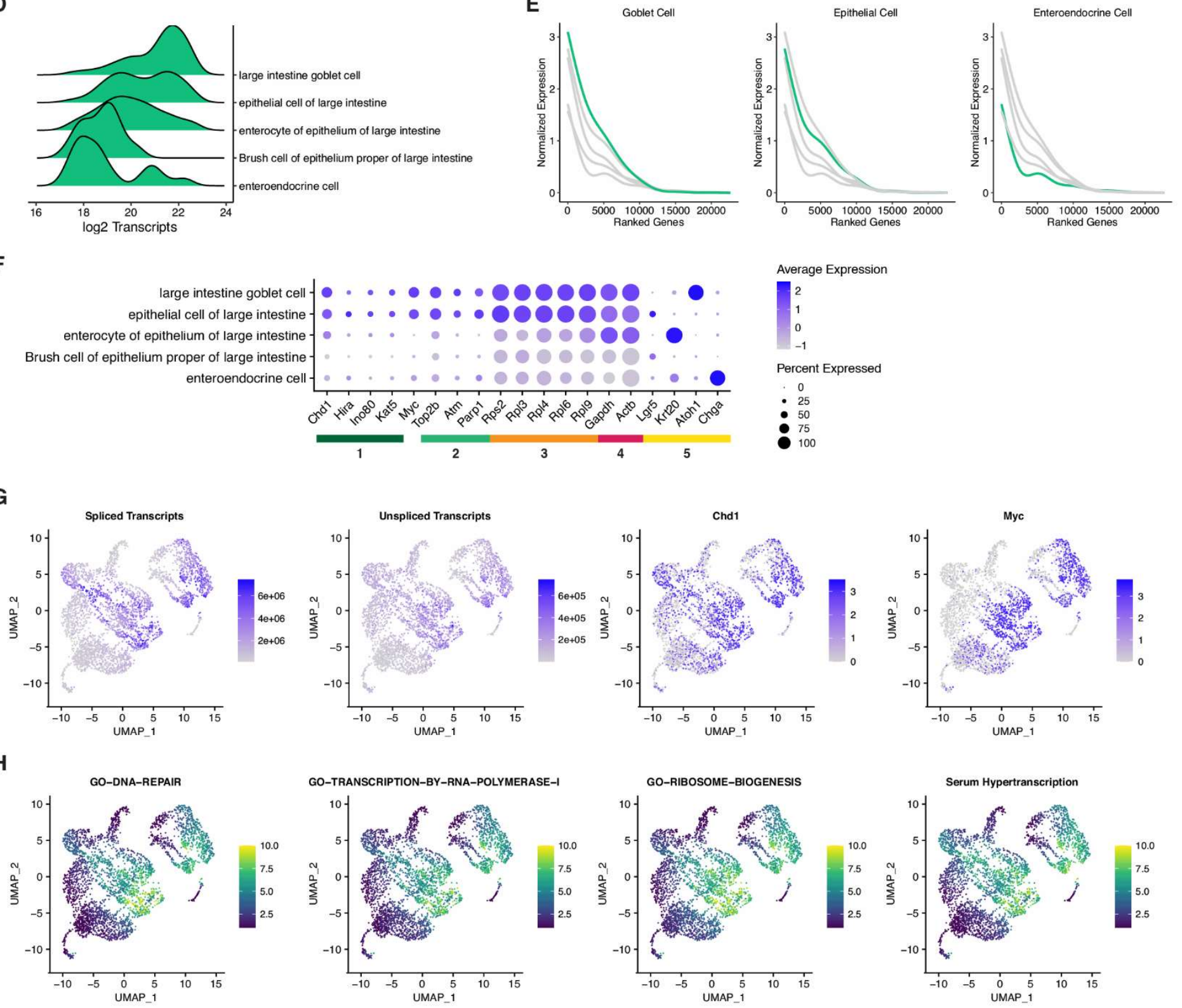

Brush cell of epithelium proper of large intestine

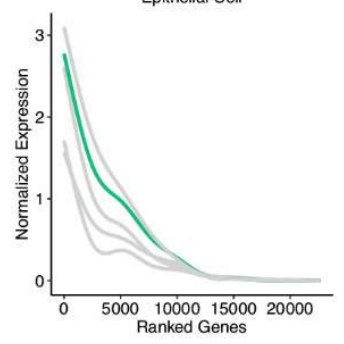


bioRxiv preprint doi: https://doi.org/10.1101/2021.12.13.472426; this version posted December 13, 2021. The copyright holder for this preprint (which was not certified by peer review) is the author/funder. All rights reserved. No reuse allowed without permission.

A

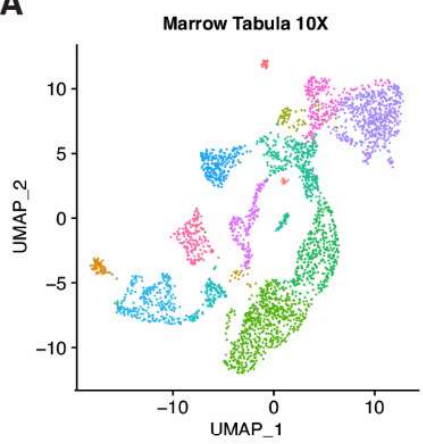

C

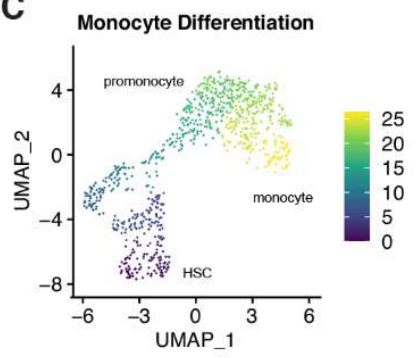

D

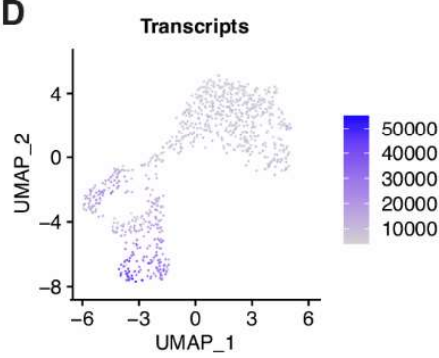

I

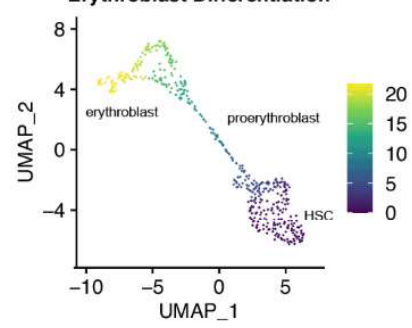

J

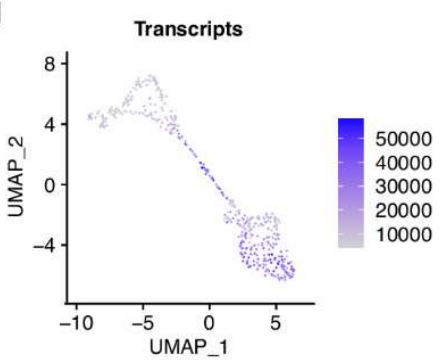

\section{E}
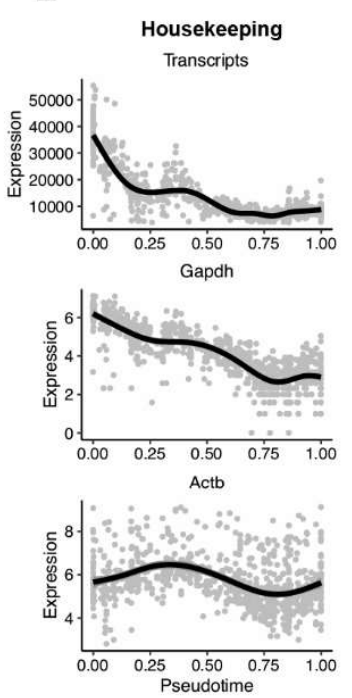

K
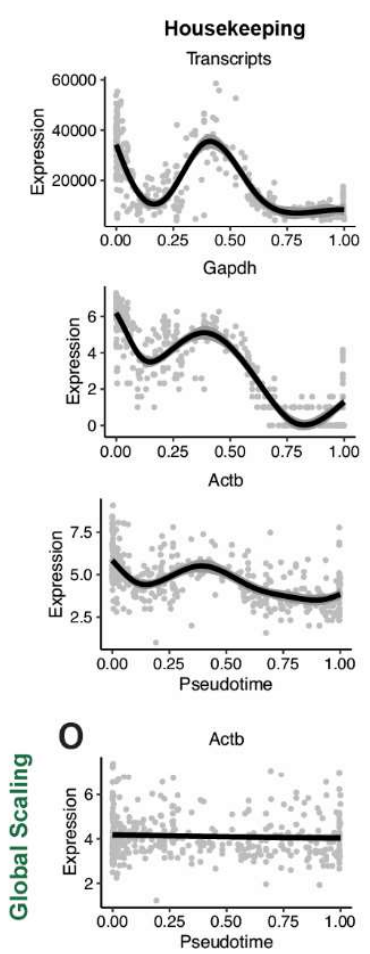

B Transcripts

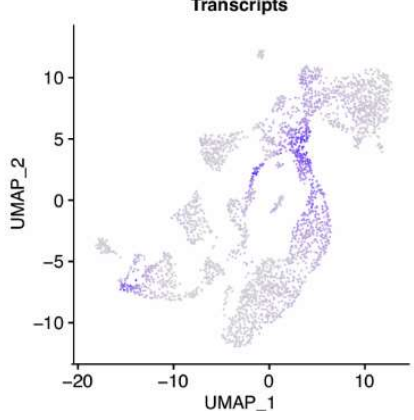

F
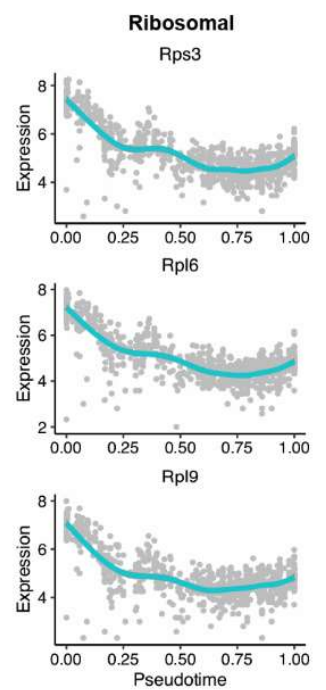

$\mathbf{L}$
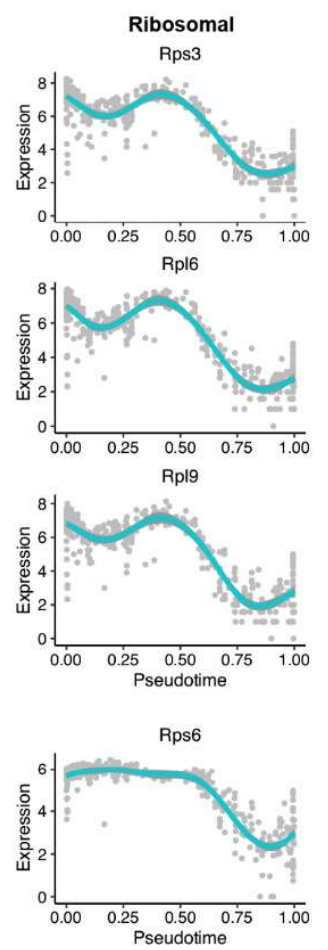

G

Chromatin Remodeling

Chd1
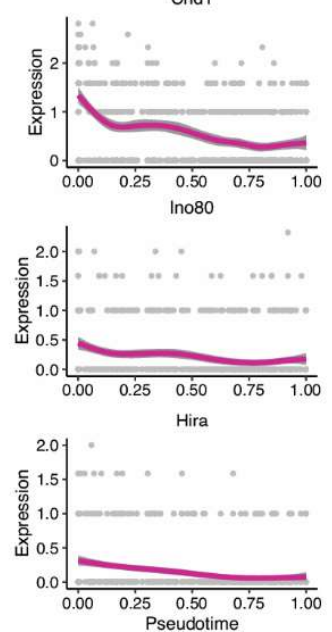

M

Chromatin Remodeling
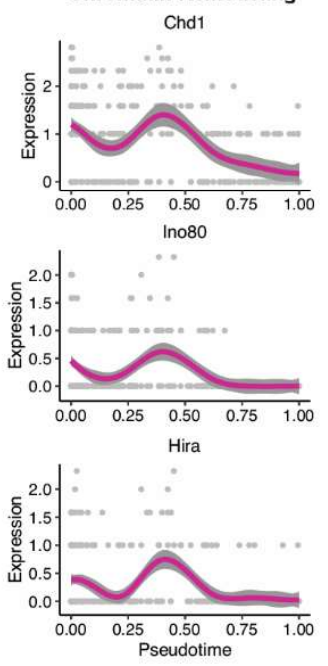

H

Monocyte Differentiation
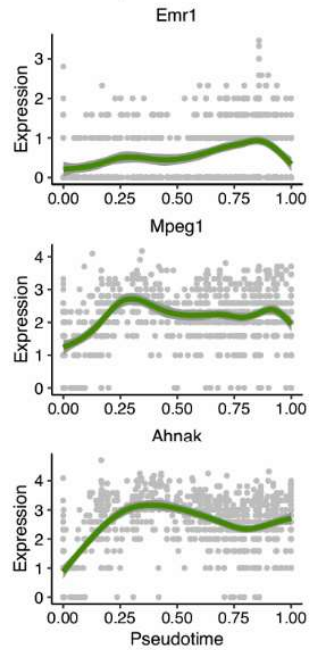

N

Erythroblast Differentiation
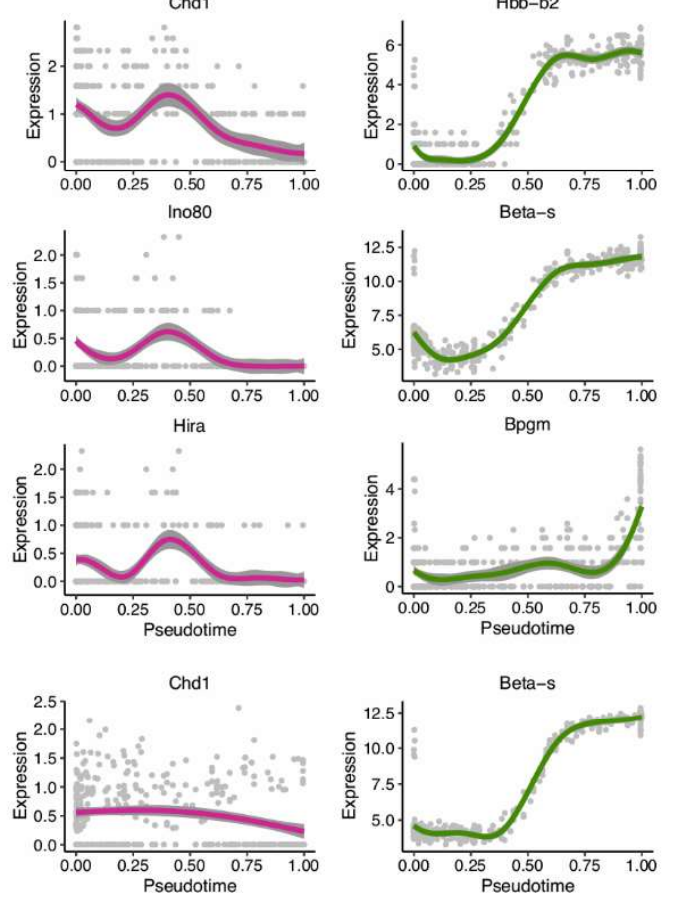